\title{
ENHANCING DOWNSTREAM PROCESSING OF ALGAL-BASED BIOFUEL USING NOVEL FUSED BACTERIA AND GREEN SOLVENTS
}

\author{
by \\ Asma Fiayaz \\ BSc., Physical Geography, York University, Toronto, Canada, 2015
}

A Thesis
presented to Ryerson University

in partial fulfilment of the

requirements for the degree of

\begin{abstract}
Master of Applied Science
in the program of
\end{abstract}

Environmental Applied Science and Management

Toronto, Ontario, Canada, 2020

(C) Asma Fiayaz, 2020 
AUTHOR'S DECLARATION FOR ELECTRONIC SUBMISSION OF A THESIS

I hereby declare that I am the sole author of this thesis. This is a true copy of the thesis, including any required final revisions, as accepted by my examiners.

I authorize Ryerson University to lend this thesis to other institutions or individuals for the purpose of scholarly research.

I further authorize Ryerson University to reproduce this thesis by photocopying or by other means, in total or in part, at the request of other institutions or individuals for the purpose of scholarly research.

I understand that my thesis may be made electronically available to the public. 


\title{
ABSTRACT \\ ENHANCING DOWNSTREAM PROCESSING OF ALGAL-BASED BIOFUEL USING NOVEL FUSED BACTERIA AND GREEN SOLVENTS
}

\author{
Asma Fiayaz \\ Master of Applied Science \\ Environmental Applied Science and Management \\ Ryerson University
}

2020

The present study investigated the utilization of algal biomass to produce bio-oil and acetone, butanol, and ethanol (ABE) products. Novel Clostridia fusants $(C$. beijernickii $+C$. thermocellum-CbCt and C. acetobutylicum + C. thermocellocum-CaCt) were developed using protoplast fusion technique and subsequently subjected to UV radiation for strain enhancement. Resultant mutated fusants showed improvement in thermal stability and higher resistance to biobutanol toxicity. Algal biomass was initially subjected to various hydrolysis treatments prior to fermentation. Combination treatment of thermal, chemical, and enzymatic resulted in maximum sugar release of $27.78 \mathrm{~g} / \mathrm{L}$. Maximum biobutanol concentration from fermentation using $C b C t$ resulted in $7.98 \mathrm{~g} / \mathrm{L}$. Fermentation using $C a C t$ produced a concentration of $7.39 \mathrm{~g} / \mathrm{L}$. Oil extraction from virgin algae investigated a green, bio-based approach using terpenes with ultrasonication and a modified, Bligh and Dyer method, separately. Combination method, ultrasonication followed by the modified Bligh and Dyer, resulted in oil yield of $46.27 \%$ (dlimonene) and $39.85 \%$ (p-cymene). Oil extraction was also produced from an algae sample following fermentation. Combined extraction method using fermentation sample resulted in oil yield of $65.04 \%$. 


\section{ACKNOWLEDGMENTS}

I would like to sincerely thank my supervisor, Dr. Yaser Dahman, from the Department of Chemical Engineering at Ryerson University for his continued support, direction, and assistance throughout my studies.

I would also like to thank the Yeates School of Graduate Studies and the Environmental Applied Science and Management program to have given the opportunity and funding support for my studies and research. I would also like to thank Andrew Laursen, the program director of the Environmental Applied Science and Management program, for his continued support and advice throughout my time at Ryerson University. Furthermore, I would also like to thank the Ryerson Staff for their assistance during my master's. A special thank you to Daniel Boothe, the Engineering Technologist of the Department of Chemical Engineering; Spencer Crook, a graduate assistant in Dr. Woolfhardt's lab at Ryerson University; Mohamed Khattab, a PhD student in the Nanocomposites and Biomaterials lab at Ryerson University, and Ahmad Chaudhary, a fellow graduate student in the Nanocomposites and Biomaterials lab at Ryerson University. I would also like to mention Shawn McFadden and Robert Denning for their support with the HPLC training and usage.

Furthermore, I would like to thank my fellow classmates in the Environmental Applied Science and Management program for their on-going support, guidance, advice and love throughout my master's.

Lastly, I would like to extend my sincerest gratitude and appreciation to my family for their unconditional love and encouragement throughout my master's. 


\section{TABLE OF CONTENTS}

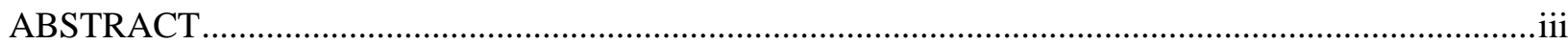

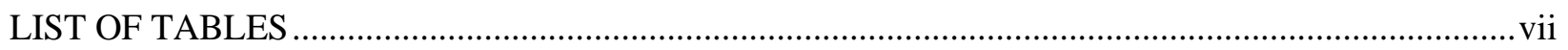

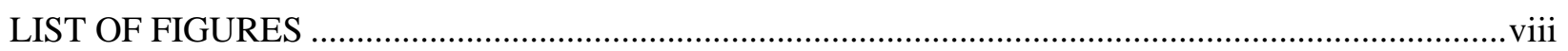

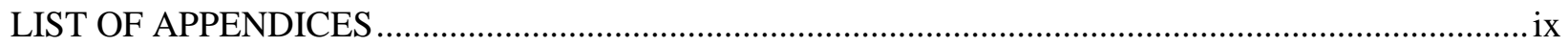

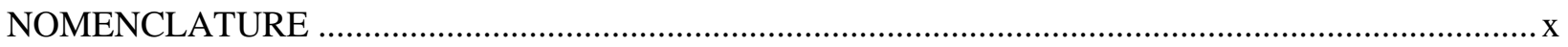

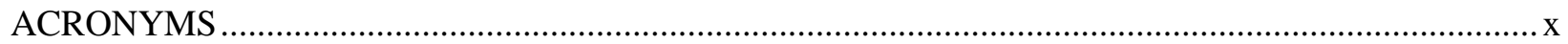

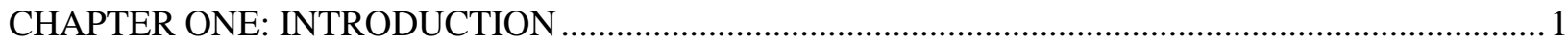

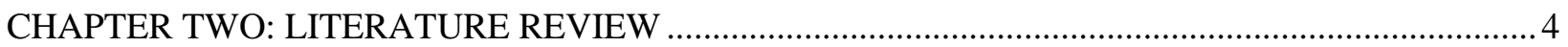

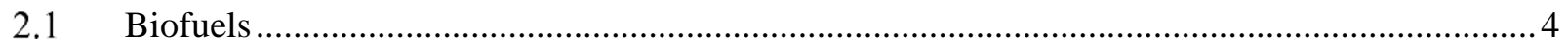

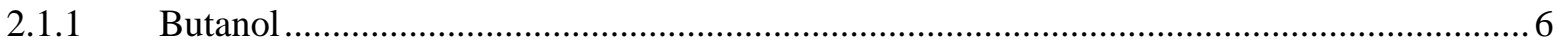

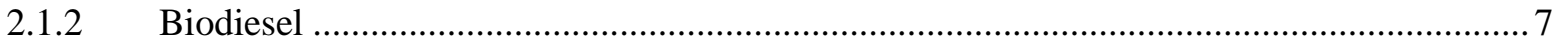

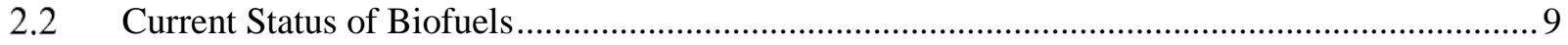

2.3 Agricultural Biomass as Feedstock ................................................................................ 12

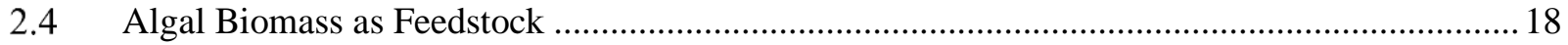

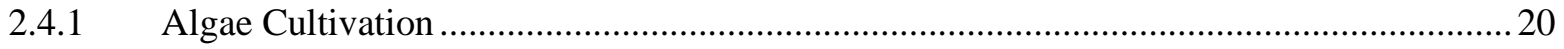

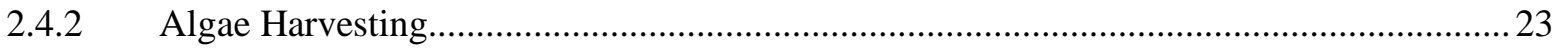

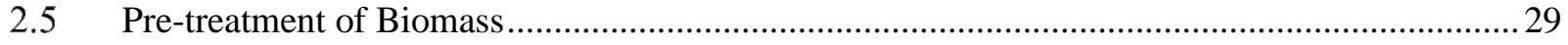

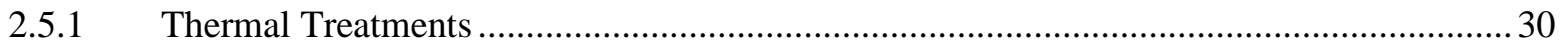

2.5.1.1 Thermal Pre-treatments of Low Temperature ................................................................... 31

2.5.1.2 Hydrothermal Pre-treatments ................................................................................... 32

2.5.1.3 Thermal Pre-treatment with Steam Explosion ………………………………………….... 33

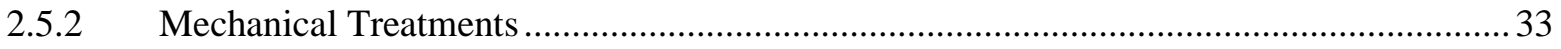

2.5.2.1 Simple Mechanical Pre-treatments ………………………………………………....... 34

2.5.2.2 Complex Mechanical Pre-treatments ........................................................................ 35

2.5.3 Chemical Treatments ........................................................................................... 37

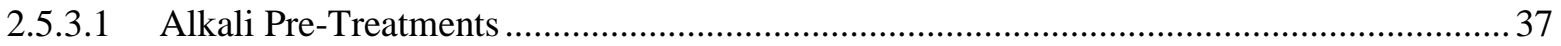

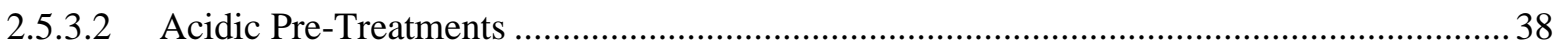

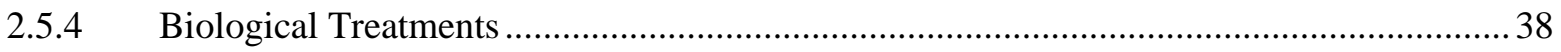

2.6 Acetone-Butanol-Ethanol (ABE) Fermentation Process …………………................................. 40

2.7 Protoplast Fusion Technology ……………………………………………………..... 42

$2.8 \quad$ Biodiesel Production Process .......................................................................................... 46

2.8.1 Algal Oil Extraction Methods ................................................................................... 47 


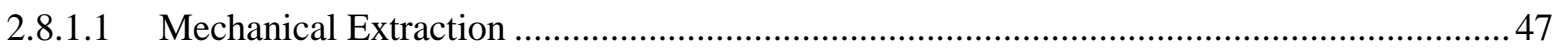

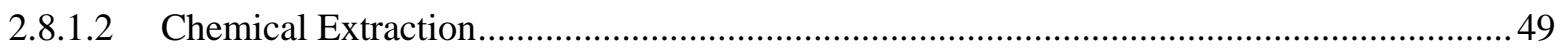

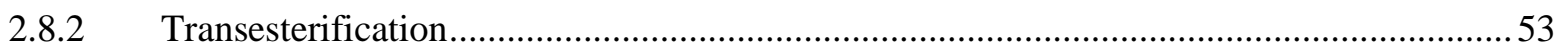

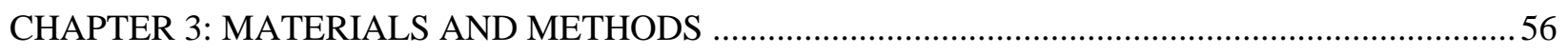

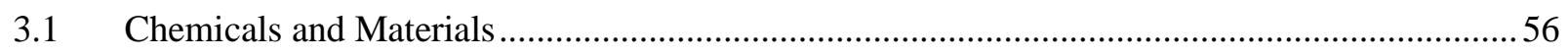

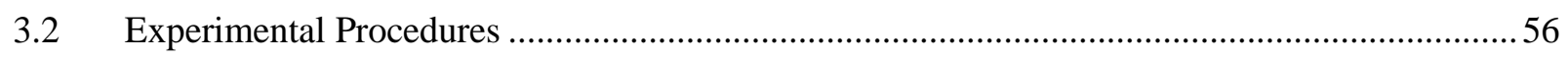

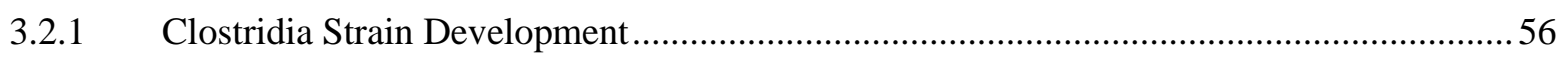

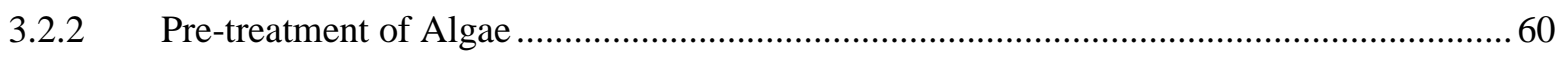

3.2.3 Biobutanol Production in Batch using Algal Biomass.................................................... 61

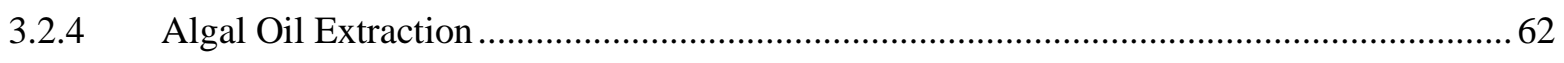

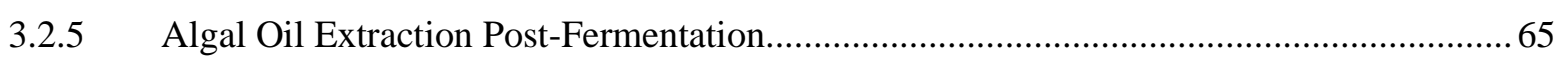

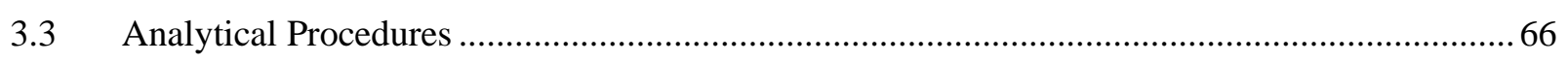

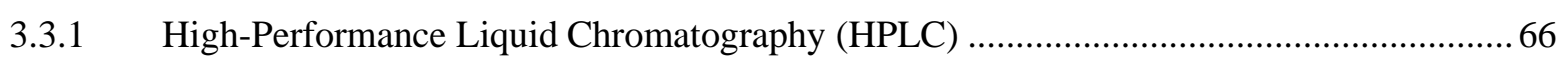

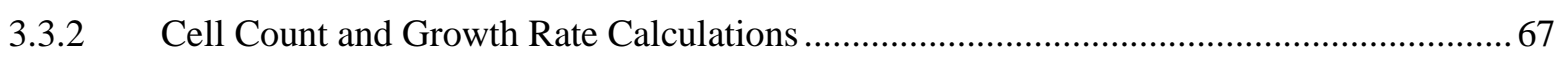

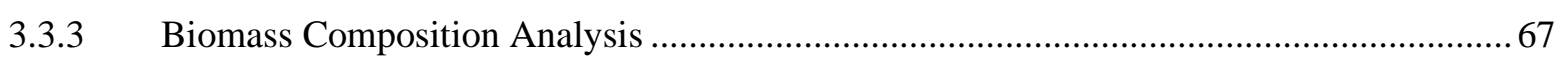

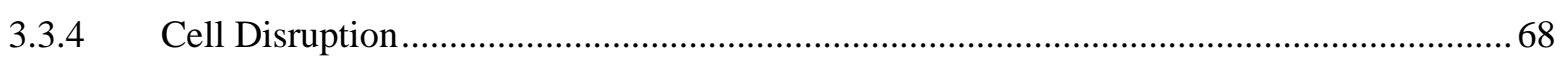

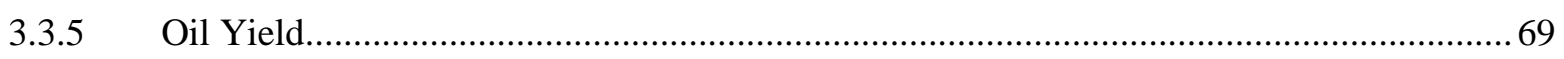

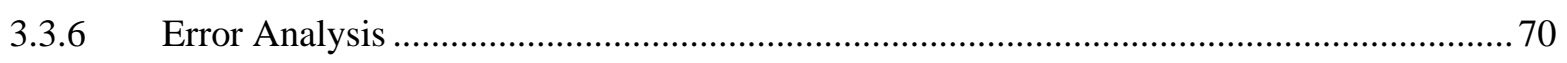

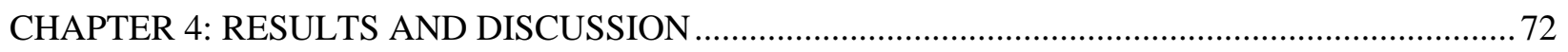

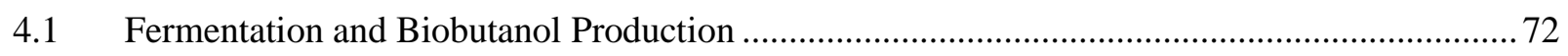

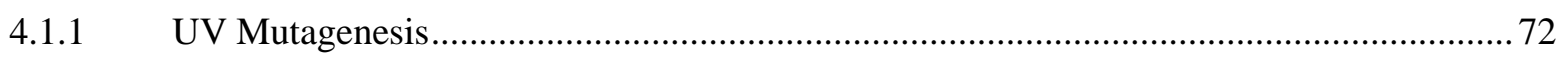

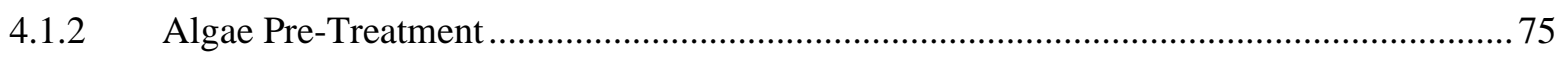

4.1.3 Simultaneous Saccharification and Fermentation and Separate Hydrolysis and

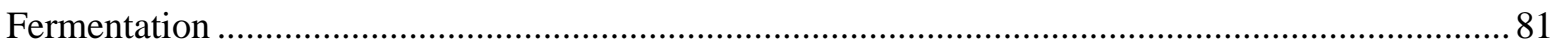

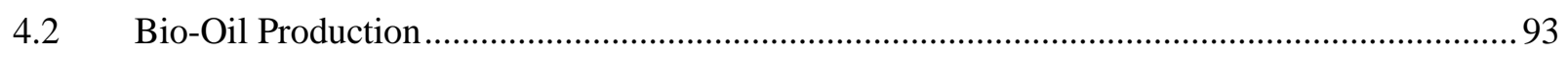

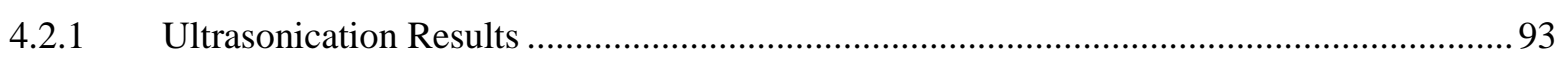

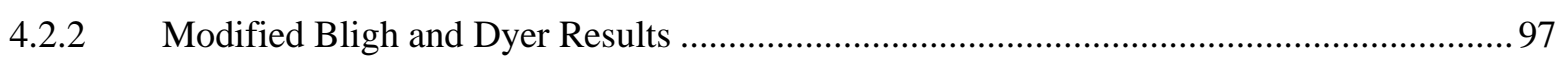

4.2.3 Combination Method: Ultrasonication + Modified Bligh and Dyer Results .................... 100

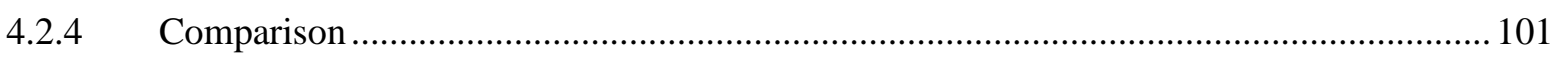

4.2.5 Algal Oil Extraction: Post-Fermentation …............................................................. 104

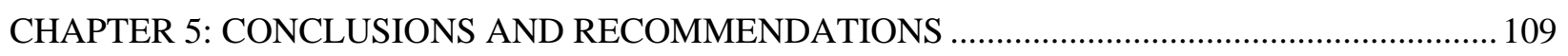

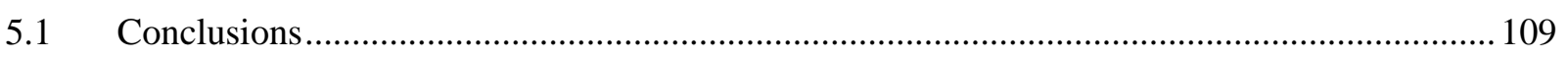

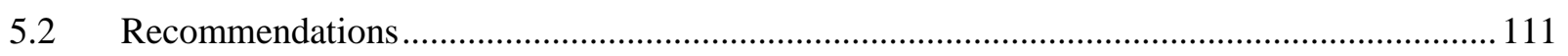

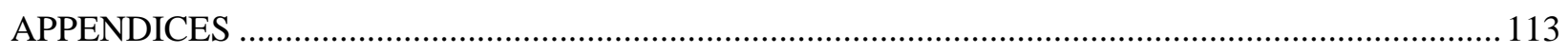

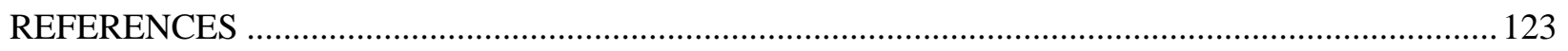




\section{LIST OF TABLES}

Table 2.1: Oil productivity of different agricultural feedstocks $\ldots \ldots \ldots \ldots \ldots \ldots \ldots \ldots \ldots \ldots \ldots \ldots \ldots \ldots$

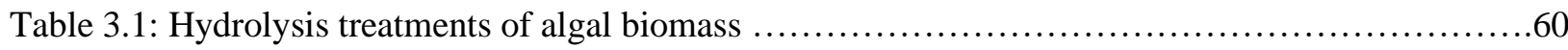

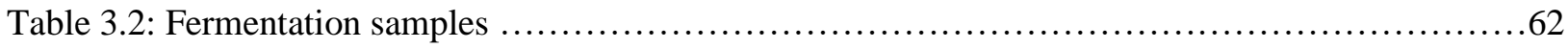

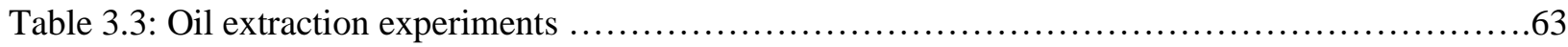

Table 4.1: Sugar Concentrations for different pre-treatment options for algal biomass .................77

Table 4.2: Lignocellulosic composition and TRS $(\mathrm{g} / \mathrm{L})$ of various agricultural residues ..................80

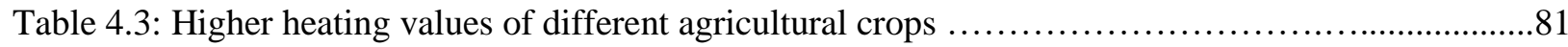

Table 4.4: Individual sugar concentrations for each fermentation experiment $\ldots \ldots \ldots \ldots \ldots \ldots \ldots \ldots \ldots . . . \ldots 2$

Table 4.5: ABE production values and sugar consumption of algae fermentation after 120 hours ........83

Table 4.6: Comparison of biobutanol concentration produced from algal biomass .....................88

Table 4.7: Comparison of biobutanol concentration produced from different agricultural sources ........92

Table 4.8: ABE and butanol yield comparison of wheat straw and algae following fermentation ........93

Table 4.9: Comparison of oil yield from different algae species ..................................97

Table 4.10: Oil yield from Modified Bligh and Dyer experiments with algal biomass using terpenes .....98

Table 4.11: Comparison of different solvents used for oil recovery for algal biomass ..................100

Table 4.12: Oil yield from combined two-step process of ultrasonication followed by Modified Bligh and Dyer with algal biomass using terpenes . ................................................. 101

Table 4.13: Cell disruption efficiency of different algae oil extraction methods .......................103

Table 4.14: Oil yield from virgin algal biomass and recovered algal biomass using combined oil

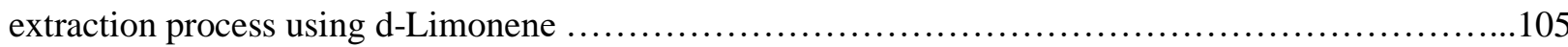




\section{LIST OF FIGURES}

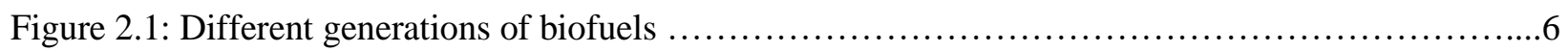

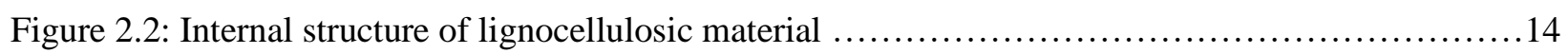

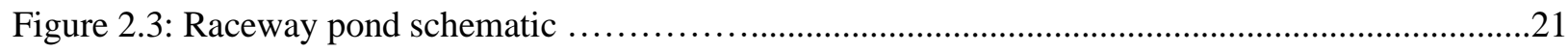

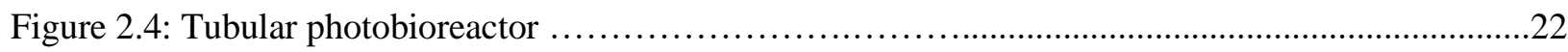

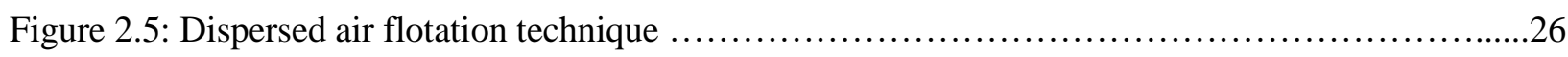

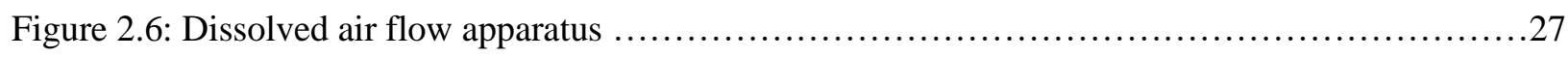

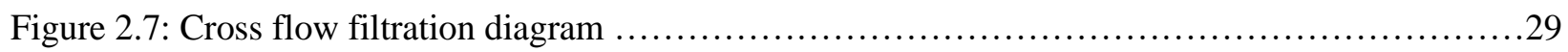

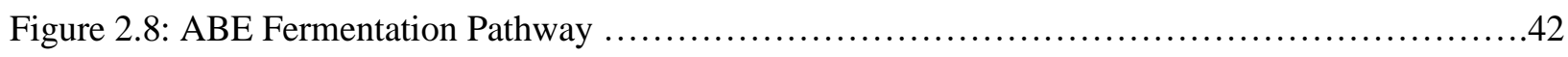

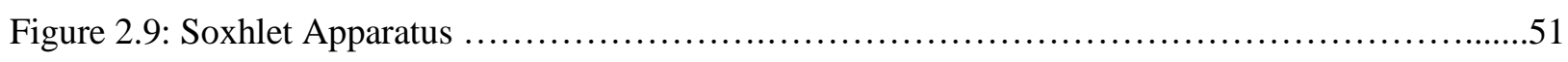

Figure 2.10:Transesterification of triglycerides with alcohol …................................5

Figure 4.1: Change in cell concentration following UV mutagenesis for fused bacteria $\mathrm{CaCt}+\mathrm{CbCt}$ in

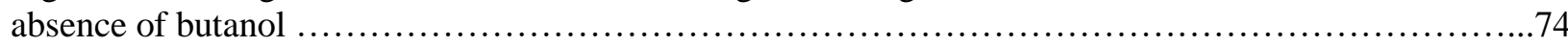

Figure 4.2: Change in cell concentration following UV mutagenesis for fused bacteria $\mathrm{CaCt}+\mathrm{CbCt}$ in

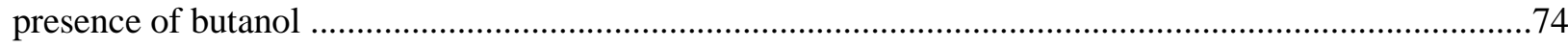

Figure 4.3: (SF) and (RF) of induced mutation from UV mutagenesis of fusant $\mathrm{CaCt}+\mathrm{CbCt} \ldots \ldots \ldots \ldots . . .75$

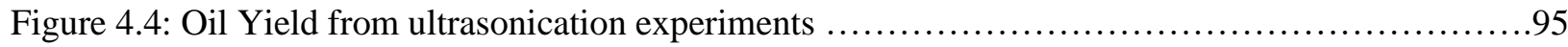

Figure 4.5: Cell disruption of different oil extraction methods ................................... 104 


\section{LIST OF APPENDICES}

Table A1: List of all chemicals used in present study .........................................113

Table B1: Cell Concentration of $\mathrm{CaCt}$ fused strain exposed to UV radiation ..........................114

Table B2: Cell Concentration of $C b C t$ fused strain exposed to UV radiation ...........................114

Figure C1: Glucose sugar HPLC calibration curve .......................................... 115

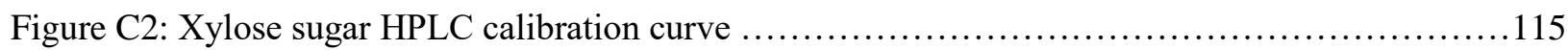

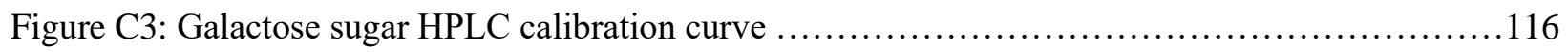

Figure C4: Arabinose sugar HPLC calibration curve ............................................116

Figure C5: Mannose sugar HPLC calibration curve ........................................ 117

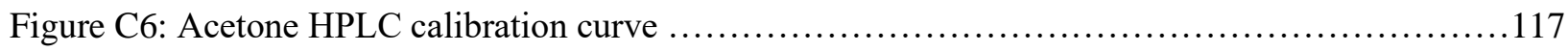

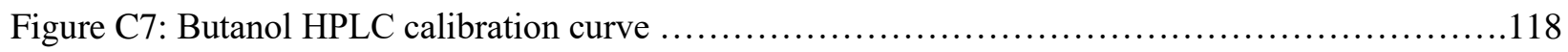

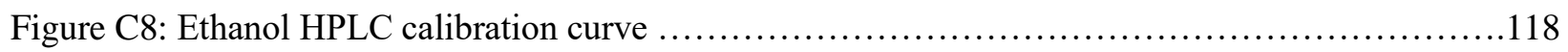

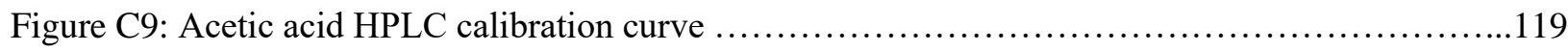

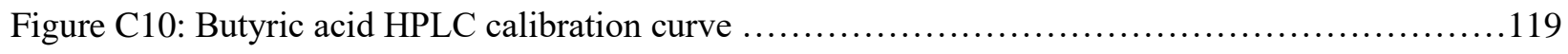

Table D1: ABE concentration for each fermentation experiment with algal biomass ..................120

Table D2: Biobutanol concentration of each fermentation experiment ........................... 120

Table D3: Total sugar concentrations for each day for each fermentation experiment .................121

Table D4: ABE yield for each fermentation experiment ..................................... 121

Table E1: Raw data for each ultrasonication oil extraction with algal biomass ......................122

Table E2: Raw data for each modified, green Bligh and Dyer oil extraction with algal biomass .........122

Table E3: Raw data for combination ultrasound and modified, green Bligh and Dyer oil extraction with

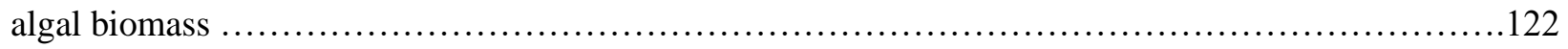

Table E4: Raw data for oil extraction post-fermentation ...................................... 122 


\section{NOMENCLATURE}

$\%$ RSD percent relative standard deviation

Ca Clostridium acetobuytilicum

$\mathrm{Cb} \quad$ Clostridium beijerinckii

$\mathrm{Ct} \quad$ Clostridium thermocellum

$\mathrm{CaCt}$ fused Clostridia strain $\mathrm{Ca}$ and $\mathrm{Ct}$

$\mathrm{CbCt} \quad$ Fused Clostridia strain $\mathrm{Cb}$ and $\mathrm{Ct}$

$\mathrm{X}$

data point

\section{ACRONYMS}

$\begin{array}{ll}\text { ABE } & \text { acetone-butanol-ethanol } \\ \text { CBM } & \text { clostridium basal medium } \\ \text { CBP } & \text { consolidated bioprocessing } \\ \text { DP } & \text { degree of polymerization } \\ \text { GHG } & \text { greenhouse gas } \\ \text { HPLC } & \text { high performance liquid chromatography } \\ \text { LUC } & \text { land use change } \\ \text { ILUC } & \text { indirect land use change } \\ \text { Mt } & \text { mega tonne } \\ \text { PEG } & \text { polyethylene glycol } \\ \text { PBR } & \text { photobioreactor } \\ \text { PPM } & \text { protoplasting medium } \\ \text { RM } & \text { regeneration medium } \\ \text { SF } & \text { surviving fraction } \\ \text { RF } & \text { relative frequency } \\ \text { SSF } & \text { simultaneous saccharification and fermentation } \\ \text { STDEV } & \text { standard deviation } \\ \text { UNFCC } & \text { United Nations Framework for Climate Change } \\ \text { UV } & \text { ultra-violet }\end{array}$




\section{CHAPTER ONE: INTRODUCTION}

Current global conditions indicate the necessity for a revitalization of the world's energy systems. Climate change, energy security, and resource depletion have become the focal point of numerous debates and subsequently, influential factors for economic, political, and social decisions worldwide. Since the Industrial Revolution humankind has heavily relied upon the combustion of fossil fuels to meet global energy demands. Fossil fuels have facilitated the production of numerous energy products and ultimately provided heating, electricity and transportation. Furthermore, fossil fuel-based energy resources have also fostered the progression of the adaptive societies seen today. However, such a reliance upon fossil fuels has come at a significant cost. Anthropogenic activities have led to the increase in $\mathrm{CO}_{2}$ emissions by approximately $25 \%$ over the past 150 years (El-Dalatony et al., 2017). Furthermore, $\mathrm{CO}_{2}$ emission are expected to rise 10\% by 2020 and 34\% by 2040 (International Energy Outlook, 2016). Raised emissions have caused substantial environmental problems including changes in weather patterns, rising sea levels and coastal flooding, biodiversity loss, and human health effects. Therefore, it is critical that alternative forms of energy be used to mediate climate change and offer carbon-reducing solutions.

Bringing sustainable, greenhouse gas (GHG) reducing and economically viable renewable sources into the energy mix has become a vital aspect of nations and technological corporations worldwide. Within the past two decades, significant research and development has been dedicated towards the field of renewable energy. Although the primary energy source for meeting the demands of the globe continue to be non-renewables, renewable energy has been a component of the energy mix throughout the history of humankind. Urgency to reduce our 
dependence upon non-renewables has resulted in the rise in biofuels. Biofuels can be defined as fuels that are derived from naturally occurring resources such as plants, crops, and agricultural residues (Demirbas, 2010). They can be utilized for a variety of applications including heating, electricity and producing gas-like fuels. Liquid fuels derived from naturally occurring sources are utilized as alternatives in the transportation industry (Bulkowska et al., 2016). Biofuels can be categorized into two main groups primary and secondary. Primary biofuels are comprised of biomass that can be directly used without any processing or conversion (Demirbas, 2010 and Bulkowska et al., 2016). These include wood, wood chips, and pellets which have been used by humankind before any form of technological innovation for electricity production, heating, and cooking. Secondary biofuels are different because they are developed by processing biomass into different biologically based products such as liquid fuels (Demirbas, 2010 and Bulkowska et al., 2016). Secondary biofuels can be further categorized into first, second, and third generation based upon their feedstock (Demirbas, 2010 and Bulkowska et al., 2016).

Biofuels have been a prominent part of the energy industry for many years. However, recent assessment can be indicative of the issue that first and second-generation biofuels are failing to replace non-renewables. Furthermore, they have been criticized for the minimal capacity to reduce carbon emissions and their associated high production costs. Over the years, viable alternatives have been researched to improve the capacity and reputation of biofuels. Recently, the scientific community has shifted interests towards utilizing a third-generation feedstock of algae as potential biofuel feedstocks (Gouevia, 2011; State of Technology ReviewAlgae Bioenergy, 2017). When compared to traditional feedstock choice, algae offer a wide array advantageous characteristics and properties that provide opportunity for improving biofuel output into the energy industry. Due to its recent emergence into the scientific community, 
bringing algal-based biofuel to the commercial scale is still a challenging endeavour (Zhang et al., 2016; Adeniyi, 2018). Improvements on many fronts must be achieved prior to the adaption of this third-generation biofuel.

The present study focuses on investigating the downstream processing of algal-based biofuel production by using novel fused Clostridia strains and bio-based terpenes. Protoplast fusion technology was applied to develop novel fused bacteria strains of Clostridia sp. The fusants were exposed to UV radiation to produce desirable mutants used during the fermentation process to produce biobutanol using algal biomass. The algal biomass was subjected to various hydrolysis pre-treatments prior to the fermentation process. Additionally, this study also investigated the use of ultrasonication and the green, modified Bligh and Dyer method using terpenes to extract oil from algal biomass. Oil extraction was achieved from virgin, untreated algal biomass and algal biomass recovered following the fermentation process. In the present study, fermentation was deployed prior to oil extraction because it has been recognized that fermentation processes do not affect the fatty acid profile and quality of the lipids (Amit et al., 2011 and Ruthu et al., 2014). Algal biomass showing the highest biobutanol production from fermentation was then examined for oil extraction process. Furthermore, the extraction conditions producing the highest oil extraction yield, when using virgin algal biomass, were applied to the recovered algal biomass. 


\section{CHAPTER TWO: \\ LITERATURE REVIEW}

\subsection{Biofuels}

Biofuels can be described as an alternative fuel that is developed from biological, natural, and renewable sources (Demirbas, 2009). Production of biofuels relies upon sources such as plants, crops, and agricultural residue. As discussed, biofuels are classified into two groups: primary and secondary (Figure 2.1). Primary biofuels have been described as biomass that can be directly used without processing and they include wood, wood chips and pellets (Demirbas, 2010 and Bulkowska et al., 2016). Furthermore, primary biofuels have been utilized for many years to fulfill heating, cooking and electrical demands (Demirbas, 2010 and Bulkowska et al., 2016). On the contrary, secondary biofuels are those that are produced through the processing of biomass. Secondary biofuels can be further categorized into first, second, and third generation based upon their feedstock (Demirbas, 2010 and Bulkowska et al., 2016).

First-generation biofuels are produced from food crops such as corn and wheat (Singh et al., 2017). Second-generation biofuels are produced from non-food crops including the waste from food-crops, agricultural residue, wood chips and waste cooking oil (Singh et al., 2017). Lastly, third-generation biofuels are those that are considered relatively new in the field and they primarily include algae as well as fast growing trees (Singh et al., 2017). Biofuels have been regarded as a sustainable alternative as they have the capacity to reduce GHG emissions up to 90\%; depending on the biomass (Demirbas, 2009). Additionally, biofuels also have the added benefit of being blended with conventional fuels or being used entirely on their own (Demirbas, 2009). 
The concept of utilizing biologically and naturally occurring products, found in nature, began in the early 1700s when lamps relied upon vegetable oils, whale oil, and lard oil (Singh et al, 2017). Following this, ethanol blended with turpentine was utilized for illumination. Subsequently, ethanol began to be of interest within various nations worldwide including Germany, United States, and the United Kingdom. Research and development initiatives in Germany with respect to ethanol-fueled trucks and automobiles began in 1899 and subsequently, further promotion of ethanol-fuel household appliances in 1902 (Singh et al, 2017).

Consequently, this spread a global interest in biofuels, specific to that of ethanol, in other nations including the United States, France, Italy, and Spain. Due to the scarcity of oil resources, the interest in biofuels began to experience growth during the early 1900s particularly in the United Kingdom and France (Singh et al, 2017). During the 1920s and 1930s, the United States began to incorporate biologically based products, such as ethanol, in their transportation industry. Henry Ford provided the necessary momentum to facilitate the promotion of chemurgy which ultimately focused on crop utilization to produce industrial materials (Singh et al, 2017). With this concept, by $194377 \%$ of synthetic rubber produces in the United States incorporated ethanol (Singh et al, 2017).

On-going research and development initiatives have made it possible to not only bring ethanol fuel to a larger scale but to also explore various other alternatives. As such, alternatives such as biodiesel have also experienced significant growth and utilization. For instance, between the years of 2008 and 2011, the United States' production of ethanol coupled with biodiesel increased more than 40\% (Singh et al, 2017). Additionally, the strong theoretical knowledge to support the growth and progression of the biofuels industry provided the set-up and implementation of second-generation biofuels as the first cellulosic ethanol commercial scale 
plant was fully operational in 2013 (Singh et al, 2017). Through successive scientific research and industrial practices, a wide variety of biologically based products have come into play. These include bioethanol, biodiesel, biogas, and biobutanol.

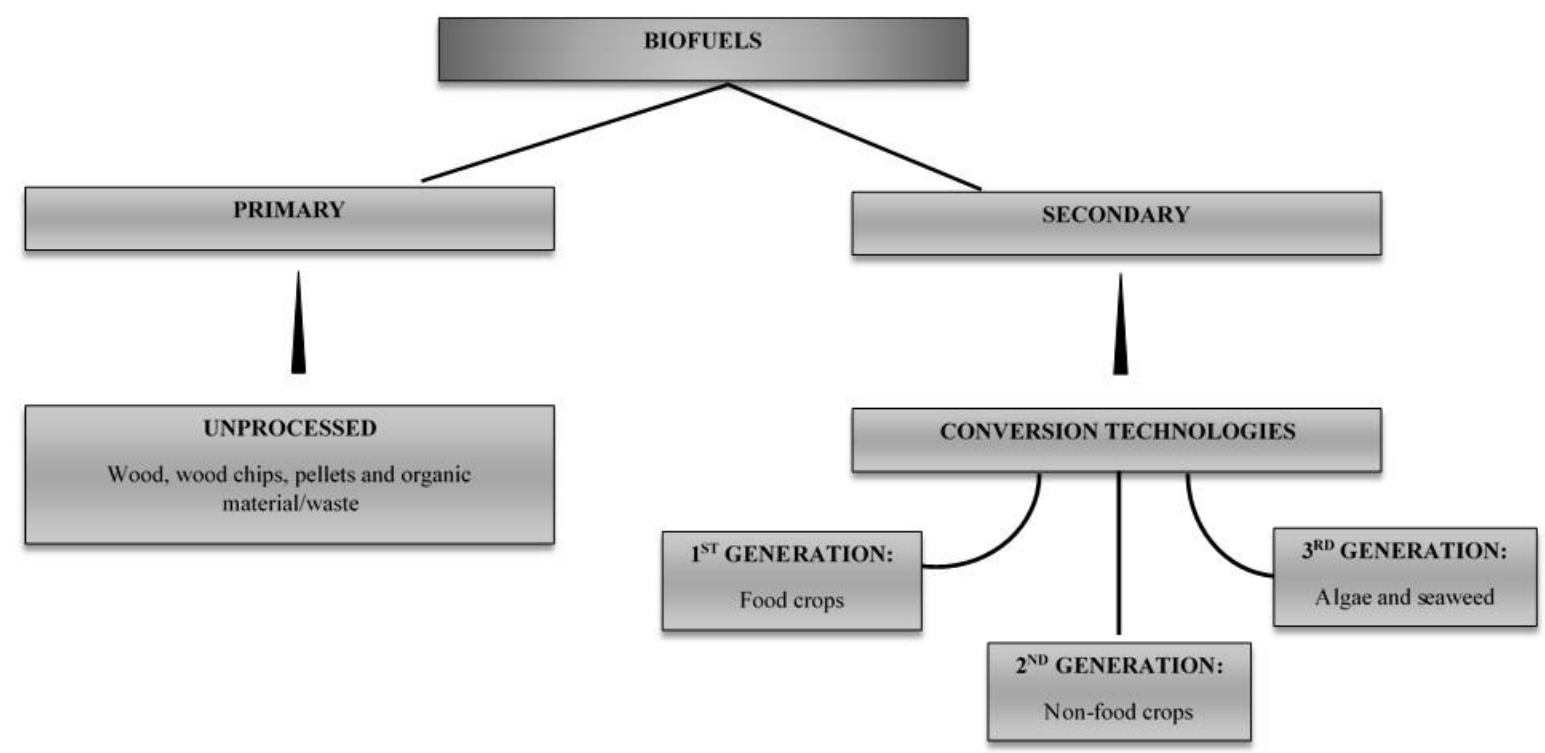

Figure 2.1 Different generations of biofuels (Adopted from Singh et al., 2017 and Demirbas, 2009)

\subsubsection{Butanol}

Butanol is an alcohol comprised of a 4-carbon structure with the chemical formula $\mathrm{C}_{4} \mathrm{H}_{10} \mathrm{O}$ (Bulkwoska et al., 2016). It has been primarily used as a solvent found in paints and can also be utilized as a fuel. Despite its recent emergence as an alternative fuel and a means to mitigate climate change, butanol possesses a long history dating back to the late $1800 \mathrm{~s}$ (Demirbas, 2009; Singh et al., 2017; Yeong et al., 2018). Initial production of biologically based butanol, referred to as biobutanol, began in the late 1800s and early 1900s (Gabriel, 1928; Singh et al., 2017). Louis Pasteur discovered the acetone-butanol-ethanol (ABE) pathway in which butanol is produced (Gabriel, 1928). Significant reliance upon biobutanol commenced during alcoholic prohibition in the United States as there was an amyl alcohol scarcity for the paint 
industry (Singh et al., 2017). Biobutanol served as a replacement for the amyl alcohol and subsequently numerous $\mathrm{ABE}$ fermentation plants were created to provide a biobutanol supply to the paint industry (Durre, 2005). Although having a strong reliance upon biobutanol until 1950, the paint industry then turned to a cheaper alternative; butanol produced from chemical synthetic processes relying on petrochemical feedstocks (Durre, 2005). Although the interest and subsequent production of biobutanol experienced a lag following competitive and cheaper alternatives, it is now of greater interest and high priority due to submerging issues of climate change and energy crises.

When compared with ethanol, butanol has an energy content of $29.2 \mathrm{MJ} / \mathrm{L}$ which is $30 \%$ higher than that of ethanol, rated at 21.3 MJ/L (Nanda et al., 2014). Furthermore, the energy content of butanol is also within a much closer range than that of gasoline; rated at $32.5 \mathrm{MJ} / \mathrm{L}$ (Nanda et al., 2014). With respect to blending capacity, butanol can be blended up to $100 \%$ or can be used in pure form (Nanda et al., 2014). Moreover, butanol also possesses low vapor pressure, low volatility, and low flammability (Nanda et al., 2014). Its hydroscopic nature also proves to be an asset because it makes a desirable fuel to be transported through pipelines similar to gasoline (Nanda et al., 2014).

\subsubsection{Biodiesel}

In addition to bio-alcoholic fuels such as ethanol and butanol, biodiesel has generated elevated levels of interest throughout the globe. It has become one of the most attractive forms of alternative and renewable biofuels (Issariyakul and Dalai, 2014). Furthermore, a 5.54 fossil energy ratio was recently reported for biodiesel which indicates that one unit of fossil energy input is required to produce 5.54 units of biodiesel energy output. This fossil energy ratio is considered to surpass other fuels as well and is also expected to increase in the coming years due 
to increase crop yield, the adoption of energy-saving farm practices, and the on-going development of energy efficient technologies. When compared to conventional, petroleum-based diesel, biodiesel is a highly attractive alternative as it offers lower exhaust emissions, is biodegradable, non-toxic, renewable and contains no sulfur.

Biodiesel is mono alkyl esters of long chain fatty acids that can be produced from acylglycerol usually in the form of triglycerides through the process of transesterification (Demirbas and Demirbas, 2011; Knothe and Razon, 2017). Through this process, the parent oil or fat is converted to biodiesel with a viscosity close to that of petroleum-based diesel. This chemical conversion process of oil is described below in the following reaction:

Triglyceride +3 methanol $\leftrightarrow$ Glycerine +3 methyl ester (Biodiesel)

Biodiesel can be produced from a variety of lipid feedstocks including vegetable oils, and animal fats. Most common lipid feedstock choices have been vegetable oils such as rapeseed, palm, soybean, and coconut (Knothe and Razon, 2017). However, recent scientific interest has been evaluating algae as lipid feedstock choices for biodiesel production. Due to the many advantages of algal biomass, as discussed in this study, utilizing algae as a lipid feedstock choice has begun to spark great interest for the biofuels market with respect to biodiesel production.

Algae store lipids in the form of triaglycerides (TAGs) which can be transestrified to produce biodiesel. Many species of algae accumulate lipid quantities up to $60 \%$ of their dry biomass. When compared with other lipid feedstocks, algal biomass has the potential to produce oil yields up to $100,000,000 \mathrm{~L} /$ hectare as depicted in the table below. However, this value can vary within different algal species depending on culture and growth conditions. Optimal conditions can be created through the fluctuations of nutrients and most importantly, the 
availability of $\mathrm{CO}_{2}$ (Demirbas and Demirbas, 2011). Another critical characteristic of algae when examining its lipid feedstock potential is the energy density of the perspective biodiesel. Petroleum diesel has an energy density of $42.7 \mathrm{Mj} / \mathrm{kg}$. Commonly used lipid feedstocks such as rapeseed or soybean have energy densities of $39.5 \mathrm{Mj} / \mathrm{kg}$ whereas algal produced biodiesel has an energy density of $41 \mathrm{Mj} / \mathrm{kg}$ (Demirbas and Demirbas, 2011; Demirbas, 2007; Rakopolous et al., 2006; Xu et al., 2006). In addition to the higher lipid values and energy density of biodiesel produced from algal oil, algal biomass possesses other advantages such as high productivity and rapid growth rate which make it an enticing and superior alternative.

Table 2.1 Oil productivity of different agricultural feedstocks (Demirbas and Demirbas, 2011)

\begin{tabular}{|l|l|}
\hline Crop & Oil in litres per hectare \\
\hline Algae & $100,000,000$ \\
\hline Castor & 1413 \\
\hline Coconut & 2689 \\
\hline Palm & 5950 \\
\hline Safflower & 779 \\
\hline Soy & 446 \\
\hline Sunflower & 952 \\
\hline
\end{tabular}

\subsection{Current Status of Biofuels}

Despite the theoretical evidence to suggest that biofuels have the potential to be a promising tool for climate change mitigation, current consensus and evidence state that biofuels have fallen short. Recent assessments of first- and second-generation biofuels have indicated that they have made minute reductions in GHG emissions, placed a significant financial burden upon 
governments, and have, despite their initial promise, brought on a series of environmental impacts. The barriers associated with the successful development of biofuels can be linked to technology, policy, and economics which are all subsequently tied to governments (Demirbas and Demirbas, 2011). Consequently, governments play an integral role in the continued research and development as well as the progression of the biofuel industry. Successful deployment and integration of a biofuel industry in the energy mix requires vast amounts of government subsidies (Eggert and Greaker, 2014). In Canada, initial emergence of biofuels into the market brought on various amounts of government subsidies to assist developers. However, it was anticipated that biofuels would only contribute to $1 \%$ of the previous climate targets designated under the Kyoto Protocol (Roy and Klein, 2012). To increase the contribution of biofuels for GHG emission reductions, the Canadian federal government introduced the Renewable Fuel Standard which require fuel producers and importers to blend an average renewable fuel content of $5 \%$ for gasoline and 2\% for diesel (Eggert and Greaker, 2014). With this initiative, along with government subsidies, the biofuel industry took off. However, it is also important to note that between 2010 and 2015, biofuel consumption corresponded with GHG reductions equivalent to approximately $3 \mathrm{Mt}$ (Canada's EcoFicscal Commission,2016). This attributed to only $0.4 \%$ of the total GHG emission reductions in Canada (Canada's EcoFicscal Commission, 2016). In addition to the insignificant reduction of emissions, the industry has also been recognized as a financial burden for the government as well as tax-payers (Canada's EcoFicscal Commission 2016). The associated cost of reducing emissions through biofuels is approximately $\$ 180-\$ 185$ per tonne with ethanol policies whereas fewer conservative values are indicated within the range of \$234-\$284 per tonne (Canada’s EcoFicscal Commission, 2016). 
First and second-generation biofuels have also raised significant concerns regarding their environmental impacts. Primary biofuel production in Canada is reliant upon wheat and corn and is recognized as a first-generation biofuel industry (Roy and Klein, 2012). Other nations relying upon biofuels to meet their climate targets, have shifted from first-generation to secondgeneration biofuels due to the significant drawbacks. One of the most controversial issues associated with first-generation biofuels is that they require food crops to produce liquid fuels which has sparked the food vs. fuel debate (Demirbas, 2009 and Gouevia, 2011However, second-generation biofuels have also been criticized for their environmental burdens. Both first and second-generation biofuels require an abundance of land for growth and are accompanied with burdens upon agricultural resources and water (Roy and Klein, 2012; Eggert and Greaker,2014). Several studies have indicated the impacts of land-use changes (LUC) and indirect land use changes (ILUC) upon carbon emissions (Plevin et al., 2010; Czyrnek-Deletre et al., 2016). The emissions avoided through the consumption of biofuels may be offset by the emissions from both LUC and ILUC. LUC are associated with changes that arise when, for instance, a type of land is converted for a bioenergy plantation (Ahlgren and Di Lucia, 2014). ILUC are associated with changes that arise when land use causes a displacement in food or feed producers which ultimately requires a re-establishment of natural ecosystems (Ahlgren and Di Lucia, 2014). Yasarer et al. (2016) also examined the implications of specific crop growth for biofuels on the water quality in the Kansas watershed in the United States. Through a soil and water assessment tool, it was indicated that the expansion of corn and grain sorghum, which are the prime crops used for ethanol production, resulted in high sediment and nutrient loading into the Kansas watershed thus negatively effecting water quality (Yasarer et al., 2016). 


\subsection{Agricultural Biomass as Feedstock}

Biofuel production is reliant upon a variety of materials that are renewable and naturally occurring in the environment. Such materials are referred to as biomass and they are described as plant-based material. According to the United Nations Framework Convention on Climate Change (UNFCC), biomass is recognized as non-fossilized and biodegradable organic substance that stems from plants as well as animals and micro-organisms (UNFCC). Further analysis of biomass has rendered two distinct categories: virgin and waste. Virgin biomass referrers to biomass incorporated through terrestrial and aquatic means such as forest biomass, grasses, energy crops, and algae (Basu, 2010). On the contrary waste biomass refers to biomass acquired through municipal, agricultural, forestry, and industrial applications (Basu, 2010). Biomass is considered to be the oldest source for fuel energy. Heating and energy demand until the $19^{\text {th }}$ century were primarily fulfilled through biomass in the form of firewood and charcoal (Yokoyama, 2008). With technological changes and subsequent advancements, fossil-based alternatives such as coal and oil, became the alterative choice. However, within the past decade, significant attention has been placed on the expansion of biomass production for the purpose of fuel energy. Rampant concerns of climate change and energy security issues have led to the prospect of utilizing biomass for the production of transport fuels.

Due to the diverse availability of biomass, a variety of different biofuels can be rendered possible. Through a series of biomass conversion pathways, both virgin and waste biomass can yield different forms of biofuels; ranging from biodiesel, bioethanol and biogas (Bułkowska et al, 2016). As stated, first-generation biofuels are derived from conventional technologies as they contain simple constituents that can be broken down easily. On the contrary, second and thirdgeneration biofuels are derived from complex materials which require as specific pre-treatment 
to break down the biologically available compounds. Biomass utilized to produce second and third-generation biofuels stem from lignocellulosic material. Lignocellulosic material is the most abundant renewable biomass on Earth and plays an increasingly integral role in current, global biofuel production (Araujo et al, 2017). Second and third-generation biofuels can be distinguished from their predecessor as they utilize biomass containing lignocellulosic material which is comprised of three main constituents; cellulose, hemicellulose, and lignin. These three components are strongly bound together as a result of non-covalent forces and covalent crosslinks which ultimately lead to its complex structure as depicted in Figure 2.3 (Bułkowska et al, 2016). As such, bioavailability and subsequent production of biofuels is highly dependent upon the fragmentation and disruption of the lignocellulosic composition. Converting lignocellulose material to biofuels requires enough decomposition because of its recalcitrance to biodegradability (Bulkwoska et al., 2016). The cell wall and its components are the prime reason for the biodegradability resistance experienced by lignocellulosic materials. It is an important component of biomass and possesses a rigid, compact structure. Majority of terrestrial plants contain cell walls with cellulose, hemicellulose, and lignin as well as structural components such as protein and minerals (Vogel et al., 2008). Cellulose forms as a hard outer later and is surrounded by other polymers functioning as a matrix which are hemicelluloses. Furthermore, the lignin encrusts the cellulose and hemicelluloses. Non-structural components of the cell wall include sugars, pectin, proteins and minerals. Different elements and characteristics of the cell wall contribute to the recalcitrance of lignocellulosic materials. Specific characteristics of cellulose, hemicellulose, and lignin directly correlate with chemical and biological degradation. 


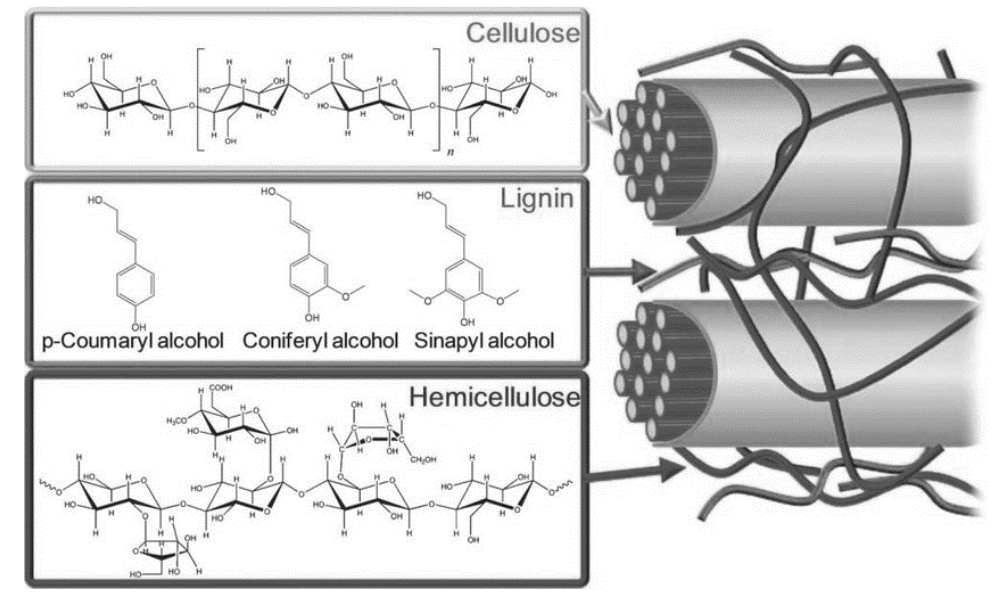

Figure 2.2 Internal structure of lignocellulosic material (Alonso et al., 2012)

Cellulose is the main constituent of lignocellulosic biomass and the most abundant polysaccharide on Earth (Kirk-Othmer, 2011; Mood et al, 2013). Its physical composition is representative of a glucose polymer linked by B-1,4 glycosidic bonds and its basic building block is cellobiose (Harmsen et al, 2010). A critical property of cellulose is the degree of polymerization which is essentially the number of glucose units that comprise one polymer molecule (Gupta and Tuohy, 2011; Bulkowska et al, 2016). The range of this degree is most commonly within 800-10,000 units however the degree can extend upwards of 17,000 units in wood pulp (Gupta and Tuohy, 2011). In simple terms, cellulose contains carbohydrate chains which can be appropriately broken for sugar conversion to develop alcoholic fuel. In scientific terms, such chains are developed as a result of B-1,4 glucosidic bonds (Gupta and Tuohy, 2011). Upon the exterior of such bonds are hydroxides which subsequently enable strong intermolecular hydrogen bonds between hydroxyl groups of adjacent molecules situated upon parallel chains (Faulon et al, 1994). The structure of cellulose can either be crystalline or non-crystalline. The crystalline structure arises due to the fusion of several polymer chains which in turn builds 
microfibers (Gupta and Tuohy, 2011). Properties of hydrophilicity, chirality, degradability, and the broad chemical variability that arises because of the high donor reactivity of - $\mathrm{OH}$ groups are directly linked to the molecular structure of cellulose. Extensive hydrogen bounds, which arise from the presence of hydroxyl groups attached to each glucosyl ring, allow for a partially crystalline fiber structure and morphology (Klemm et al, 2005). The crystalline fibers can either be amorphous or crystalline with the following organizations (1) amorphous shell surrounding a crystalline core (2) crystalline and amorphous parts alternating longitudinally of the fibril or (3) a combination of both (Salmen and Bergstorm, 2009). The crystallinity of cellulose plays an important role in biofuel production and the hydrolyzing of feedstock. Furthermore, this specific property directly corresponds to the recalcitrance nature of lignocellulosic material. Crystallinity impacts the physical, mechanical, and chemical properties of glucose (Bulkwoska et al, 2016). For instance, increasing crystallinity subsequently increases tensile strength, dimensional stability, and density. However, the increasing of crystallinity decreases chemical reactivity and swelling. Therefore, the region of the cellulose which is crystalline becomes more resistant to enzymatic, chemical, and biological degradation. The degree of polymerization (DP) also plays a critical role in the recalcitrance of lignocellulosic material. The DP experiences a wide range of 100 to 100,000 (Zhang and Lynd, 2004). For instance, agricultural residues such as wheat straw have lower DP; approximately 1000 (Hallac and Ragauskas, 2011). DP corelates with the solubility of cellulose in such a manner that a higher DP corresponds with a lower solubility. The reason for this is because a higher DP results in the presence of intermolecular hydrogen bonds which are strongly bound together. Another factor influencing the recalcitrant nature of lignocellulosic material is the accessible surface area. Physical contact between enzymes and the substrate is an important element necessary for the hydrolysis of cellulose. The accessibility of 
the cellulose by enzymes such as cellulase is limited by the following two factors (1) the structure of cellulose microfibrils, that happen to be in sizes within orders of nanometer and (2) the anatomical structure of the plant cell wall (Yang et al., 2011).

Hemicellulose is recognized as a homopolymer and is a form of a heterogenous polysaccharide (Amidon et al, 2011). This polysaccharide is the linking material between the cellulose and lignin. Additionally, what sets hemicellulose apart from cellulose is that it consists of monosaccharides. Physical components of hemicellulose incorporate short, highly branched polymers of six-carbon sugars (glucose, mannose, and galactose) and five-carbon sugars (xylose, arabinose, and glucose) (Bułkowska et al, 2016). Such short branches are also amorphous which ultimately provide hemicellulose with the capacity to be partially soluble in water (Gupta and Tuohy, 2011). Moreover, physical characteristics of hemicellulose also indicate that its backbone is comprised of a homopolymer or a heteropolymer; generally consisting of single sugars or a mixture of different sugars; respectively (Gupta and Tuohy, 2011). Xylose is the most important sugar found in hemicellulose. Amongst hardwood terrestrial plants, xylose is the main constituent of the backbone chain which provides the lack of crystalline structure (Kirk-Othmer, 2011). Hemicelluloses can also act a barrier for the accessibility and subsequent hydrolysis of cellulose. Within the plant cell wall, cellulose is connected by hemicellulosic polysaccharides such as xyloglucan or arabinoxylan which contribute to a cellulose-hemicellulose network. It has been analyzed that this network can be manifested in two different forms. In the first model, the xyloglucans or arabinoxylans coat themselves upon the cellulose microfibrils and span the 20-40 nm gap between each microfibril subsequently acting as load-bearing tethers. The second model illustrates that there is no link between the cellulose microfibrils because the xyloglucans or arabinoxylans are trapped in between. The hemicellulose can be found tightly bound to the 
microfibrils, sheathed in tight layer and are embedded within a pectin matrix. Consequently, the hemicelluloses form a significant physical barrier to the cellulose. It has been assessed that removing at least $50 \%$ of the hemicelluloses is critical to the enzymatic digestibility of cellulose (Sierra et al., 2008). The recalcitrant nature of lignocellulosic material is also a product of acetyl groups which happen to be on the backbones or branches of hemicelluloses (Gille and Pauly, 2012). Acetyl groups have been known to play an important role in the resistance mechanism of the plant cell wall to enzymatic hydrolysis of cellulose. As the hemicellulose backbones become deacetylated, the cellulose becomes 5-7 times more digestible (Grohmann et al, 1999).

Lignin can be simply described as the binding material which keeps cellulose and hemicellulose in a compressed structure (Agbor et al, 2011). Furthermore, upon examination of its physical structure it is quite different than cellulose and hemicellulose. It is a threedimensional polyphenolic network comprised of dimethoxylated, monomethoxylated, and nonmethoxylated phenylpropanoid units (Gupta and Tuohy, 2011). Lignin is also hydrophobic and highly impervious towards chemical and biological degradation. The resistant nature of lignin can be attributed to its complex cross-linked three-dimensional polymeric structure. Moreover, it is located central to the lamella and acts as the binding material for the cell wall and plant cells (Gupta and Tuohy, 2011). Lignin content and composition varies amongst different plant groups rendering possible simple to complex pre-treatment options. Successful lignin fragmentation is key to accessing biologically active compounds of cellulose and hemicellulose for adequate sugar conversion and subsequently production of biofuels. The irregular and complex structure of lignin necessitates a complicated evolutionary pathway to generate a single enzyme that has the capacity of not only recognizing but also disintegrating the different linkages found in lignin (Hatfield and Vermerris, 2001). The linkages found in lignin are inherently 
known to be quite difficult to break down. The structure of lignocellulosic material indicates that lignin covers the hemicelluloses which subsequently cover the cellulose. Accessibility to cellulose is critical as the subsequent hydrolysis of cellulose into sugar can provide necessary sugars for biofuel production. Therefore, lignin fragmentation is a very important step. Pretreatment of lignocellulosic material can remove lignin which can be converted to value-added products such as dyes, pesticides, and industrial plastics (Agbor et al, 2011).

\subsection{Algal Biomass as Feedstock}

Weaknesses of first and second-generation biofuels have urged the development of a newer, more adaptive generation of biofuels. Third-generation biofuels, which are primarily comprised of algae, offer a superior set of characteristics whilst also eliminating the drawbacks associated with its predecessors. Having only being in its infancy stage, algal biomass has been the focal point of numerous scientific studies pertaining to biofuel production (El-Dalatony et al., 2017). Algae can be classified as a diverse set of photosynthetic organisms ranging from simple unicellular cyanobacteria to complex multicellular macroalgae (Gouevia, 2011; State of Technology Review-Algae Bioenergy, 2017; Bulkowska, 2016). Furthermore, algal biomass is rich in carbohydrates, protein, and lipids which ultimately provide an array of biological products including biodiesel, bioethanol, biogas, and biobutanol (El-Dalatony et al., 2017). When compared with terrestrial plants, used for first and second-generation biofuels, algae present lower growth requirements, higher rates of carbon sequestration and improve air quality (Medipally et al., 2015). In terms of their growth requirements, algae cultivation requires nutrients, sunlight, $\mathrm{CO}_{2}$, and water. However, unlike terrestrial plants, algae can be grown using wastewater and nutrients from wastewater sources such as nitrogen and phosphorous (Gouveia, 2011). Another key advantage of using algae as the feedstock for biofuel production, is its 
carbon sequestration ability. Present levels of atmospheric $\mathrm{CO}_{2}$ exceed $400 \mathrm{ppm}$ and are primarily attributed to anthropogenic activities including the combustion of fossil fuels, forestry and land-use changes (Gouevia, 2011; Climate Change Synthesis Report, 2014). Photosynthetic organisms have the capacity to assist in this matter as they can capture and store $\mathrm{CO}_{2}$ as biomass. Algae has been recognized as one of the most effective methods of carbon capture. Furthermore, they have the ability to fix $\mathrm{CO}_{2}$ efficiently from different sources including the atmosphere, industry, and carbonate salts (Wang et al., 2008). When compared with terrestrial plants, the $\mathrm{CO}_{2}$ fixation efficiency of algae is 10-50 times faster; depending on the specific algal strain (Wang et al., 2008). Other advantages of algal biomass for biofuel production is that they provide a higher biomass yield per hectare, grow at higher rates relative to terrestrial plants, and are not restricted to seasonal production (Gouveia, 2011; Medipally et al., 2015).

Due to their recent scientific inquiry in the scientific community and industry, algal biofuels are still considered to be in their development stage. Several demonstration projects have exhibited the vast potential of algae however the scaling-up process is proving to be a difficult task (Zhang et al., 2016; Adeniyi, 2018). Designing a sustainable process that yields high energy returns whilst also being cost-effective are the major roadblocks (Zhang et al., 2016; Adeniyi, 2018). Despite the tremendous opportunity to exploit algae for bioenergy applications, there still remains substantial barriers including technical, economical, and sustainability barriers. Such barriers account for optimizing energy yields, development of algal strains, technological development for efficient extraction methods, and ensuring maximum recycling of nitrogen and phosphorous for growth requirements (Zhang et al., 2016; International Energy Agency, 2017; Adeniyi et al., 2018). 


\subsubsection{Algae Cultivation}

Cultivation of algae is considered a highly significant step in producing a promising and renewable hydrocarbon feedstock for biofuel production. Compared with the growth of other biomass for biofuel production such as a variety of terrestrial plants, algae have the capacity to yield higher energy values, eliminates the reliance upon arable land, and has the capability of using marginal sources of water that are not specifically designated for irrigation or drinking purposes (Ozkan et al, 2012). Despite the limited resurgence of algal biofuel, there are a variety of methods to cultivate algae. The systems to cultivate algal growth can range from open ponds, closed photobioreactors as well as hybrid systems comprised of different elements of various systems (Gouevia, 2011).

Open cultivation systems are reactors that are open to the environment. They have also been recognized as one of the most extensively used and studied forms of algae cultivation (Duran et al, 2018). Within this particular form of cultivation, algal biomass is mechanically mixed with nutrients and water in open-air ponds or circular tanks which are driven by a paddlewheel (Singh et al, 2017). Due to the reliance upon a paddlewheel for the motion of the constituents suspended in water, open cultivation systems for algae are more commonly recognized as raceway ponds. Such cultivation systems have received significant attention and utilization in the laboratory and industrial scale due to their simple construction, cost efficiency, and flexibility (Singh et al, 2017). Figure 2.3 indicates the typical design of a raceway pond. Although raceway ponds have been a popular choice for the cultivation of algae, they do however pose various limitations. One of the prominent disadvantages of raceway ponds is their open interaction with the environmental which can limit the control on growth parameters (Singh et al, 2017). Variances in temperature and $\mathrm{pH}$ can cause issues in the optimization of biomass 
production. Another of raceway ponds is the demand placed upon the natural environment for water and large areas of land to fulfill necessary requirements of production facilities (Singh et al, 2017). Furthermore, open cultivation systems also bring issues of contamination with other algae species and predators and vaporization (Jankowska et al, 2018). Compared with other cultivation methods, open cultivation systems present relatively low concentrations of biomass within the range of 10-25 $\mathrm{g}$ of dry matter of algal biomass per day per square meter (Jankowska et al, 2018).

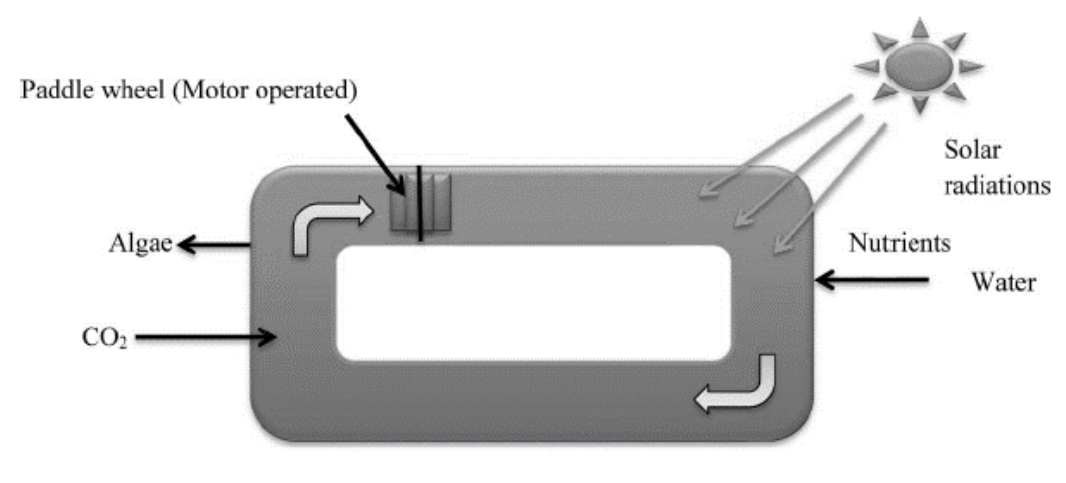

Figure 2.3 Raceway pond schematic (Duran et al., 2018)

Photobioreactors (PBRS) are closed cultivation systems that provide a regulated and controlled environment. PBRS can come in a variety of designs including tubular, flat-tank, bubble column, and serpentine (Jankowska et al, 2018). Figure 2 indicates a tubular structure of a PBR. Furthermore, they exhibit specific characteristics which eliminate the drawbacks of open cultivation systems. As a result of the closed environment, a stronger control of the culture environment can be maintained which can ultimately provide optimal growth requirements. These include the optimal levels of $\mathrm{CO}_{2}$, water, temperature, light exposure, mixing, culture 
density, pH, and gas supply and exchange rate (Menetrez, 2012). PBR systems have higher construction and operational costs associated with the culture process, but their algal cultures are capable of producing a high lipid content biomass (40-55\%) (Menetrez, 2012). Specific conditions are required for optimizing PBRs including the ratio of volume and surface area, the containment to control temperature and pure culture contaminants, and the spatial distribution of fresh air and $\mathrm{CO}_{2}$. Compared with open cultivation systems, $\mathrm{PBR}$ are far more efficient as they can produce a higher biomass content within the range of 2-5 g/L in 2-4 weeks (Duran et al, 2018).

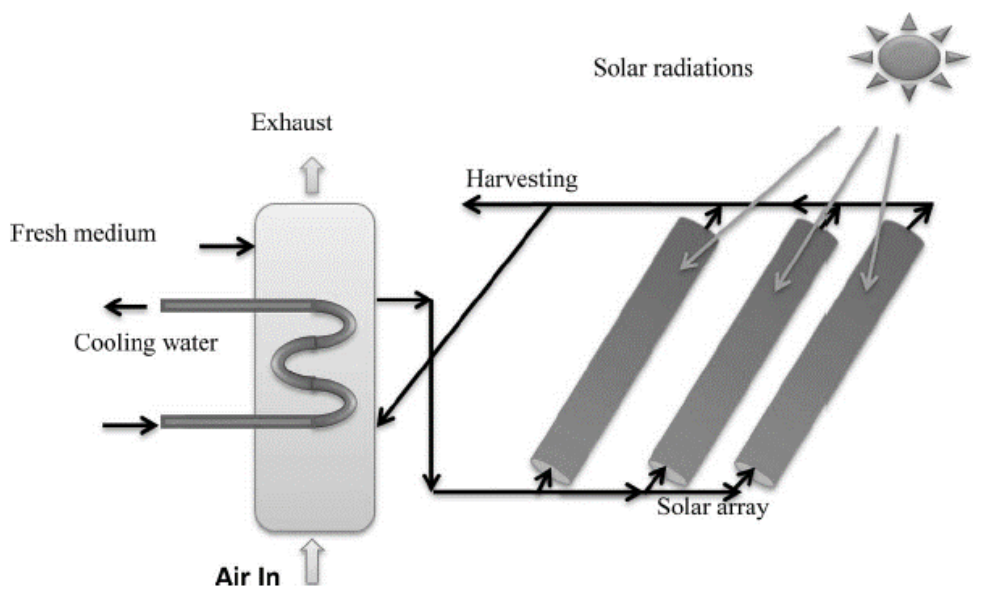

Figure 2.4 Tubular photobioreactor (Duran et al., 2018)

Open cultivation systems are recognized as cost-effective and efficient methods of cultivating algae however they can become easily contaminated with other unwanted species. On the contrast, PBR systems eliminate the interaction with the external environment thereby resulting in a cleaner cultivation environment for algae growth. However, PBR systems result in high construction and maintenance costs. Subsequently, a suitable alternative to achieve sustainable algae cultivation are hybrid systems which bring the optimal elements of both systems. Hybrid systems utilized in biofuel production utilize a large system comprised of both open ponds closed 
bioreactors. Furthermore, to minimize contamination issues proper sanitation methods such as flushing the ponds is carried out. Within hybrid systems, a specified algal species is grown continuously in a PBR and then a portion of that is transferred to an open pond to induce biofuel production (Gouevia, 2011). This process has been successfully utilized at Aquasearch (Hawaii, USA) in which H. pluvialis was cultivated for the subsequent production of astaxanthin (Gouevia, 2011).

\subsubsection{Algae Harvesting}

Upon cultivation, the algae must be separated from the bioreactor effluent. This process is known as algae harvesting. A variety of harvesting processes can be utilized to separate the algae. Harvesting is a critical step in biofuel production and accounts for approximately 20-30\% of the total production cost (Dural et al, 2018). The method chosen, and its rate of success, is dependent upon the respective characteristics of the algal strain used for biofuel production. An ideal harvesting method should utilize few chemicals and low energy. Furthermore, the harvesting method should also aim to cost-effective. Algae harvesting can be dividing into two steps: bulk-harvesting and thickening. During the first step of bulk-harvesting, the algal biomass is separated from the entire culture. Following this, the algal slurry is concentrated. The second step requires more energy when compared to the first step. Different harvesting strategies include sedimentation, centrifugation, flocculation, flotation and filtration.

Sedimentation is a common process for separating algae found in water and wastewater treatment plants. It is a natural process dependent upon the force of gravity. The density and radius of the algae cells along with the sedimentation velocity are influential components for the amount of biomass which settles (Gouevia, 2011). During the sedimentation process, gravitational forces lead liquid and solid particles to become separated from a liquid of different 
density. Sedimentation is an extremely slow process and in environments with high temperatures the biomass has the potential to deteriorate over time. Furthermore, sedimentation can be described by Stokes' Law which explains that the sedimentation velocity is proportional to the square of the radius of the cells and the difference in density between the algal cells and the medium. Various studies have aimed to improve the overall success of this process. Enhancing algal harvesting through gravity-based sedimentation can achieved through lamella separators and sedimentation tanks (Gouevia, 2011).

Similar to sedimentation, centrifugation also relies upon gravitational forces to separate algal cells from the liquid. However, the gravitational forces utilized in centrifugation for separation are significantly greater (Milledge and Heaven, 2012). It has been assessed that almost all forms of algae can be successfully separated through centrifugation. The force applied during centrifugation can be 4000-14,000 times greater than gravitational force (Milledge and Heaven, 2012). Subsequently, this can reduce the separation time significantly. Disc stack centrifugation, which relies upon such strong forces, are most commonly used in industrial practices and are also prevalent in commercial plants for algal products as well as algal biofuel pilot plants (Milledge and Heaven, 2012). Disc stack centrifugation are comprised of shallow cylindrical bowls containing a number of stacked closely spaced metal discs which rotate (Milledge and Heaven, 2012). The mixture, containing the algae, is processed through the centre of the stack of discs. The denser phase is flown out whereas the lighter phase is displaced in the centre (Milledge and Heaven, 2012). The material fed into the centre is then further separated into thin layers and fed through a narrow flow channel of 0.4-3 mm (Milledge and Heaven, 2012). Disc stack centrifuges are typically utilized for separating particle size of 3-30 $\mu \mathrm{m}$. Furthermore, they are capable for separating solid/liquid, liquid/solid, and solid/liquid on a 
continuous basis (Milledge and Heaven, 2012). Due to their high gravitational force required, disc stack centrifuges require significant amounts of energy to operate.

Flocculation is a form of harvesting that aggregates together smaller, finer particles to thus from larger particles more suitable for settling (Gouevia, 2011; Milledge and Heaven, 2012). It has been regarded that flocculation is a form of harvesting that is utilized in conjunction with other harvesting methods (Milledge and Heaven, 2012). Furthermore, it has been recognized that flocculation is a superior method when it comes to handling larger quantities of algae in suspensions (Milledge and Heaven, 2012). Moreover, it has been also been suggested to be the most reliable and cost-effective harvesting technique for algae. Flocculation can occur naturally through auto flocculation and can also be induced through chemicals as a result of chemical coagulation. Naturally occurring flocculation, known as auto flocculation, can take place as a response to environmental stimuli including nitrogen stress, $\mathrm{pH}$ changes, and dissolved oxygen level changes. Furthermore, natural aggregation of particles can also occur as a result precipitation of carbonate salts with algal cells in higher $\mathrm{pH}$ environments. This tends to transpire as a consequence of photosynthetic $\mathrm{CO}_{2}$ consumption with algae (Gouevia, 2011). As such, longer periods of cultivation with the appropriate conditions of sunlight and limited $\mathrm{CO}_{2}$ can help assist in greater capacity of auto flocculation. Naturally occurring flocculation can also be enhanced through the addition of specific chemicals. Chemical flocculation is a process that relies upon the addition of chemicals to the algal culture to promote flocculation (Gouevia, 2011; Milledge and Heaven, 2012). Chemical flocculation can be subdivided into two categories based on the chemical composition: 1) inorganic flocculants and 2) organic flocculants/polyelectrolyte flocculants. 
The flotation harvesting technique for algae is reliant upon the low-density characteristic of algae. It is recognized as a physiochemical gravity separation process in which gas bubbles through the algae containing solution thus enabling the algae to float to the surface. The overall effectiveness of this harvesting technique is dependent upon the size of the particle. Smaller particle sizes work more efficiently in being moved to the top of the solution. Consequently, particles of sizes less then $500 \mu \mathrm{m}$ are best suited for flotation. There are different types of flotation harvesting techniques: 1) dispersed air flotation and 2) dissolved air flotation. Dispersed air flotation is a technique in which an air injection and a high-speed mechanical agitator is used to form bubbles. The gas mixes with the liquid and passes through a disperser which creates bubbles with diameters of 700 to $1500 \mu \mathrm{m}$ (Al Hattab et al, 2015). This method of flotation has been regarded as highly efficient with efficiency greater than 90\% (Al Hattab et al, 2015). Figure 2.5 depicts the typical structure of a dispersed air flotation system.

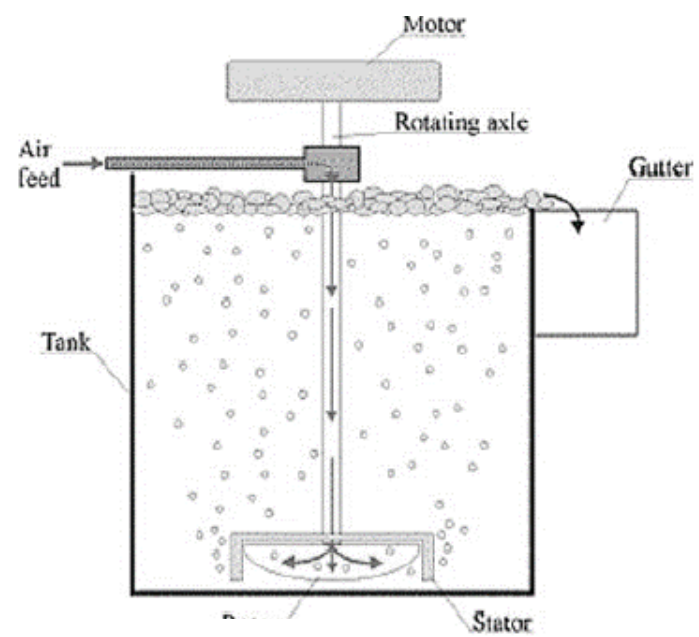

Figure 2.5 Dispersed air flotation technique (Al Hattab et al., 2015) 
Dissolved air flotation is the most commonly used flotation technique, particularly in industrial treatment plants. This particular method is reliant upon water pressure that is presaturated with air. The liquid containing the algal solution is then fed to a flotation tank set at atmospheric pressure and bubbles are subsequently generated through the utilization of a diffuser (Al Hattab et al, 2015). The bubbles then rise through the algal solution carrying with it the algal particles. The accumulated biomass can be collected thereafter. Figure 2.6 indicates the typical structure of a dissolved air flotation system.

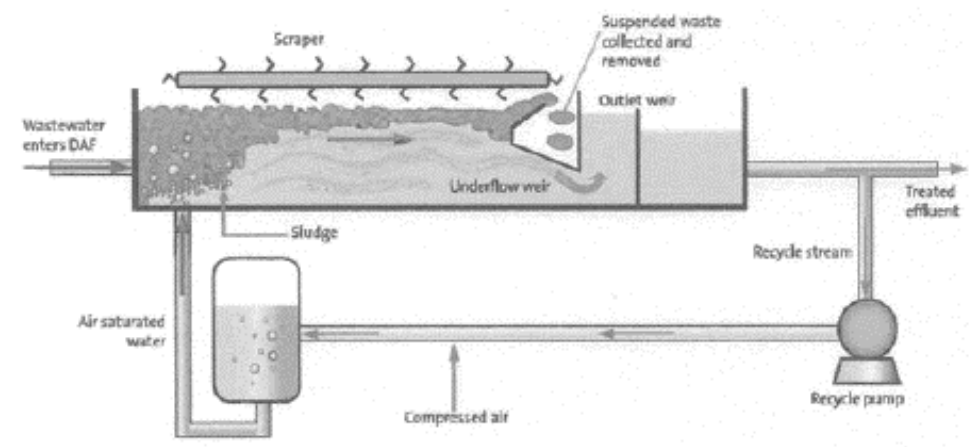

Figure 2.6 Dissolved air flow apparatus (Al Hattab et al., 2015)

Filtration is a type of harvesting technique in which a permeable medium is used to retain the algal biomass whilst allowing the liquid to pass through (Al Hattab et al, 2015; Gouevia, 2011). For the filtration process, a pressure difference is required which can be achieved through a vacuum filter or through gravity. The membrane filters can be categorized by the size of the pores with macro filtration $(>10 \mu \mathrm{m})$, micro-filtration $(0.1-10 \mu \mathrm{m})$, ultrafiltration $(0.02-0.20 \mu \mathrm{m})$ and reverse osmosis $(<0.001 \mu \mathrm{m})(\mathrm{Al}$ Hattab et al, 2015). In general, filtration costs approximately $\$ 10-\$ 20 /$ gallon and has an efficiency ranging from 20-90\% (Al Hattab et al, 2015). Vacuum filtration, pressure filtration, and cross flow filtration are the different forms of filtration used for algae harvesting. The vacuum filtration is a technique that separates the algae 
from the liquid by capturing the solid and pulling the liquid solution through suction.

Furthermore, vacuum filtration is most appropriate for large particles $(>10 \mu \mathrm{m})$. The main benefit of vacuum filtration is that the algal cells are adequately preserved post recovery (Al Hattab et al, 2015). Moreover, this form of harvesting is superior than sedimentation however disadvantages include the period replacement and cleaning of the membrane to avoid clogging. Subsequently, this can increase costs associated with maintenance. Pressure filtration is a process that separates the algae from the liquid in a compact form through pressure. The liquid travels through the membrane filter as a response to a change in pressure (Al Hattab et al, 2015). Advantages of pressure filtration is that the biomass recovered has a low moisture content, and soluble recovery is low ( $\mathrm{Al} \mathrm{Hattab}$ et al, 2015). However, the drawbacks of pressure filtration include the frequent and cumbersome cleaning of the membrane filter (Al Hattab et al, 2015). Lastly, cross flow filtration is a harvesting technique in which the liquid containing algae flows through a membrane and the larger particles are retained in the membrane pores as indicated in Figure 2.7. Subsequently, the smaller particles that flow through are known as permeate. One of the prime benefits of cross flow filtration is that it is a relatively cheap harvesting option. Furthermore, it has been assessed that crossflow filtration is advantageous compared to other harvesting methods including sedimentation, flocculation, and centrifugation as it results in the complete elimination contaminants including debris and the successful separation of algal cells (Al Hattab et al, 2015). Complications of crossflow filtration can arise for large scale industrial applications due to fouling and the recurring replacement of the membrane filter (Al Hattab et al, 2015). 


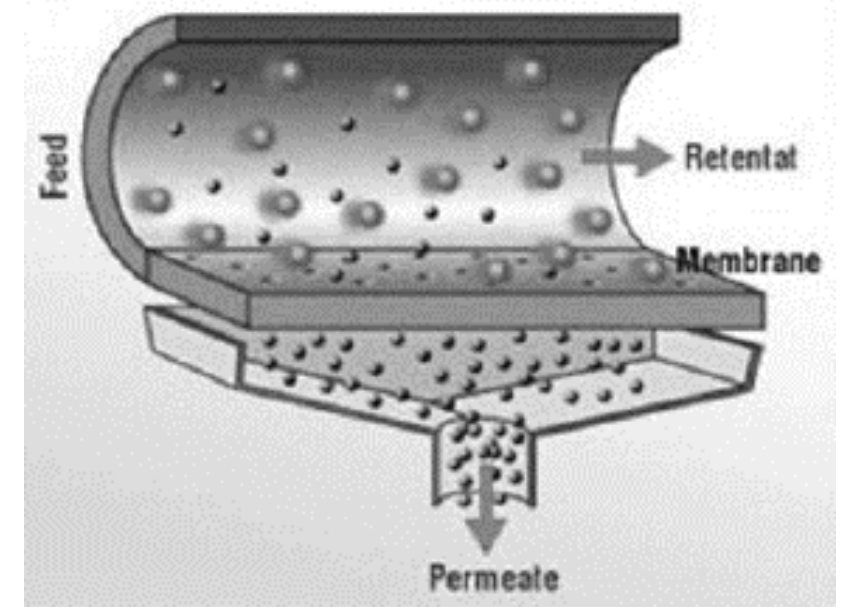

Figure 2.7 Cross flow filtration diagram (Al Hattab et al., 2015)

\subsection{Pre-treatment of Biomass}

The pre-treatment process of lignocellulosic material is a critical step in producing biofuels. Associated techniques of pre-treatment assist in preparing algal biomass for subsequent extraction. Successful pre-treatment should engage in cell wall disruption and increase the bioavailability of biologically active compounds. Microalgae contain three main components including lignin, cellulose, and hemicellulose. The latter two components are comprised of carbohydrate chains that can be converted to simple sugars which can render possible biofuels such as ethanol and butanol. Ultimately, the pre-treatment method incorporated within the biorefinery process must breakdown the lignin structure and aim to expose the cellulose and hemicellulose (Agbor et al, 2011; Günerken et al, 2015). Additionally, pre-treatment should also disrupt the crystalline structure of cellulose, such that acidic or enzymatic hydrolysis action can effortlessly access the cellulose (Kumar et al, 2009). 
Although pre-treatment is considered an essential aspect of the entire biorefinery process as it is directly correlated with higher extraction yields, it is however a costly process. However, advancements of pre-treatment processes to minimize financial costs is a strong element of biofuel research. Subsequently, a variety of pre-treatment methods exist to date that all serve the purpose of simultaneously improving extraction yield and the bioavailability of biologically active compounds within lignocellulosic material. Pre-treatment techniques can be categorized into the following four groups: thermal, mechanical, chemical, and biological. Thermal pretreatments can be further categorized into the following: thermal, hydrothermal, and thermal treatment with steam explosion. Broadly put, all forms of thermal treatments incorporate the solubilization of algal biomass through the application of heat (Passoss et al, 2014). Mechanical treatments can also be further categorized into the following groups: ultrasound and microwave. Such treatments of algal biomass involve the disruption of cell walls through active physical force (Passoss et al, 2014). Chemical treatments can be categorized as acidic or alkali. Chemical treatments utilize chemicals to achieve the solubilization of algal biomass (Passoss et al, 2014). Lastly, biological treatments incorporate the utilization of enzymes to disrupt the cell wall structure of algal biomass.

\subsubsection{Thermal Treatments}

Thermal pre-treatments of algal biomass involve the utilization of heat to disrupt the cell wall structure. The application of heat for pre-treatment of algae as well as other forms of lignocellulosic material has been widely used in literature and in industrial practices. Thermal pre-treatments incorporate high temperatures within the range of $50-270^{\circ} \mathrm{C}$ (Carrère et al., 2010). It has been addressed that choosing the appropriate temperature and subsequent form of thermal pre-treatment is subject to the substrate in use and its associated characteristics. 
However, further studies have indicated optimal temperatures within the range of $150-180{ }^{\circ} \mathrm{C}$ and temperatures above $250^{\circ} \mathrm{C}$ should be strictly avoided as cell wall damage is likely to occur (Passoss et al, 2014).

The application of heat has been considered an effective method to improve the bioavailability of the lignocellulosic material of algal biomass as it increases the solubilization of particulate organic fractions thus allowing the partial hydrolysis of polymeric organic molecules (Rodriguez et al, 2015). Additionally, heat can also play an integral role in also disrupting the lignin and hemicellulose structures of algal biomass. The heat actively interrupts the hydrogen bonds which are situated in the crystalline structure of cellulose and lignocellulose complexes which subsequently causes the algal biomass to swell (Rodriguez et al, 2015). Thermal pretreatments are very commonly used as they also provide a means for sanitization of the respective feedstock to remove any pathogens. Furthermore, thermal pre-treatments are also utilized in conjunction with chemical treatments to enhance the effectiveness.

\subsubsection{Thermal Pre-treatments of Low Temperature}

Distinguished from hydrothermal and steam-based thermal pre-treatments, thermal pretreatments of low temperature-are defined as those that are below $100{ }^{\circ} \mathrm{C}$ and under atmospheric pressure (Passoss et al, 2014; Rodriguez et al, 2015). With the requirement of low temperatures, this form of pre-treatment is advantageous because it requires a lower energy demand relative to high temperature pre-treatments. Upon examination, it has been addressed that the relative performance of thermal pre-treatment is subject to temperature and exposure time (Passoss et al, 2014). Furthermore, when examining different sources of algal biomass, it was suggested that algae grown in wastewater resulted in higher solubilization of lignocellulosic material with temperatures within the range of $75-95^{\circ} \mathrm{C}$ as oppose to lower temperatures of $55^{\circ} \mathrm{C}$. Moreover, 
a longer exposure time of 15 hours as oppose to 10 hours also correlated with higher solubilization of lignocellulosic material.

\subsubsection{Hydrothermal Pre-treatments}

Contrary to the above-mentioned thermal pre-treatments, hydrothermal pre-treatments incorporate temperatures above $100{ }^{\circ} \mathrm{C}$ with a gradual increase in atmospheric pressure (Passoss et al, 2014). Hydrothermal pre-treatments also involve generally shorter exposure times within the range of 15-30 minutes (Passoss et al, 2014). Within hydrothermal pre-treatments the cell walls of algal biomass are broken down and the starch is gelatinized (Velazquez-Lucio et al, 2018). This form of heat-based pre-treatment is widely used in the biorefinery processes of numerous lignocellulosic materials such as algae as well as wheat straw, sugarcane, and softwoods (Velazquez-Lucio et al, 2018). Hydrothermal pre-treatments are subject to reaction catalysts which ultimately speed up the rate of lignocellulosic solubilization. Acid, alkaline, or water reaction catalysts can be applied. Utilizing water as a reaction catalyze has been regarded as a promising technique as it promotes a more sustainable process. Relative to chemical reaction catalysts, water is an environmentally friendly process that results in no toxic waste (VelazquezLucio et al, 2018). Although the utilization of water as a reaction catalyst adheres to a more environmentally friendly process, it does however require significantly higher temperatures and pressures which can only be achieved through the additional use of advanced, costly technologies. Consequently, this increases production costs. It has been indicated that achieving higher temperatures and pressures is strongly correlated with higher levels of gelatinization of starch (Velazquez-Lucio et al, 2018). However, exceeding temperature and pressure thresholds can result in the degradation of starch granules (Velazquez-Lucio et al, 2018). 


\subsubsection{Thermal Pre-treatment with Steam Explosion}

Heat-based pre-treatment with steam explosion is a method that incorporates the increasing of pressure with temperature typically above $160{ }^{\circ} \mathrm{C}$ (Passoss et al, 2014). Following pre-treatment, the pressure can be gradually or rapidly decreased to ambient air pressure which is defined as steam explosion (Passoss et al, 2014). Steam explosion is a commonly used pretreatment technique in the industry and is referred to thermal hydrolysis. During this technique, the respective biomass, such as algae, is placed in a vessel and following this the vessel is subject to high temperatures $\left(>160^{\circ} \mathrm{C}\right)$ and pressures ( $\sim 6$ bars) for an exposure time of within 10-30 minutes. Following this application, the pressure is decreased either gradually or suddenly to achieve ambient air pressure and subsequently rendering possible steam explosion. The so-called steam explosion results in the disintegration of the cell wall and biomass (Passoss et al, 2014; Kepp et al, 2010). Although thermal hydrolysis is a common technique used in the industry, it is still however only under investigation at the laboratory scale for algal biomass. Research has indicated that optimal parameters of thermal hydrolysis incorporate high temperatures in the range of $120-180^{\circ} \mathrm{C}$ and 3-10 bars of pressures (Passoss et al, 2014; Mendez et al, 2014).

\subsubsection{Mechanical Treatments}

The mechanical pre-treatment of algal biomass involves the disruption of cell walls through active physical force. The purpose of mechanical pre-treatment methods is to reduce particle size and crystallinity (Rodriguez et al, 2015). Consequently, this renders possible an increase in the surface to volume ratio that can ultimately shorten and progress the solubilization process (Rodriguez et al, 2015). A variety of mechanical pre-treatment options have been used in literature and in the industry. Such techniques can be categorized into those that rely upon relatively simple techniques and those that rely upon more advanced technologies such as 
ultrasound and microwave pre-treatment methods. All forms of mechanical pre-treatments can be utilized independently or can be combined with other pre-treatment options to enhance the overall pre-treatment process.

\subsubsection{Simple Mechanical Pre-treatments}

Simple mechanical pre-treatment options incorporate chipping, milling, shredding, grinding, and cutting. Upon examination, chipping is the most widely used form of mechanical pre-treatment method for larger lignocellulosic biomass (Rodriguez et al, 2015). On the contrary, grinding and milling are methods that are commonly used for biomass that is compact and rely upon tools such as shredders, knives, scissors, and hammers (Rodriguez et al, 2015). Milling is a common mechanical pre-treatment method used for algal biomass (Passoss et al, 2014). This particular mechanical pre-treatment method can be categorized into for different forms: ball milling, knife milling, disc milling, and hammer milling. With respect to ball milling, the crystalline structure of cellulose is reduced along with the polymerization degree of cellulose and particle size (Rodriguez et al, 2015). Moreover, the bulk density also increases which allows for the pre-treatment of more concentrated biomass and subsequently reduces the reactor volume (Rodriguez et al, 2014). A knife mill can be defined as a rotary piece of equipment comprised of 4-6 knives that are posted on a rotor. The rotor moves at a continuous speed of 500-600 rpm whilst simultaneously cutting the feedstock until it is at an appropriate size to pass through a sizing screen (Rodriguez et al, 2015). A disc mill is a mechanical pre-treatment method in which the biomass is supplied through an orifice coaxially with a rotation axis. A moving disc is pressed against a stationary one which brings in the feedstock to be crushed through pressure, and fictional forces (Rodriguez et al, 2015). Lastly, the hammer mill is comprised of a rotor that 
possesses a set of attached hammers that simultaneously push the material in the appropriate plate to be crushed and shredded (Rodriguez et al, 2015).

\subsubsection{Complex Mechanical Pre-treatments}

Complex mechanical pre-treatment methods involve ultrasound and microwave technologies. Ultrasound mechanical pre-treatment involves the utilization of rapid compressions and decompressions of sonic waves (Passoss et al, 2014). Ultrasound can be defined as acoustic sound energy that resonates in the form of waves which happen to be above a frequency of the human range (Rodriguez et al, 2015). This form of advanced mechanical pre-treatment method calls for the continuous cycles of rapid compressions and decompressions which generate cavitation. Cavitation is known as the formation of specified regions with liquid vapor inside the cell wall which can also be known as micro-bubbles (Rodriguez et al, 2015; Passoss et al, 2014). With the appropriate ultrasound intensity, these micro-bubbles are minimized in size and subsequently implode thereby damaging the cell wall (Rodriguez et al, 2015; Passoss et al, 2014). Through this process, heat, free radicals, high pressure and shockwaves are produced (Rodriguez et al, 2015; Passoss et al, 2014). Parameters of ultrasound pre-treatment methods can be defined according to the substrate being used. Acoustic sound energy has been widely used within the realm of organic chemistry to further facilitate chemical reactions and to increase the bioavailability of biologically active compounds (Velazquez-Lucio et al, 2018). Considering this, a variety of parameters have been tested to determine the effects upon the solubilization of lignocellulosic material. Ultrasound frequencies can be applied at the lower threshold of less than $50 \mathrm{kHz}$ and at the higher threshold of greater than $50 \mathrm{kHz}$ (Passoss et al, 2014). Each threshold results in specified desirable characteristics. For instance, the lower threshold of less than $50 \mathrm{kHz}$ renders mechanical effects whereas the higher threshold of greater than $50 \mathrm{kHz}$ renders the 
formation of free radicals (Passoss et al, 2014). In addition to the frequency, other parameters that are incorporated in ultrasound pre-treatment are the output power and exposure time. The output power is correlated with the biomass concentration.

Microwave assisted mechanical pre-treatment methods are also actively used to disrupt the cell walls of algal biomass. This form of mechanical pre-treatment utilizes short waves of electromagnetic energy with frequencies in the range of $300 \mathrm{MHz}$ to $300 \mathrm{GHz}$ (Passoss et al, 2014; Rodriguez et al, 2015). Such frequencies are a direct result of the rapidly oscillating electric field of a polar or dielectric material (Passoss et al, 2014; Rodriguez et al, 2015;

Günerken et al, 2015). Subsequently, these frequencies are responsible for an increase in kinetic energy that results in a boiling state for water (Rodriguez et al, 2015; Günerken et al, 2015). This process corresponds with the polarization of macro-molecules which subsequently changes the structure of protein and more importantly rapidly generates heat and pressure (Rodriguez et al, 2015; Günerken et al, 2015). This rapid change in heat and pressure ultimately causes swelling of the biomass and forces the biologically active compounds out of their stationary state (Rodriguez et al, 2015; Günerken et al, 2015). Similar to ultrasound, microwave assisted pretreatments can be tailored according to the substrate used in the biorefinery processes. Output power and exposure times, along with frequency, are the main controllable parameters of this mechanical pre-treatment process. Furthermore, with the optimal parameters the native crystallinity of the starch is vanished and subsequently an entirely amorphous material is produced (Velazquez-Lucio et al, 2018; Rozzi and Singh, 2010). Additionally, microwave pretreatments have also been successfully utilized in conjunction with other pre-treatment methods such as enzymatic pre-treatments. Microwave assisted pre-treatments actively promote starch 
digestibility which ultimately enhance the accessibility of enzymes to reach the respective substrate (Velazquez-Lucio et al, 2018; Emami et al, 2012).

\subsubsection{Chemical Treatments}

Chemical pre-treatment methods integrate the utilization of chemical agents to facilitate cell wall disruption and subsequent solubilization of lignocellulosic material. A variety of chemical agents have been used to date to render possible lignin disruption and exposure of cellulose and hemicellulose. Despite this, when compared with thermal and mechanical pretreatment methods, chemical pre-treatments have been used less. Chemical agents can be categorized into acidic or alkali (basic) groups. In both cases, the chemical agents used serves the purposes of solubilizing polymers thus allowing enzyme attack in the subsequent biorefinery process (Passoss et al, 2014). Upon examination, it can be concluded that alkali pre-treatment is best suited for lignin removal whereas acidic pre-treatment works to solubilize cellulose and hemicellulose (Rodriguez et al, 2015). To further enhance the effects of chemical pre-treatments it is generally used in conjunction with thermal pre-treatments.

\subsubsection{Alkali Pre-Treatments}

Within chemical pre-treatment methods, the chemical utilization of alkali methods involves sodium, ammonium, calcium, and potassium hydroxides (Rodriguez et al, 2015). The role of alkaline chemical agents during the pre-treatment process is the saponification and cleavage of lignin-carbohydrate linkages which directly corresponds to increased porosity and surface area (Rodriguez et al, 2015). Subsequently, this decreases the degree of polymerization and crystallinity of the algal biomass (Rodriguez et al, 2015). A beneficial aspect of alkali pretreatment is that it has the capacity to minimize the drop in $\mathrm{pH}$ during acidogenesis (Rodriguez et al, 2015). 


\subsubsection{Acidic Pre-Treatments}

Acidic pre-treatment options are also utilized within chemical pre-treatments of algal biomass. Amongst acidic pre-treatment options the most common chemical agent is sulfuric acid however others have been used including nitric acid, hydrochloric acid, and acetic acid (Rodriguez et al, 2015). Dilute and concentrated acids can be utilized for the pre-treatment of algal biomass. One of the primary benefits of utilizing acidic pre-treatment is that it has the capacity to penetrate through the lignin without need for prior pre-treatment of the biomass (Singh and Trivedi, 2013). Furthermore, this pre-treatment subsequently can breakdown the cellulose and hemicellulose polymers to produce individual sugar molecules (Singh and Trivedi, 2013).

When comparing dilute and concentrated acidic pre-treatments, dilute (1-3\%) options are preferred because they require less acid thus rendering less toxic waste. However, with dilute acidic pre-treatment, thermal assistance is required to enhance the solubilization of the lignocellulosic material. Higher temperatures can facilitate acceptable rates of cellulose conversion to simple sugars (Singh and Trivedi, 2013). On the contrary, concentrated acidic pretreatments (10-30\%) require low temperatures which subsequently produce high hydrolysis yields of cellulose (Singh and Trivedi, 2013). However, upon comparison with dilute treatment options, acidic treatment options hinder the possibility of an environmentally friendly process. With greater amounts of acid required, corrosion problems can arise (Singh and Trivedi, 2013).

\subsubsection{Biological Treatments}

The biological pre-treatment of algal biomass is accomplished through utilization of specific enzymes. Such enzymes have the ultimate purpose of breaking down the compounds of the cell wall into smaller compounds with a lower molecular weight (Passoss et al, 2014). 
Subsequently, this can facilitate anaerobic digestion of the biologically active compounds through bacteria (Passoss et al, 2014). A variety of parameters influence the effectiveness of the enzymatic hydrolysis of algal biomass. These include enzyme dose, exposure time, and temperature (Passoss et al, 2014). Different enzymes can work to breakdown the cell wall structure. Among these are cellulase, xylanase, and $\beta$-glucosidase. Cellulase is an enzyme that targets cellulose and hemicellulose and facilitates its conversion to glucose (Zhao et al, 2016). Xylanase is an enzyme that works to target the polysaccharide structure of xylose which happens to be component of hemicellulose (Zhao et al, 2016). Lastly, the enzyme regarded as $\beta$ glucosidase further facilitates the breakdown of cellulase in the lignocellulosic material of algae (Zhao et al, 2016).

Enzymatic pre-treatments can be combined with different pre-treatment methods including mechanical, thermal and chemical. It has been indicated that with regards to the Rhizoclonium sp. of algae, methane yield increased by $20 \%$ when mechanical pre-treatment was combined with enzymatic pre-treatments (Passoss et al, 2014). Deploying enzymatic pretreatments have also been regarded as environmentally friendly as they require low energy, result in a higher yield of fermentable sugars, operate under relatively light conditions and result in no corrosive problems (Cordova et al, 2018). Upon choosing the appropriate enzyme or mixture of enzymes to target compounds of the cell wall, the following must be considered 1) the compounds that comprise the algae cell wall 2) the optimal ranges for temperature, $\mathrm{pH}$, exposure time and the substrate-enzyme relationship as per enzyme activation and 3) the associated costs of utilizing enzymes (Cordova et al, 2018). 


\subsection{Acetone-Butanol-Ethanol (ABE) Fermentation Process}

The acetone-butanol-ethanol (ABE) fermentation process is a chemical process which relies upon bacterial fermentation to produce acetone, butanol, and ethanol from carbohydrates including glucose and starch. The process depends upon bacteria from the Clostridia family and subsequently produces acetone, butanol, and ethanol in the following ratios: 3 parts acetone, 6 parts butanol, and 1-part ethanol (Gupta and Tuohy, 2011). Clostridia are obligate anaerobe, gram-positive, sporulating firmicutes that can be categorized as pathogenic species to humans and animals as well as non-pathogenic species which are particularly important in the conversion of renewable sources into biofuels and other chemicals (Jones and Woods, 1986; Qureshi et al., 2008). Clostridia species such as $C$. beijernickii $(\mathrm{Cb})$ and $C$. acetobutylicum $(\mathrm{Ca})$ are the most studied biomass-metabolizing bacteria. Furthermore, members of the Clostridia family have the beneficial ability to use both hexose and pentose sugars found in biomass which can be converted to bio-alcoholic fuels (Qureshi et al., 2008). On the contrary, fungi such as yeast, which can also be used in the fermentation process, do not have the ability to hydrolyze the above-mentioned sugars found in biomass (Qureshi et al., 2008).

The ABE fermentation process can be divided into the following two phases: acidogenesis and solventogenesis (Zhang et al., 2016; Wang et al., 2017). During the phase of acidogenesis, acids such as acetic acid and butyric acid are produced whereas during solventogenesis phase the production of solvents (acetone, butanol, and ethanol) occurs (Wang et al., 2016). Figure 2.8 shows the metabolic pathway for which Clostridial cultures breakdown various substrates. Anaerobes such as Clostridia bacteria accumulate sugars through the phosphoenol pyruvate dependent phosphotransferase system (PTS) (Ballongue et al., 1986, Gheshlaghi et al., 2009; Mayank et al., 2012). The hexose sugars are metabolized through the 
Embden Meyerhof pathway (EMP) whereas the pentose sugars are metabolized through the pentose phosphate pathway (PPP) in order to produce pyruvate (Mayank et al., 2012). During the $\mathrm{ABE}$ fermentation process, the most common substrate is starch which is converted to glucose following acid and enzymatic hydrolysis. The pre-treatment process is also responsible for converting other fermentation substrates containing hemicellulose and cellulose to xylose and glucose. Glucose is converted to pyruvate through the EMP pathway. After a series of conversions, xylose is converted to glyceraldehydes-3-phosphate and fructose-6-phosphate which is then ready to enter the EMP pathway (Jones and Woods, 1986; Singh \& Mishra, 1995; Gheshlaghi et al., 2009; Mayank et al., 2012). An exponential growth of cells occurs as a result of the subsequent production of ATP following the fermentation of hexose and pentose sugars. Following the conversion of glucose to pyruvate, the pyruvate is subject to a series of reactions to produce acetyl-CoA. Subsequently, acetyl-CoA follows three separate and simultaneous pathways to produce acetic acid and butyric acid. These two acids have the ability to begin the second phase of solvogenesis as they can permeate the cell membrane. During the solvogenesis phase, acetyl-CoA is converted to acetone with the enzyme phosphate trans-acylase. The enzyme thiolase is then used to convert acetyl-CoA to acetoacetyl-CoA which is subsequently converted to 3-hydroxybutyryl-CoA with the enzyme 3-hydroxybutyryl-CoA dehydrogenase. Following this, the enzyme crotonase converts the previous product to crotonyl-CoA. Crotonyl$\mathrm{CoA}$ is then converted to butyril-CoA with the help of enzyme butyryl-CoA dehydrogenase. Butanol is produced by converting butyril-CoA using the following two enzymes: butyraldehyde dehydrogenase and butanol dehydrogenase (Jones and Woods, 1986). Lastly, the conversion of acetyl-CoA using enzyme ethanol dehydrogenase produces ethanol. 


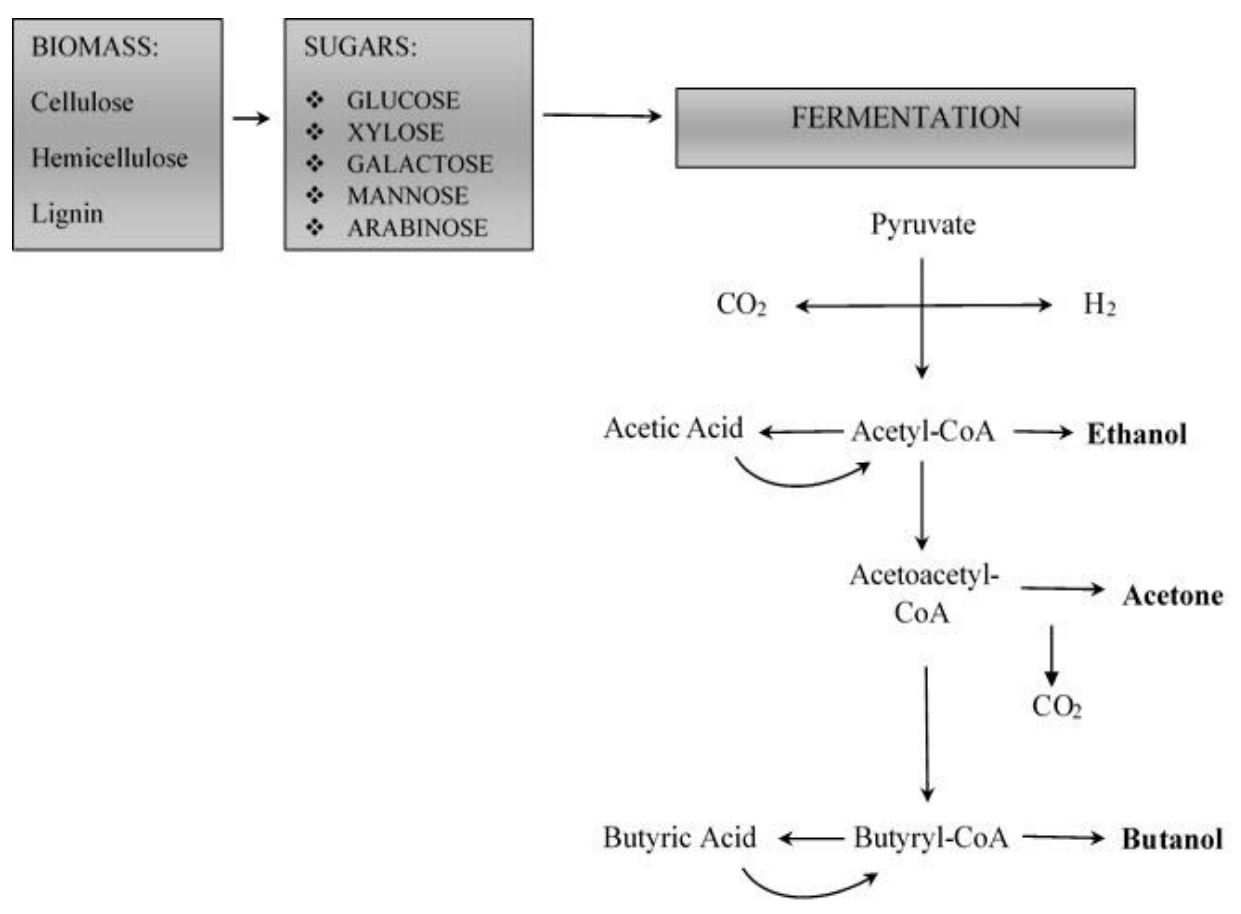

Figure 2.8 ABE Fermentation Pathway (Gupta and Tuohy, 2011)

\subsection{Protoplast Fusion Technology}

Bacteria and fungi are prime components of the fermentation process as they assist in metabolizing biomass to subsequently produce the necessary products that are converted to bioalcoholic fuels such as ethanol and butanol. Furthermore, they are also crucial to the fermentation process as they produce the necessary enzymes that break down substrates. The addition of enzymes increases the overall cost of fermentation however, utilizing the most appropriate enzyme-producing bacteria or fungi has the potential to significantly reduce costs. Therefore, in recent years significant attention has been paid to choose and develop the most appropriate strains of bacteria and fungi for the fermentation process. Both at the industrial scale and the lab scale, single strains of bacteria and fungi have been studied to determine their affect upon the fermentation process and yield. However, little scientific attention has been given to the 
development of hybrid forms of bacteria. Improving algal biofuel production and subsequently increasing its viability as a prime contender in the biofuels market can be a driving force to study the affects of hybrid bacteria. Furthermore, utilizing novel fused bacteria has the potential to improve fermentation yield.

As a result of general scientific interest in manipulating and modifying the genetic make up of an organism for increased versatility and production, there have been a variety of ways to achieve this goal. Somatic hybridization, through protoplast fusion, plays a significant role in ploidy manipulation to improve plant schemes thereby allowing researchers to produce cells of different cultivators, species, or genera (Grosser and Gmitter, 2011). Similar techniques can be applied to bacteria. Protoplast fusion, a genetic engineering technology, renders possible hybrid bacteria with the desirable characteristics of the two bacteria (Kao and Michayluk, 1974; Stanbury et al., 2016). Through the process of protoplast fusion, two different strains of bacteria can be fused together, and potentially produce a higher yield of enzymes required for the simultaneous saccharification and fermentation (SSF) process (Qureshi et al., 2008). Additionally, this further reduces to need for high-priced enzymes.

Protoplast fusion is a three-step process which includes, protoplast formation, fusion, and cell wall regeneration (Kao and Michayluk, 1974). During the fusion process, two cells must be in close proximity to engage in cell wall disintegration and subsequently allow for the joining of the cells. The first stage of the fusion process removes the cell wall of both cells thereby exposing nuclei and forming protoplasts. The next stage requires the fusion of the protoplast which can be carried in five different ways: (1) spontaneous fusion (2) induced fusion (3) mechanical fusion (4) electrofusion and (5) chemofusion. Spontaneous fusion arises when protoplasts fuse together spontaneously which occurs during enzymatic treatment. Adjoining 
cells fuse through their plasmodesmata to subsequently form multinucleate protoplasts. Induced fusion is a form of fusion that utilizes specific chemical agents that reduce the electronegativity of isolated protoplasts thus permitting fusion (Narayanswamy, 1994). Mechanical fusion is a process that promotes intimate physical contact under a microscope with the assistance of a micromanipulator or perfusion micropipette. Electrofusion is a technique that fuses protoplasts together through mild electric stimulations. Chemofusion is a technique that uses several chemicals to induce protoplast fusion. The group of chemicals force the isolated protoplasts to stick to each other and then causes a tight agglutination. Several studies have indicated the successfulness of chemofusion as a technique to fuse together protoplasts. It has been recognized a non-specific and inexpensive method of fusion protoplasts which has the potential to cause large-scale fusion. In previous work conducted in the Nanocomposites and Biomaterials lab at Ryerson University in Canada, have successfully fused together Clostridial strains of $C$. acetobutylicum $(\mathrm{Ca})$ and $\mathrm{C}$. beijernickii $(\mathrm{Cb})$, together with the thermal stable $\mathrm{C}$. thermocellum (Ct) through chemofusion (Thirmal and Dahman, 2011; Mohtasebi, 2013; Begum and Dahman, 2015; Kashif and Dahman, 2015; Ferhan and Dahman, 2016). Fused strains were developed using polyethylene glycol (PEG). Furthermore, ABE concentration, produced through the fused strains, were examined and compared with single strains and co-cultures. It was assessed that the fused strains produced higher concentrations of $\mathrm{ABE}$ when compared to single strains and cocultures. Previous work focused on using novel fusants of $\mathrm{CaCt}$ and $\mathrm{CbCt}$ to produce biobutanol from wheat straw. Furthermore, $C b C t$ was only used to produce biobutanol from algal biomass. ABE production was investigated in SSF using two protoplast fused strains of Clostridia sp. and compared with co-cultures of the bacterial strains. The fusants indicated a higher biobutanol 
yield. In the present study, novel fusants of both $C a C t$ and $C b C t$ were utilized to produce biobutanol using algal biomass.

For more than 100 years, the Clostridia species. $\mathrm{Ca}$ and $\mathrm{Cb}$ have been the prime candidates chosen for cellulose degradation and solvent production during the $\mathrm{ABE}$ fermentation pathway (Cho et al., 2011). The two different Clostridia spp. have successfully facilitated various industrial applications to produce acetone, butanol, and ethanol (Cho et al., 2011; Patakova et al., 2019). Similar to the above-mentioned Clostridia sp., Ctalso has the capacity to secrete a variety of different enzymes that can degrade carbohydrates from different biomass (Lee et al., 1985). Although $C t$ was initially isolated in 1926, it has only recently received significant attention as a viable candidate for use in consolidated bioprocessing (CBP) applications Choosing a Clostridia spp. has been advantageous when compared to other microorganisms used in fermentation processes. Other microorganisms such as Zymononas and modified E. Coli are unable to break down polysaccharides, which are present in lignocellulosic material, into monomeric sugars required for the ABE pathway (Jang et al., 2012). However, utilizing Clostridia sp. for the ABE fermentation pathway has its limitations. Clostridia sp. are subjected to butanol toxicity. High concentrations of butanol can hinder the bacteria to maintain an internal $\mathrm{pH}$, reduce the intracellular level of ATP, and also inhibit glucose uptake (Jang et al., 2012). Studies have indicated $C a$ and $C b$ have a butanol tolerance of up to $13 \mathrm{~g} / \mathrm{L}$ (Pfromm et al., 2010).Ct, on the other hand, has been noted to have a butanol tolerance of $5 \mathrm{~g} / \mathrm{L}$ however various mutated forms of $C t$ can vary from this value (Pfromm et al., 2010 and Tian et al., 2019). Improving butanol tolerance while also maintaining efficient cellulose degradat2ion rates is a great challenge for several industrial practices relying upon the $\mathrm{ABE}$ fermentation process. Consequently, genetic manipulation of different Clostridia sp. can assist in deriving a suitable 
candidate for the process. As this study can indicate, through protoplast fusion, the butanol tolerance, cellulose degradation rate and thermophilic capacity was improved.

\subsection{Biodiesel Production Process}

Biodiesel production requires the transesterification of the oil content found in biomass. It is generally a two-step process that involves the extraction of oil followed by a conversion process that produces biodiesel. A purification stage can be followed to separate and purify the desired extracted materials (Amin, 2009). Due to its vast presence in the biofuels market, biodiesel production has experienced an array of transformations and has consequently been the centre of research and development. Such research and development initiatives have and continue to explore how each stage of biofuel production can be improved. Consequently, there a variety of different methods and techniques to extract oil from biomass with recent studies fixated upon algae. The most common methods of oil extraction can be categorized into two groups: mechanical and chemical. Mechanical methods include following: the use of expellers, ultra-sound assisted extraction, and microwave-assisted extraction. Chemical methods can be classified into the following: solvent-based method and supercritical fluid extraction. Oil extraction from algae can be carried out through single methods or a combination of these methods as well. Extracting oil from algal biomass is a critical step during the biodiesel production process as this step greatly influences the overall yield, cost, and feasibility of the entire process (Derakshan et al., 2014). Although there have been numerous laboratory-scale protocols to remove oil from algal biomass there is still no standard procedure that is followed at the industrial scale. Furthermore, each of the following methods have their advantages and disadvantages which will be described below. 


\subsubsection{Algal Oil Extraction Methods}

\subsubsection{Mechanical Extraction}

1) Expeller:

The expeller method is the simplest and oldest way to extract oil from biomass. This mechanical method incorporates a physical pressing of the dry algal biomass using an oil press (Demirbas, 2009; Kumar et al., 2015). The goal of this application is to apply high-mechanical pressure for crushing the algal biomass which subsequently breaks the cell walls and thus squeezing the oil out of the biomass (Kumar et al., 2015). Based on the algal strain and lipid content, specific pressure ranges have the potential to improve extraction efficiency however too much pressure can result in a decline in lipid recovery as well as heat generation and choking issues (Demirbas, 2009; Ramesh, 2013). Due to the varying algal characteristics and oil content, which are based on differences in morphological features, different oil presses can be configured based on screw, expeller, and piston sizes (Kumar et al., 2015). In many cases, this method is used in conjunction with other techniques to enhance recovery. An integral component of the expeller method is to ensure low-moisture content which is an energy intensive process and can account for up 30\% of the total production costs (Kumar et al., 2015). Although the expeller process is one of the oldest and simplest methods of oil recovery, it is expensive and time consuming. Furthermore, with respect to algal biomass, oil recovery can require significant amounts of energy when compared with oil seeds. In contrast, algae cells have more rigid cell walls which consequently require more energy for disruption. Additionally, the expeller process adds to the cost of extraction due to the necessary step of removing pigments before full oil conversion process. Other disadvantages of the expeller method are high-maintenance costs, 
requirement of skilled labor, and less efficiency when compared with other oil extraction methods (Demirbas, 2009; Ramesh, 2013; Kumar et al., 2015).

\section{2) Ultrasound Extraction:}

Recent research has indicated the potential suitability of using ultrasound waves to extract oil from different biomass including algae. This extraction method uses ultra-sound waves continuously or in pulses to disrupt and cause damage to algae cells. Subsequent waves impede the cells and result in the production of microbubbles; a process known as cavitation. Simultaneously, the ultra-sound waves increase the pressure over time and thus with pressure build-up the microbubble burst, causing the cell walls to subsequently break (Kumar et al., 2015). The ultrasound waves can generate transient and stable cavitation as there is consistent rapid compression and decompression cycles. Unsteady oscillations can result in the cells eventually imploding (Kumar et al., 2015). Consequently, the cavitation result in heat shock waves which can disrupt the cells and cause cracks in the cell walls. Studies have indicated that this method is simple, economical, and environmentally friendly. Another advantage of ultrasound assisted extraction is that it generates relatively low temperatures when compared with microwave reactors and autoclaves thus leading to less thermal denaturation of biomolecules. One of the drawbacks of ultra-sound assisted extraction is prolonged extraction time which can lead to the production of free radicals that can be detrimental to the quality of the oil (Kumar et al., 2015).

\section{3) Microwave Extraction:}

The microwave extraction method was initially developed in the early 1980s. During this application, a dielectric or polar material is placed in a rapidly oscillating electric field which 
will generate heat due to frictional forces (Kumar et al., 2015; Amarni and Kadi, 2010). The heating results in the production of water vapor which disrupts the cell walls. Consequently, the permeability of the cells increases which also increased heat and pressure thus forcing biological compounds out from the cell matric (Kumar et al., 2015). Like the ultra-sound extraction method, the microwave extraction method requires a high initial investment and the costs associated with maintenance are also high. Furthermore, this method is also energy intensive. Although this application has been recognized as easy to scale up it still requires standardization at the commercial level (Kumar et al., 2015).

\subsubsection{Chemical Extraction}

\section{1) Solvent Extraction:}

Lipid extraction through this method is carried out through the application of solvents. The use of solvents assists in the degradations of lipids and their various interactions (Pragya et al., 2013). Non-polar solvents can disrupt hydrophobic interactions between non-polar/neutral lipids. Polar organic solvents act similarly to alcohols in which they have the ability to disrupt hydrogen bonding between polar lipids (Pragya et al., 2013). The solvent extraction method is commonly used with the Soxhlet extractor or the Bligh and Dyer method. The Soxhlet extractor method most commonly uses hexane whereas the Bligh and Dyer method rely upon a mixture of chloroform and methanol as the solvents. However, recent scientific assessment of these two methods has raised significant environmental concerns and risks with respect to the utilization of toxic chemicals (Kumar et al., 2017).

The Bligh and Dyer method was initially introduced in 1959 as a procedure to study lipid decomposition in frozen fish (Sundermann and Schwudke, 2011; Breil et al., 2017). Since then, it is a standard procedure to isolate and remove lipid fractions from biological matrices utilizing 
chloroform, methanol, and water in the following ratio 2:2:1.8 (v/v/v) (Sundermann and Schwudke, 2011; Kumar et al., 2015; Breil et al., 2017). The procedure initially homogenizes a biological sample in a monophasic chloroform/methanol mixture with a ratio of 1:2. Following this, the suspension is vortexed and additional chloroform and water are added. The resulting suspension contains non-extractable residues in a chloroform/methanol/water mixture in ratio of $2: 2: 1.8(\mathrm{v} / \mathrm{v} / \mathrm{v})$. Following filtration and a short incubation period, the filtrate is entirely phase separated. The upper phase contains polar molecules (salts and amino acids) and the bottom phase contains the non-polar lipid phase (Sundermann and Schwudke, 2011; Kumar et al., 2015; Breil et al., 2017).

The Soxhlet extractor (Figure 2.9) is a simple laboratory equipment invented by Franz von Soxhlet in 1879. It is a commonly used method to remove oil from solid biomass. It has been described as a very useful tool for preparative purposes that incorporates a procedure in which the analyte is concentrated from a matrix as a whole or separated from particular interfering substances (Castro and Ayuso, 2000). Removing oil from a solid biomass is commonly referred to as a solid-liquid extraction and it is one of the oldest methods of extraction. Furthermore, Soxhlet extraction is one of the most relevant and commonly used techniques for oil extraction (Castro and Ayuso, 2000). The apparatus contains three main parts (1) a continuously heated round distillation flask (2) a Soxhlet extractor to hold the biomass and (3) a condenser for continuous cooling (Mubarak et al., 2015). During the extraction process, the solvent is initially heated to reflux, and the solvent vapors travel up to the distillation arm then to the condenser. The condenser ensures that the vapors cool down and subsequently experience a phase change from vapor to liquid. The liquid solvent floods the chamber holding the thimble containing the solid biomass. During this stage, the warm solvent begins to react with the solid biomass to 
extract oils. Once the chamber is full, it is emptied through the siphon and the solvent is then returned to the distillation flask. This cycle can be completed several times for hours or days. During each cycle, a portion of the non-volatile compound (oil) is transferred into the distillation flask along with the solvent. Hexane is commonly used as the prime solvent for Soxhlet extraction (Pragya et al., 2013). Various studies have executed the Soxhlet extraction using hexane for lipid recovery (Castro and Ayuso, 2000; Mubarak et al., 2015; Naghdi et al., 2016). However, extraction times can be very time consuming and the scaling-up process has been seen a difficult task (Mubarak et al., 2015)

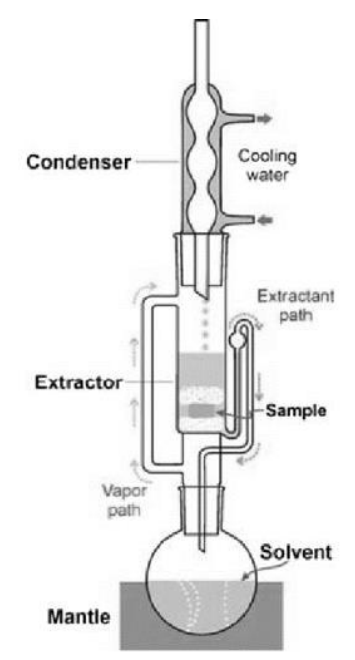

Figure 2.9 Soxhlet Apparatus (Dabbs et al., 2006)

Although lipid recovery from Bligh and Dyer and the Soxhlet extraction can be relatively high, the use of toxic solvents such as hexane and chloroform cause adverse health and environmental hazards (Mubarak et al., 2015). Consequently, a variety of alternative solvents have been studied to determine their feasibility (Tanzi et al., 2012 and Mubarak et al., 2015). Tanzi et al. (2012) reported a two-step process of using terpenes for oil extraction in microalgae. Terpenes are defined as biologically active compounds that are extracted from plants and citrus peels including d-limonene, p-cymene, and gum turpentine. D-limonene is the most commonly 
used terpene investigated in literature. When studied with algal species, Chlorella vulgaris, the oil yield was comparable with hexane. This incorporated Soxhlet extraction using terpenes followed by a Clevenger distillation method for solvent elimination. This study indicated similar values of oil extraction from hexane and d-limonene. Oil yield from hexane, p-cymene, and dlimonene were $0.88 \%, 1.52 \%$, and $1.29 \%$; respectively.

The first attempt to use d-limonene as a solvent was used to extract oil from rice bran (Mamidipally et al., 2004). The process, dependent upon d-limonene, was able to deliver a slightly higher yield than hexane. Studies with olives and marine microalgae were able to indicate comparable results with hexane. The higher polarity of d-limonene when compared with hexane allows it to extract a higher amount of lipids. Therefore, this indicates the potential of terpenes in replacing hexane. Other studies have examined the potential of different terpenes such as a-pinene, and p-cymene. D-limonene can also be up to $90 \%$ recycled whereas hexane can only be recycled up to $50 \%$ thereby indicating the economic value of d-limonene (Mamidipally et al., 2004 and Tanzi et al., 2012).

\section{2) Supercritical Fluid Extraction:}

The supercritical fluid extraction (SFE) method is a process that separates one substance (extractant) from another (matrix) with the assistance of supercritical fluids which act as the primary solvent (Kumar et al., 2017). This technology has been quite relevant in food and pharmaceutical industries over the past several years. Recent assessment of this technology has sparked great interest in various other applications including microalgae lipid extraction (Kumar et al., 2017). This technology uses supercritical fluids, which are above their critical point to extract a solvent from a solid biomass. In thermodynamics, the critical point is known as an end point at which two phases of a substance begin to be indistinguishable from each other. At this 
point, the substance is above its critical temperature and pressure thus enabling the production of a supercritical fluid. A supercritical fluid is known as a substance that is not a distinct liquid or gas. It simultaneously exhibits liquid-like properties as well as gas-like properties. During microalgae oil extraction, studies have primarily looked at $\mathrm{CO}_{2}$ as the supercritical fluid. $\mathrm{CO}_{2}$ has been the prime choice of substance for this technology as it is odorless, colourless, very pure, safe, cost-effective, non-toxic, non-flammable, and can be recyclable at low pressures and temperatures (Capuzzo et al., 2013). During the process, microalgae is placed in a vessel or stainless steal tank and $\mathrm{CO}_{2}$ is injected inside. Initially the $\mathrm{CO}_{2}$ is under high pressure thus resulting in a liquid state and subsequently begins to remove the oil from the algae. Upon decreasing the pressure, $\mathrm{CO}_{2}$ transitions back to its original gaseous phase and the oil extract settles out of the algal biomass (Capuzzo et al., 2013; Pragya et al., 2013; Kumar et al., 2017).

This technology has been recognized as a sustainable, green alternative method for oil extraction. It has the potential to be a viable contender in replacing volatile, harmful, and environmentally risky chemicals used in other applications (Kumar et al., 2017). However, one of the primary drawbacks of this technology is its significantly high costs. Furthermore, this application is very energy intensive. Additionally, due to the lack of industrial protocol, specific to microalgae lipid recovery, the technology still poses a degree of safety risks that have yet to be minimized or eliminated (Pragya et al., 2013; Kumar et al., 2015; Kumar et al., 2017).

\subsubsection{Transesterification}

Transesterification is a biochemical process that converts the lipid fraction of a biomass into biodiesel. Due to the high lipid content of algae, biodiesel has been a highly valuable product produced through algae. The transesterification process involves the exchange of an alkoxy group of an ester with another alcohol (Amin, 2009). Furthermore, the process 
incorporates the utilization of acids or bases, which act as catalysts that essentially facilitate the reaction between a fat or oil and an alcohol to form esters and glycerol (Amin, 2009). Biodiesel is a biofuel that is comprised of methyl esters that are formed through process indicated in Figure 2.10 Methanol is commonly used as the alcohol to facilitate the reaction coupled with a catalyst in the form of $\mathrm{KOH}$ or $\mathrm{NaOH}$ (Demirbas, 2010). During transesterification, there are four main process that occur: 1) the triglycerides, alcohol (methanol) and catalyst $(\mathrm{KOH}$ or $\mathrm{NaOH})$ are placed in a controlled chamber to carry out transesterification 2) the initial product is placed in a separator to remove the glycerin byproduct 3) the excess methanol is recovered through evaporation and if possible reused and 4) final biodiesel product is rinsed with water and neutralized (Demirbas, 2010).

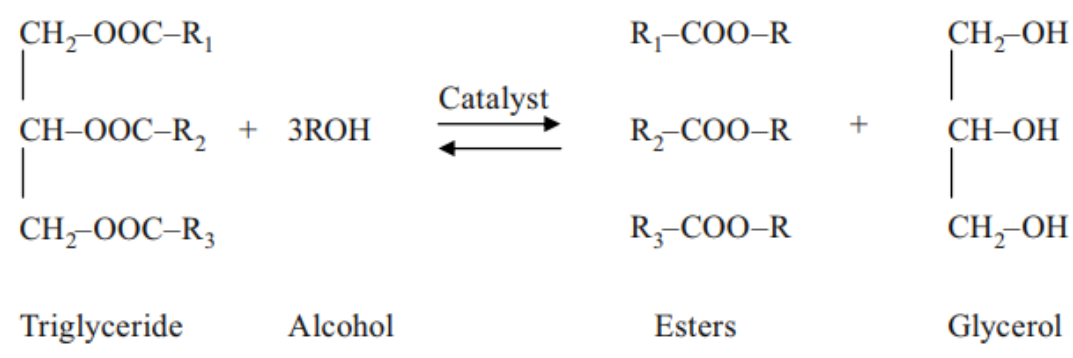

Figure 2.10 Transesterification reaction scheme of triglycerides with alcohol

A variety of successful applications of transesterification at the commercial scale have been able to produce economically viable biodiesel. Due the vast potential of biodiesel, this fuel has been experienced significant growth and development over the years. The United States and the European Union are the world's leader in biodiesel production with production rates at 6.65 billion litres in 2014 and 11.77 billion litres in 2013; respectively (Knothe and Razon, 2017). 
The energy density of biodiesel also comparable to that of petroleum-based diesel. Furthermore, biodiesel is considered a viable contender against conventional diesel as it is renewable, readily available, has a higher combustion efficiency, as well as a low sulfur content and aromatic content (Demirbas, 2010). Additionally, in terms of air pollution, biodiesel does not contribute to atmospheric $\mathrm{CO}_{2}$ emissions. Furthermore, besides NOx emissions that happen to be lower than petroleum diesel, results in a significant reduction in particulates and carbon monoxide emission as well (Demirbas, 2010). Although the utilization of biodiesel is efficient and sustainable, the process of producing it has received criticism recently. Oil extraction and transesterification process require harsh chemical such as hexane and methanol, which are potentially problematic as they pose environmental and safety risks as well as bring up issues of sustainability. 


\section{CHAPTER 3: \\ MATERIALS AND METHODS}

\subsection{Chemicals and Materials}

Three different bacteria strains were utilized in the present study; Clostridium beijernickii (ATCC BA101) (Cb), Clostridium acetobutylicum (ATCC 4259) (Ca), and Clostridium thermocellum (ATCC 27405) (Ct). $C a$ and $C b$ were purchased from the American Type Culture Collection (ATCC). Ct was generously provided by Dr. Wolfaardt's lab at Ryerson University. All the chemicals utilized in the present study were purchased from Sigma-Aldrich Canada; Appendix A. The algae utilized in the present study was provided by Pond Technologies located in Markham, Ontario. The alga was classified as the freshwater strain of Stichococcus. The freshwater alga was grown in photobioreactors with an optimal temperature of $30^{\circ} \mathrm{C}$ with varying amounts of nutrients (nitrogen and phosphorus) depending upon the specific batch. Furthermore, the photobioreactors utilized a red LED light source for algal growth.

\subsection{Experimental Procedures}

\subsubsection{Clostridia Strain Development}

Prior to strain development and subsequent fusion, bacterial strains required appropriate culture conditions as a means to multiply the microbial organisms. As per the ATCC, Reinforced Clostridial Medium (RCM) was the predetermined cultural media to allow for the reproduction of $\mathrm{Ca}$ and $\mathrm{Cb}$. RCM was prepared by adding $13.0 \mathrm{~g}$ yeast, $10.0 \mathrm{~g}$ peptone, $5.0 \mathrm{~g}$ glucose, $1.0 \mathrm{~g}$ starch, $5.0 \mathrm{~g} \mathrm{NaCl}$ (sodium chloride), $3.0 \mathrm{~g} \mathrm{CH}_{3} \mathrm{COONa}$ (sodium acetate), and $0.5 \mathrm{~g}$ $\mathrm{C}_{3} \mathrm{H}_{8} \mathrm{CINO}_{2} \mathrm{~S}$ (cysteine hydrochloride) in 1 litre of distilled water. The solution was then boiled, and $0.5 \mathrm{~g}$ of agar was added. Following this, the solution was then subjected to $\mathrm{N}_{2}$ gas bubbling. Finally, the solution was autoclaved at $120^{\circ} \mathrm{C}$ for 20 minutes. Bacteria pellets of $\mathrm{Ca}$ and $\mathrm{Cb}$, 
provided by ATCC, were revived in the RCM solution. $5 \mathrm{~mL}$ of the solution was anaerobically transferred to test tubes. $0.5 \mathrm{~mL}$ of the RCM solution was added to the pellet and then the bacteria pellet was transferred to the test tube with $5 \mathrm{~mL}$ RCM. The test tube was mixed thoroughly and incubated at $37^{\circ} \mathrm{C}$ overnight. This process was repeated again for the other bacterial strain. $C t$ was received in a culture media. All procedures were carried out in an anaerobic glove box (Terra Universal, Canada) at an average temperature of $25^{\circ} \mathrm{C}$ with a constant flow of $\mathrm{N}_{2}$ gas. Once sufficient growth and turbidity of growth media for $C a$ and $C b$ was achieved, the strains were then plated on agar petri dishes along with $C t$. The three bacterial strains were incubated in the petri dishes at $37^{\circ} \mathrm{C} . \mathrm{Ca}$ and $\mathrm{Cb}$ achieved adequate growth of colonies within 3-4 days whereas the $C t$ colonies began to develop within 6-7 days. Colonies were transferred anaerobically into Eppendorf tubes with $30 \%$ v/v sterile glycerol and RCM. The Eppendorf tubes were stored in a $-82^{\circ} \mathrm{C}$ freezer (Thermo Fisher Scientific, USA) until protoplast fusion.

\section{Protoplast Fusion}

The three parent strains of bacteria $(\mathrm{Ca}, \mathrm{Cb}$, and $C t)$ were subject to protoplast fusion to form $\mathrm{CaCt}$, and $\mathrm{CbCt}$. Previous work conducted in the Nanocomposites and Biomaterials Lab at Ryerson University developed novel fusants using the protoplast fusion technique. The fusion process has been described by Thermal and Dahman (2011), Begum and Dahman, (2015), Syed and Dahman (2015), and Ferhan and Dahman (2016). The protoplast fusion process is comprised of three main steps which included protoplast formation, fusion, and cell wall regeneration (Kao and Michayluk, 1974). For the first part of the fusion process, overnight cultures of the three parent strains were diluted with 1:4 fresh, sterile Clostridia Basal Medium (CBM) containing $0.4 \%$ glycerol. CBM was prepared by adding $2.0 \mathrm{~g}$ glucose, $0.04 \mathrm{~g} \mathrm{MgSO}_{4}$ (magnesium sulfate), 


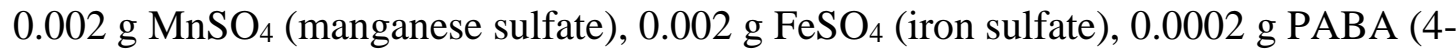
aminobenzoic acid), $0.004 \mathrm{~g}$ biotin, $0.0002 \mathrm{~g}$ thiamin $\mathrm{HCl}$, and $0.8 \mathrm{~g}$ casein hydrolysate in 200 $\mathrm{mL}$ distilled water. The solution was autoclaved at $120^{\circ} \mathrm{C}$ for 20 minutes. After approximately 45 minutes, the cells began to experience $100 \%$ motility and were then centrifuged at $10,600 \mathrm{~g}$ for 10 minutes. Following this, the osmotic strength of each culture was strengthened by adding $5 \mathrm{~mL}$ protoplast medium (PPM). PPM was prepared by adding CBM, $0.3 \mathrm{M}$ sucrose, $50 \mathrm{mM}$ $\mathrm{CaCl}_{2}$ (calcium chloride), and $50 \mathrm{mM} \mathrm{MgCl}$ (magnesium chloride) in $100 \mathrm{~mL}$ distilled water. The $\mathrm{pH}$ of the solution was then adjusted to $7.5 \mathrm{using} 2.5 \mathrm{mg} / \mathrm{mL}$ of lysozyme (Reily and Rogers, 1987). The bacteria cultures were incubated for approximately 60 minutes at $35^{\circ} \mathrm{C}$ for $\mathrm{Ca}$ and $\mathrm{Cb}$ and $45^{\circ} \mathrm{C}$ for $C t$. After approximately 60 minutes, the protoplasts were extracted through centrifugation at 3,300 rpm for 5 minutes. Following this, the protoplasts were put in fresh PPM and the cell pellets were stored in the fridge until the next step.

The second step, involving the fusion process, required the mixing of $C a, C b$, and $C t$ as followings: $C a+C t$ and $C b+C t$. The cell pellets of $C a$ and $C t$ were mixed together and then centrifuged at $1,500 \mathrm{~g}$ for 10 minutes. Subsequently, the cell pellets of $C b$ and $C t$ were mixed together and centrifuged at 1,500 for 10 minutes. The pelleted protoplasts were then placed in 1 $\mathrm{mL}$ of PEG(40\% w/v) and PPM for approximately 2-3 minutes.

In the next and final step of the protoplast fusion process, the cell wall is regenerated. Dilutions of the protoplasts were plated into a Regeneration Medium (RM). The RM was prepared by making 5 different stock solutions (A-E) and a basal mixture. Stock A was prepared by adding $0.1 \mathrm{~g}$ biotin, $0.1 \mathrm{~g}$ PABA (4-aminobenzoic acid), $0.001 \mathrm{~g}$ Thiamin $\mathrm{HCl}, 0.1 \mathrm{~g} \mathrm{FeSO}_{4}$ (iron sulfate), $0.1 \mathrm{~g} \mathrm{MnSO}_{4}$ (manganese sulfate), and $0.2 \mathrm{~g} \mathrm{MgSO}_{4}$ (magnesium sulfate) in 100 $\mathrm{mL}$ distilled water. Stock B was prepared by adding $25 \mathrm{~g}$ glucose in $100 \mathrm{~mL}$ distilled water. 
Stock $\mathrm{C}$ was prepared by adding $2.5 \mathrm{M} \mathrm{MgCl}_{2}$ (magnesium chloride). Stock $\mathrm{D}$ was prepared by adding $2.5 \mathrm{M} \mathrm{CaCl}_{2}$ (calcium chloride). Stock $\mathrm{E}$ was prepared by adding $7.0 \mathrm{~g} \mathrm{~K}_{2} \mathrm{HPO}_{4}$ (dipotassium phosphate) and $3.0 \mathrm{~g} \mathrm{KH}_{2} \mathrm{PO}_{4}$ (monopotassium phosphate) in $100 \mathrm{~mL}$ distilled water. The basal mixture was made by adding $50.0 \mathrm{~g}$ gelatin, $15.0 \mathrm{~g}$ agar, $8.0 \mathrm{~g}$ yeast, $2.5 \mathrm{~g}$ casamino acids, and $1 \mathrm{~g}$ asparagine in $900 \mathrm{~mL}$ distilled water. The basal mixture was boiled and autoclaved at $120^{\circ} \mathrm{C}$ for 20 minutes. The RM was prepared as follows: $10 \mathrm{~mL}$ Stock A, $40 \mathrm{~mL}$ Stock B, $5 \mathrm{~mL}$ Stock C, $5 \mathrm{~mL}$ Stock D, $10 \mathrm{~mL}$ Stock E and the basal mixture. The fused protoplasts were plated on petri dishes with RM. The petri dishes were incubated at $45^{\circ} \mathrm{C}$ for approximately 1-2 days. The regenerated colonies were then extracted from the petri dishes and placed in CBM with $30 \%(\mathrm{v} / \mathrm{v})$ glycerol. Following this, the fused strains were stored in Eppendorf tubes and kept in a $-82^{\circ} \mathrm{C}$ freezer.

\section{UV Mutagenesis}

A mutagenesis study involving UV light was conducted on the fused strains of $\mathrm{CaCt}$ and $\mathrm{CbCt}$. The fusants were also analyzed for their growth rate in the presence of $15 \mathrm{~g} / \mathrm{L}$ of biobutanol to determine tolerance to biobutanol toxicity. Following the formation of protoplasts, the fusants were extracted, placed in CBM and subsequently plated on petri dishes and were grown for 1-2 days. The petri dishes were then placed inside the anaerobic glove box to carry out the UV mutagenesis study. The surface of the petri dishes containing the fusant were placed at distance of approximately $6 \mathrm{~cm}$ from a UV-light source (245 nm) (Bowring and Morris, 1986). Prior to the use of the UV-light, a control sample of 100 microlitres was taken and plated on an agar petri dish. Then the fusant was subjected to UV-light exposure for time intervals of 5 minutes for 25 minutes. To determine the ability to grow and subsequent tolerance of the fusants in the presence of biobutanol, another 100 microlitre sample was taken after each 5-minute 
interval and plated on agar plates containing $15 \mathrm{~g} / \mathrm{L}$ of butanol. The petri dishes were incubated overnight, and the cell count was determined the following day. Raw data for the cell concentrations following UV mutagenesis can be found in Appendix B.

\subsubsection{Pre-treatment of Algae}

Algae were subject to different pre-treatments including chemical, thermal, and enzymatic. All samples were subject to physical treatment to increase surface area. 2 grams of algae was ground into a fine powder using a mortar and pestle. Chemical treatments consisted of $1 \% \mathrm{H}_{2} \mathrm{SO}_{4}$ and $2 \% \mathrm{H}_{2} \mathrm{SO}_{4}$. Enzymatic treatments included the use of cellulase and $\beta$-glucosidase. Combination treatments of acid and enzymes were also applied. Each treatment was replicated with a thermal treatment. A control treatment was also applied as a point of comparison. Table 3.1 indicates the different treatments and their details.

Table 3.1 Hydrolysis treatments of algal biomass

\begin{tabular}{|c|c|}
\hline Treatment & Details \\
\hline 1. Control & Algae with $\mathrm{H}_{2} \mathrm{O}$ \\
\hline 2. Control + Thermal & Algae with $\mathrm{H}_{2} \mathrm{O}$ subject to $121^{\circ} \mathrm{C}$ for 20 minutes \\
\hline 3. Chemical & Algae with $1 \% \mathrm{H}_{2} \mathrm{SO}_{4}$ \\
\hline 4. Chemical + Thermal & Algae with $1 \% \mathrm{H}_{2} \mathrm{SO}_{4}$ subject to $121^{\circ} \mathrm{C}$ for 20 minutes \\
\hline 5. Chemical & Algae with $2 \% \mathrm{H}_{2} \mathrm{SO}_{4}$ \\
\hline 6. Chemical + Thermal & Algae with $2 \% \mathrm{H}_{2} \mathrm{SO}_{4}$ subject to $121^{\circ} \mathrm{C}$ for 20 minutes \\
\hline 7. Enzymatic & Algae with cellulase and $\beta$-glucosidase \\
\hline 8. Enzymatic + Thermal & Algae with cellulase and $\beta$-glucosidase subject to $121^{\circ} \mathrm{C}$ for 20 minutes \\
\hline $\begin{array}{l}\text { 9. Enzymatic }+ \\
\text { Chemical }\end{array}$ & Algae with cellulase and $\beta$-glucosidase suspended in $1 \% \mathrm{H}_{2} \mathrm{SO}_{4}$ \\
\hline $\begin{array}{l}\text { 10. } \text { Enzymatic + } \\
\text { Chemical + Thermal }\end{array}$ & $\begin{array}{l}\text { Algae with cellulase and } \beta \text {-glucosidase suspended in } 1 \% \mathrm{H}_{2} \mathrm{SO}_{4} \text { subject } \\
\text { to } 121^{\circ} \mathrm{C} \text { for } 20 \text { minutes }\end{array}$ \\
\hline
\end{tabular}




\subsubsection{Biobutanol Production in Batch using Algal Biomass}

Following the hydrolysis treatments, the algal biomass was subjected to a fermentation process to produce biobutanol. Three different hydrolysis treatment options were applied and analyzed for the fermentation process for each of the two fusants; $\mathrm{CaCt}$ and $\mathrm{CbCt}$. In addition to this, parent strains of $C a, C b$, and $C t$ were also applied and analyzed for the fermentation process. The three different hydrolysis treatments included: 1) $\mathrm{H}_{2} \mathrm{O}$ with cellulase and $\beta$ glucosidase 2) $1 \% \mathrm{H}_{2} \mathrm{SO}_{4}$ and 3) $1 \% \mathrm{H}_{2} \mathrm{SO}_{4}$ with cellulase and $\beta$--glucosidase. A total of 2 grams of algae was utilized in each fermentation process. Fermentation experiments using enzymatically pretreated algae are considered as separate hydrolysis and fermentation (SHF). Moreover, fermentation experiments without enzymes in the pre-treatment of the algal biomass are considered SSF. Different fermentation procedures were applied in this study due to the variation in optimal temperatures required for efficient enzymatic activity and fermentation. The optimal temperature for cellulase and $\beta$-glucosidase activity was $40{ }^{\circ} \mathrm{C}$ whereas the optimal temperature for fermentation was $45^{\circ} \mathrm{C}$. Thus, fermentation experiments using enzymes for saccharification carried out hydrolysis treatments separately. In both fermentation procedures, the samples were inoculated with the mutated fusant exposed to UV radiation (described in section 3.3.2) showing highest cell count in the presence of $15 \mathrm{~g} / \mathrm{L}$ of biobutanol. The parent strains of Clostridia were utilized in the fermentation process without any further mutagenesis. A total of 9 fermentation samples were examined. The table below lists the details of each fermentation sample. A sample from each fermentation jar was taken initially and then every 24 hours for up to 120 hours. The fermentation samples for each day were stored in a $-82^{\circ} \mathrm{C}$ freezer until further analysis. Furthermore, the samples were later analyzed using High Performance Liquid Chromatography (HPLC) to examine the yield of biobutanol, sugars, and other solvents. 
Table 3.2 Fermentation samples

\begin{tabular}{|l|l|l|}
\hline Experiment & Bacteria & Pre-Treatment \\
\hline $\mathbf{1}$ & $\mathrm{CaCt}$ & $\mathrm{H}_{2} \mathrm{O}+$ cellulase and $\beta$-glucosidase \\
\hline $\mathbf{2}$ & $\mathrm{CaCt}$ & $1 \% \mathrm{H}_{2} \mathrm{SO}_{4}$ \\
\hline $\mathbf{3}$ & $\mathrm{CaCt}$ & $1 \% \mathrm{H}_{2} \mathrm{SO}_{4}+$ cellulase and $\beta$-glucosidase \\
\hline $\mathbf{4}$ & $\mathrm{CbCt}$ & $\mathrm{H}_{2} \mathrm{O}+$ cellulase and $\beta$-glucosidase \\
\hline $\mathbf{5}$ & $\mathrm{CbCt}$ & $1 \% \mathrm{H}_{2} \mathrm{SO}_{4}$ \\
\hline $\mathbf{6}$ & $\mathrm{CbCt}$ & $1 \% \mathrm{H}_{2} \mathrm{SO}_{4}+$ cellulase and $\beta$-glucosidase \\
\hline $\mathbf{7}$ & $\mathrm{Ca}$ & $1 \% \mathrm{H}_{2} \mathrm{SO}_{4}+$ cellulase and $\beta$-glucosidase \\
\hline $\mathbf{8}$ & $\mathrm{Cb}$ & $1 \% \mathrm{H}_{2} \mathrm{SO}_{4}+$ cellulase and $\beta$-glucosidase \\
\hline $\mathbf{9}$ & $\mathrm{Ct}$ & $1 \% \mathrm{H}_{2} \mathrm{SO}_{4}+$ cellulase and $\beta$-glucosidase \\
\hline
\end{tabular}

\subsubsection{Algal Oil Extraction}

In the present study, oil extraction was examined from virgin algae and from the remaining algae recovered after the fermentation process through two primary methods that both utilized green solvents. Ultrasonication and a modified, green Bligh and Dyer method, adopted from Yang et al. (2012), were applied to the algal biomass to extract oil. Furthermore, these two methods were analyzed and compared as well as combined in a two-step extraction process. Ultimately, this was to determine the potential of applying ultrasonication to the algae cells and then using a modified, green Bligh and Dryer method afterwards. Furthermore, this study also investigated oil extraction following fermentation. In this investigation, the same dry weight algae used in a fermentation experiment was exposed to the optimal conditions that resulted in the highest oil yield in the previous oil extraction experiments, which utilized virgin algal 
biomass. Parameters of this specific experiment were used to determine the impact of using virgin versus using the remaining algae recovered following fermentation on the overall yield.

Table 3.3 represents the oil extraction experiments and their associated parameters.

Table 3.3 Oil extraction experiments

\begin{tabular}{|c|c|c|}
\hline Experiment & Oil Extraction Method & Details \\
\hline 1 & Ultrasonication & $\begin{array}{l}\text { Time }=10 \text { minutes; } 2 \mathrm{~g} \text { ground algae } ; 50 \mathrm{~mL} \\
\text { limonene }\end{array}$ \\
\hline 2 & Ultrasonication & Time $=20$ minutes $; 2 \mathrm{~g}$ ground algae; $50 \mathrm{~mL}$ limonene \\
\hline 3 & Ultrasonication & Time $=30$ minutes $; 2 \mathrm{~g}$ ground algae; $50 \mathrm{~mL}$ limonene \\
\hline 4 & Ultrasonication & Time $=40$ minutes $; 2 \mathrm{~g}$ ground algae; $50 \mathrm{~mL}$ limonene \\
\hline 5 & Ultrasonication & Time $=10$ minutes; 2 ground algae; $50 \mathrm{~mL}$ p-cymene \\
\hline 6 & Ultrasonication & Time $=20$ minutes $; 2$ ground algae; $50 \mathrm{~mL}$ p-cymene \\
\hline 7 & Ultrasonication & Time $=30$ minutes; 2 ground algae; $50 \mathrm{~mL}$ p-cymene \\
\hline 8 & Ultrasonication & Time $=40$ minutes; 2 ground algae; $50 \mathrm{~mL}$ p-cymene \\
\hline 9 & Ultrasonication & $\begin{array}{l}\text { Control experiment; } 2 \mathrm{~g} \text { ground algae; } 50 \mathrm{~mL} \text { water } \\
\text { Time }=40 \text { minutes }\end{array}$ \\
\hline 10 & Modified, green Bligh and Dyer & Direct solvent (d-limonene) \\
\hline 11 & Modified, green Bligh and Dyer & Direct solvent (p-cymene) \\
\hline 12 & Combination method & $\begin{array}{l}\text { Ultrasonication Time }=30 \text { minutes in } 50 \mathrm{~mL} \mathrm{~d}- \\
\text { limonene followed by direct solvent method }\end{array}$ \\
\hline 13 & Combination method & $\begin{array}{l}\text { Ultrasonication Time }=30 \text { minutes in } 50 \mathrm{~mL} \\
\mathrm{p}=\text { cymene followed by direct solvent method }\end{array}$ \\
\hline
\end{tabular}

Ultrasonication of the algal biomass was carried out using the UP200Ht Handheld Ultrasonic Homogenizer. First, approximately $50 \mathrm{~mL}$ of the terpene (either d- limonene or pcymene) was used with approximately 2 grams of ground algae. Next, the ultrasonic homogenizer was set to standard parameters of max frequency $26 \mathrm{kHz}, 100 \%$ amplitude and 200 W of power. Each solvent was exposed to 4 different times of 10 minutes, 20 minutes, 30 
minutes, and 40 minutes. A control sample was used with distilled $\mathrm{H}_{2} \mathrm{O}$ in place of the solvent and had an exposure time of 40 minutes. Each experiment was repeated to ensure validity.

The modified, green Bligh and Dyer method also used d-limonene and p-cymene to extract oil from the algal biomass. A solution of terpene with water created the polar and nonpolar solvent ratio necessary for oil separation. First, 2 grams of ground algae was added to approximately $30 \mathrm{~mL}$ of water and heated for 30 minutes at $30^{\circ} \mathrm{C}$ (Yang et al., 2012). Once heated, the suspension was centrifuged at 1,000 g for 10 minutes. Following centrifuging, the algae debris was removed, and the remaining suspension was added to a $50 \mathrm{~mL}$ test tube. Additional distilled water was added to the $45 \mathrm{~mL}$ mark and $5 \mathrm{~mL}$ of d-limonene was added to fill the entire test tube, reaching a total volume of $50 \mathrm{~mL}$ (Yang et al., 2012). This process was repeated with the addition of the second solvent, p-cymene. Test tubes were allowed to sit for approximately 1-2 hours. With the variation in density, the oil and solvent settled at the top while the water remained at the bottom of the test tube. Each experiment was repeated to ensure validity.

Following the individual experiments of ultrasonication and the modified, green Bligh and Dyer method, this study also examined the effect on oil yield when the two methods were combined into a two-step process. In the first step of this process, the algal biomass was subjected to ultrasonication followed by the modified, green Bligh and Dryer method. Ultrasonication parameters remained the same as described above. The time for sonication was set to 30 minutes and the algae was suspended in $50 \mathrm{~mL}$ distilled water. Following this, the algae suspension was centrifuged, and the algae debris was removed. The remaining solution was then transferred to a $50 \mathrm{~mL}$ test tube. With the sonication experiment only having a polar solvent, the non-polar terpene was added to the test tube. The solution was allowed to sit for approximately 
1-2 hours. Within this time, the oil and solvent settled at the top while the water remained at the bottom of the test tube. This process was repeated for the second terpene. Each experiment was repeated to ensure validity.

After each experiment, d-limonene and p-cymene needed to be separated and removed from the oil. A rotary evaporator was used in this instance to remove the solvent. A rotary evaporator is a machine that is commonly used to remove solvents from solutions in a gentle and efficient manner. The machine works as a continuous distillation apparatus that operates under vacuum conditions (Wang et al., 2018). With an air vacuum attached at the top, the rotary evaporator is able to increase the rate of evaporation as the pressure is reduced. Furthermore, a heating mantle assisted in heating the solution to the appropriate temperature for evaporation to begin. The vaporized solution travels through the apparatus to the top where it begins to condense as a result of the attached condenser. Once the solvent vapor begins to condense it changes phase and drips down in a liquid state. Upon solvent removal, the remaining suspension was solvent free. The amount of oil following each rotary evaporation was measured out. The remaining solution was boiled at the respective solvent boiling point to ensure that all solvent was removed. The boiling point and thus evaporation of oil is significantly higher than that of both limonene and cymene therefore oil was not lost during this process.

\subsubsection{Algal Oil Extraction Post-Fermentation}

Following fermentation, the algal debris was recovered from the fermentation broth. Next, the fermentation sample was transferred to a test tube and centrifuged at $1,000 \mathrm{~g}$ for 10 minutes. Following this, the algal debris was filtered out and collected for drying prior to oil extraction. The algal debris was then exposed to water steam to facilitate drying of the biomass. Next, the algal debris was then placed in an oven for 2 hours at a temperature of $60^{\circ} \mathrm{C}$. 
Following the removal and drying of the algal biomass post-fermentation, the biomass was subjected to the two-step oil extraction process as described above. The algal biomass was exposed to the optimal conditions of ultrasonication (30 minutes, in distilled water) followed by centrifugation and algae debris removal. After this, d-limonene was then added to the remaining suspension and allowed to sit for 1-2 hours. Next, the solvent was removed as described in section 3.2.4. The oil yield was calculated as stated in section 3. 3.5.

\subsection{Analytical Procedures}

\subsubsection{High-Performance Liquid Chromatography (HPLC)}

The sugar and solvent composition were analyzed using pre-calibrated HPLC. The equipment (Agilent 1260) incorporated an ion exchange pump, a pump series (Agilent 1290 Infinity II), an auto sampler (Agilent 1290 Infinity II) and refractive index detector (RID) (Agilent 1260 Infinity II). Two different columns were used during the HPLC analysis. The HPLC column used to detect sugar concentration was the Shodex SP010. The HPLC column used to detect solvent and acid concentrations was the [Aminex HPX-87H]. Each sample was initially centrifuged at 4,500 at 25 minutes and double filtered through $0.2 \mu \mathrm{m}$ filters (Whatman, USA). A total of $10 \mu \mathrm{L}$ from each dilute sample was injected into the column and circulated for 35 minutes at a flow rate of $0.6 \mathrm{~mL} / \mathrm{min}$. The column temperature was maintained at $80^{\circ} \mathrm{C}$ using the column heater CH-30 controlled by Eppendorf TC 50 (Buday et al., 1990). Data was processed using the OpenLab CDS Chemstation software. It was necessary to ensure that HPLC vials were filled adequately to ensure minimal headspace to reduce solvent loss in the vapor phase. Sugar concentrations and ABE concentrations were quantified from calibration curves that were developed from standard solutions of known concentrations; Appendix C. 


\subsubsection{Cell Count and Growth Rate Calculations}

Following UV mutagenesis, the cell concentration in the culture, taken at 5-minute intervals for a total time of 25 minutes was determined using a hemocytometer (Bright-Line, Hausser Scientific). The hemocytometer was cleaned thoroughly with alcohol prior to each sample. Each sample culture was diluted to a factor of $10^{5} .0 .1 \mathrm{~mL}$ of the cell culture was added to $0.9 \mathrm{~mL}$ of autoclaved distilled water. Thus, creating a dilution factor of 10 . Subsequently, this process was repeated 5 times to ensure a sample that was diluted to a factor of $10^{5}$. Approximately $0.5 \mathrm{~mL}$ of diluted cell suspension was then removed and applied on the hemocytometer. Using a microscope (Nikon Eclipse 55i), the grid lines on the hemocytometer were focused with a 10x objective. Cell count was carried out by determining the number of cells in each of the four large squares. Following this, the average number of cells was calculated. The average number of cells was multiplied by $10,0000\left(10^{5}\right)$ to account for the dilution factor.

The above method was also used to calculate the surviving fraction (SF) which is the number of cells that survived the exposure to the UV radiation. In addition to this, the relative induced mutation frequency $(\mathrm{RF})$ calculated as a proportion of the mutant strain in the cell population that had survived exposure to the UV radiation divided by the proportion of the mutant strain that was present but not exposed to the UV radiation (Bowring and Morris, 1986).

\subsubsection{Biomass Composition Analysis}

Cellulose and hemicellulose contents of the algal biomass were quantified as per the National Renewable Energy Laboratory (NREL) as described by Ververis et al. (2007). Four samples of air-dried algal biomass, weighing 2 grams each (ground to $75 \mu \mathrm{m}$ ) were boiled with $10 \mathrm{~mL} 72 \%$ w/w $\mathrm{H}_{2} \mathrm{SO}_{4}$ solution for approximately 4.5 hours. The purpose of this was to hydrolyze the cellulose and hemicellulose. The mixture following boiling was filtered through a crucible and 
the solid reside was dried at $105^{\circ} \mathrm{C}$ for approximately 24 hours and weighed (W1). The residue was then placed in a pre-dried crucible and heated at $600^{\circ} \mathrm{C}$ for approximately 5 hours. Upon cooling, the residue was weighed (W2) and the ash content (\%) was determined as described below. The glucose (C1) and reducing sugar (C2) was determined through HPLC. Subsequently, the cellulose and hemicellulose content were determined as follows:

$$
\text { Cellulose content }\left(\% \frac{w}{w}\right)=\left(\frac{0.9}{0.96}\right) X C 1 X\left(\frac{V}{m}\right) X \alpha X 100
$$

where 0.9 is the glucose conversion factor that is derived from the molecular weight ratio of the polymer and the monomer of hexose. The saccharification yield was determined to be $0.96 . \mathrm{C} 1$ is the glucose concentration as determined through HPLC in $\mathrm{g} / \mathrm{L}$. V is the total volume of the sugar expressed in $\mathrm{g} / \mathrm{L}$ and $\mathrm{M}$ is the dry weight of the algal biomass expressed in grams. Finally, $\alpha$ is the dilution of the sample (if any).

$$
\text { Hemicellulose content }\left(\% \frac{w}{w}\right)=\left(\frac{0.88}{0.93}\right) X(C 2-C 1) X\left(\frac{V}{M}\right) X \alpha X 100
$$

where 0.88 is the coefficient that is derived from the molecular weight ratio of the polymer and the monomer pentose. 0.93 is the saccharification yield of xylene and xylose. $\mathrm{C} 2$ is the total reducing sugars (TRS) expressed in $\mathrm{g} / \mathrm{L}$ as determined through HPLC. V is the total volume of the sugar expressed in $\mathrm{g} / \mathrm{L}$ and $\mathrm{M}$ is the dry weight of the algal biomass expressed in grams. Finally, $\alpha$ is the dilution of the sample (if any).

\subsubsection{Cell Disruption}

An important evaluation of the oil extraction methods carried out in this study is to determine the cell disruption efficiency. The cell disruption efficiency will indicate how effective the extraction method is relative to the initial cell concentration prior to an extraction procedure. Furthermore, the cell disruption efficiency provides a value to how effective the oil extraction 
method is in terms of rupturing the microalgae cells. It is calculated by the equation below as indicated by Natarajan et al. (2014):

$$
n=\frac{\left(C_{O}-C_{f}\right)}{C_{O}} \times 100
$$

Where, $C_{o}$ is the initial cell count before the oil extraction method and $C_{f}$ is the final cell count following the oil extraction method. The cell concentration was determined as indicated in section 3.3.2.

\subsubsection{Oil Yield}

In the present study, oil quantification was carried out following the use of the rotary evaporator to remove the terpene solvent. The oil sample was removed using a spatula and placed in a pre-weighed Eppendorf tube $\left(\mathrm{W}_{1}\right)$ (Prabrakan and Ravindran, 2011). The Eppendorf tube containing the oil was weighed on an analytical scale $\left(\mathrm{W}_{2}\right)$ (Prabrakan and Ravindran, 2011). The final weight of the oil ( $\left.\mathrm{W}_{\mathrm{O}}\right)$ was determined by the equation below.

$$
W_{O}=W_{2}-W_{1}
$$

Based upon the weight of the oil, the oil yield was calculated as per the total amount of lipid content in the algae. The following equation was used to determine the oil yield:

$$
\text { Oil Yield } \%=\left(\frac{W_{o}}{W_{A O}}\right) \times 100
$$

Where, $\mathrm{W}_{\mathrm{O}}$ is the weight of the oil following oil extraction and solvent removal and $\mathrm{W}_{\mathrm{AO}}$ is the actual weight of lipid in the algal biomass. 


\subsubsection{Error Analysis}

A critical aspect of data analysis is to calculate the error and to determine the repeatability of the results. This study repeated experiments and determined the associated error. The fermentation and oil extraction experiments were carried out in either duplicates or triplicates to determine the standard deviation and relative standard deviation (RSD). The following equation was used to calculation standard deviation: Standard deviation (RSD) was calculated using the following equation:

$$
\sigma=\sqrt{\frac{\sum(x-\vec{x})^{2}}{(n-1)}}
$$

Relative standard deviation was calculated using the following equation:

$$
R S D=\left(\frac{\sigma}{\mu}\right) \times 100
$$

Where $\sigma$ is standard deviation and $\mu$ is the mean.

Appendix D and E indicate the raw data for the fermentation and oil extraction procedures. Tables D1-D2 and Tables E1-E4 indicate the error calculations for all the fermentation experiments and oil extraction experiments. The error (RSD) is below $1 \%$ for all experiments and within the acceptable range of error. The standard deviation of biobutanol produced from algae was within the range of 0.01 to 0.04 and percent error within the range of $0.05 \%$ to $0.64 \%$. The standard deviation of ultrasonication experiments was within the range of 0.001 and 0.02 and percent error within the range of $0.05 \%$ to $0.35 \%$. The standard deviation of the modified, Bligh and Dyer method was within the range of 0.005 to 0.0025 and percent error within the range of $0.04 \%$ to $0.07 \%$. The standard deviation from the combined extraction 
process using virgin algal biomass was 0.01 and percent error within the range of $0.04 \%$ to $0.05 \%$. The standard deviation from the combined extraction process using the recovered algal biomass post-fermentation was 0.01 and the percent error was. $0.04 \%$. 


\section{CHAPTER 4: \\ RESULTS AND DISCUSSION}

\subsection{Fermentation and Biobutanol Production}

\subsubsection{UV Mutagenesis}

The fusants of $\mathrm{CaCt}$ and $\mathrm{CbCt}$ were subjected to $\mathrm{UV}$ mutagenesis as described earlier. The goal of mutagenesis is to produce mutant genes of a strain of a biological organism. Furthermore, the mutation has the potential to enhance or perhaps produce novel, desirable functions. UV radiation was applied to each of the fusants for different times to determine the effect upon cell count. Appendix B indicate shows the raw data of the UV mutagenesis experiments carried out upon $\mathrm{CaCt}$ and $\mathrm{CbCt}$. Figure 4.1 illustrates the cell count after UV mutagenesis of fusants $\mathrm{CaCt}$ and $\mathrm{CbCt}$ following different time intervals. Figure 4.2 illustrates the cell count after UV mutagenesis of fusants $\mathrm{CaCt}$ and $\mathrm{CbCt}$ in the presence of $15 \mathrm{~g} / \mathrm{L}$ of butanol. Fusants, exposed to UV mutagenesis, were grown in the presence of butanol as means for a toxicity test. According to Figure 4.1, when subjected to UV radiation the fused strain of $\mathrm{CaCt}$ began with a cell concentration of $26 \times 10^{5}$ cells $/ \mathrm{mL}$ and began to experience a decline in cell concentration following each 5-minute interval. Following the 25-minute exposure, the cell concentration dropped to $0.5 \times 10^{5}$ cells $/ \mathrm{mL}$. When examining the effect of UV mutagenesis upon $\mathrm{CbCt}$, it can be seen that when subjected to UV radiation the fused strain began with an initial cell concentration of $28.75 \times 10^{5}$ cells $/ \mathrm{mL}$. Similar to $\mathrm{CaCt}$, the fusant $\mathrm{CbCt}$ also experienced a decline in cell concentration following each 5-minute interval. After the 25-minute exposure to UV radiation, the cell concentration of $C b C t$ dropped to $1.0 \times 10^{5}$ cells $/ \mathrm{mL}$. Prolonged UV exposure has a positive correlation with cell count due to the impact of excessive DNA damage (Ikehata and Ono, 2011). According to Figure 4.2, when exposed to UV radiation in the presence of $15 \mathrm{~g} / \mathrm{L}$ of butanol, the initial concentration for $C a C t$ was $1.5 \times 10^{5}$ cells $/ \mathrm{mL}$. 
Unlike the pattern depicted in Figure 4.1 for $\mathrm{CaCt}$, in the presence of butanol, the cell concentration experienced an increased until the 10-minute exposure time. Following, the 10minute exposure time a decline in cell concentration can be seen. Thus, indicating the highest resistance to $15 \mathrm{~g} / \mathrm{L}$ of butanol at an exposure time of 10 minutes. The cell concentration for $\mathrm{CaCt}$, with exposure to UV radiation in butanol, experienced an increased from $1.5 \times 10^{5}$ cells $/ \mathrm{mL}$ (time=0) to $11.25 \times 10^{5}$ cells $/ \mathrm{mL}$ (time=10). Following the 10-minute exposure, the cell concentration of $\mathrm{CaCt}$ experienced a decline to $9.5 \times 10^{5}$ cells $/ \mathrm{mL}$ after the 15 -minute exposure and eventually a cell concentration of $0.25 \times 10^{5}$ cells $/ \mathrm{mL}$ after the final 25 -minute exposure. A similar pattern can be seen for the fused strain of $C b C t$ in Figure 4.2. Prior to UV radiation exposure, the cell concentration of $\mathrm{CbCt}$ in the presence of $15 \mathrm{~g} / \mathrm{L}$ butanol, was $2.25 \times 10^{5}$ cells $/ \mathrm{mL}$. After the first 5-minute interval, the cell concentration of $\mathrm{CbCt}$ experienced a drastic increase to $12 \times 10^{5} \mathrm{cells} / \mathrm{mL}$. Similar to $\mathrm{CaCt}$, the cell concentration of $\mathrm{CbCt}$ also experienced an increased until the 10-minute exposure time. Following, the 10-minute exposure, the cell concentration of $\mathrm{CbCt}$ also experienced a decline to $10.5 \times 10^{5}$ cells $/ \mathrm{mL}$. Subsequently, the cell concentration declined down to $0.25 \times 10^{5}$ cells $/ \mathrm{mL}$ after the 25 -minute exposure. The highest resistance to $15 \mathrm{~g} / \mathrm{L}$ of butanol was at 10 minutes of exposure time. 


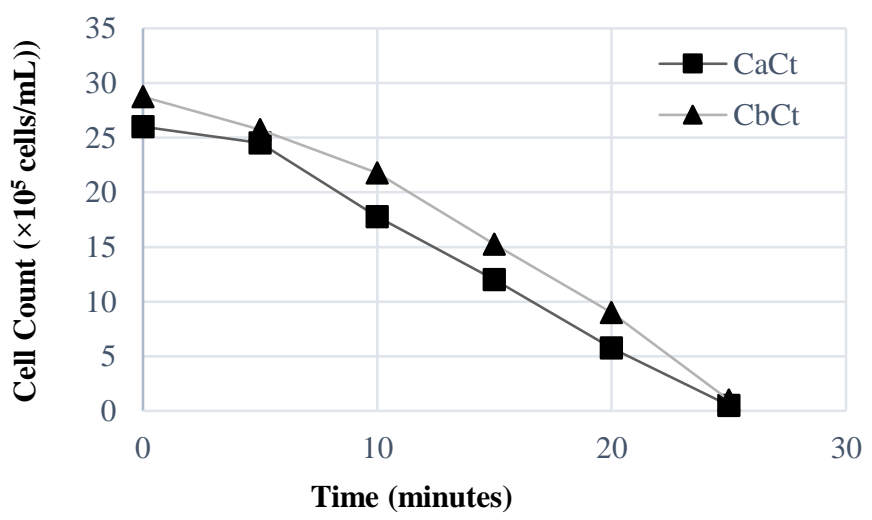

Figure 4.1 Change in cell concentration following $\mathrm{UV}$ mutagenesis for fused strains $\mathrm{CaCt}$ and $\mathrm{CbCt}$ in the absence of butanol.

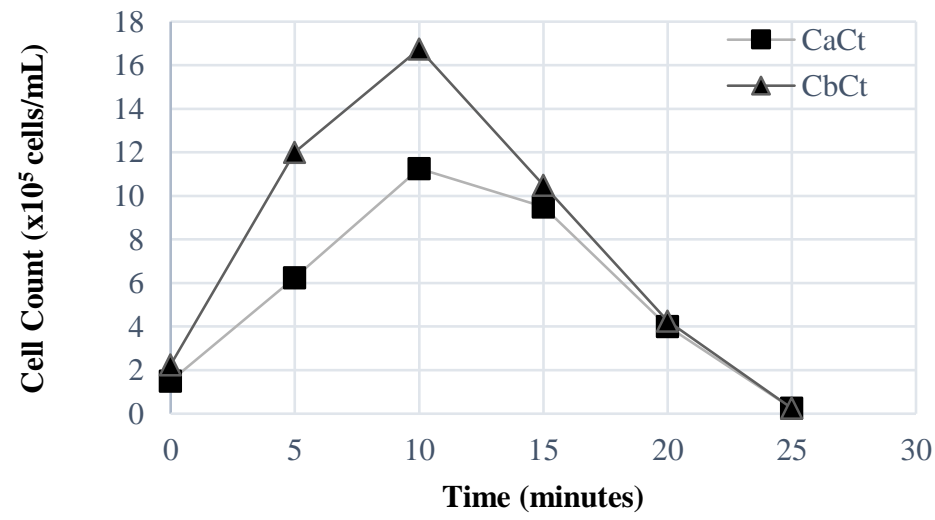

Figure 4.2 Change in cell concentration following $\mathrm{UV}$ mutagenesis for fused bacteria $\mathrm{CaCt}$ and $\mathrm{CbCt}$ in the presence of butanol

Figures 4.3 indicates the surviving fraction and relative induced mutation frequency of $\mathrm{CaCt}$ and $\mathrm{CbCt}$, exposed to UV mutagenesis. As illustrated in the figures, the surviving fraction decreased over the exposure time. Furthermore, following the 15-minute exposure time the surviving fraction began to decrease significantly for both fusants indicating excessive DNA damage ((Ikehata and Ono, 2011). It is apparent that prolonged exposure to UV radiation implicated both fusants in a similar manner as the surviving fraction curves have similar shapes. However, it can be concluded that $C b C t$ resulted in a higher surviving fraction following the full- 
time exposure of UV radiation. The surviving fraction after the 25-minute exposure was 0.019 (equivalent of $0.5 \times 10^{5}$ cells $/ \mathrm{mL}$ ) and 0.035 (equivalent of $1 \times 0^{5}$ cells $/ \mathrm{mL}$ ) for $C a C t$ and $C b C t$; respectively. Therefore, this indicates the stronger tolerance of UV radiation for $\mathrm{CbCt}$. However, in the present study both fusants were used in the SSF of algal biomass. The mutant strains showing the highest relative frequencies and tolerance butanol for use for the SSF procedures.

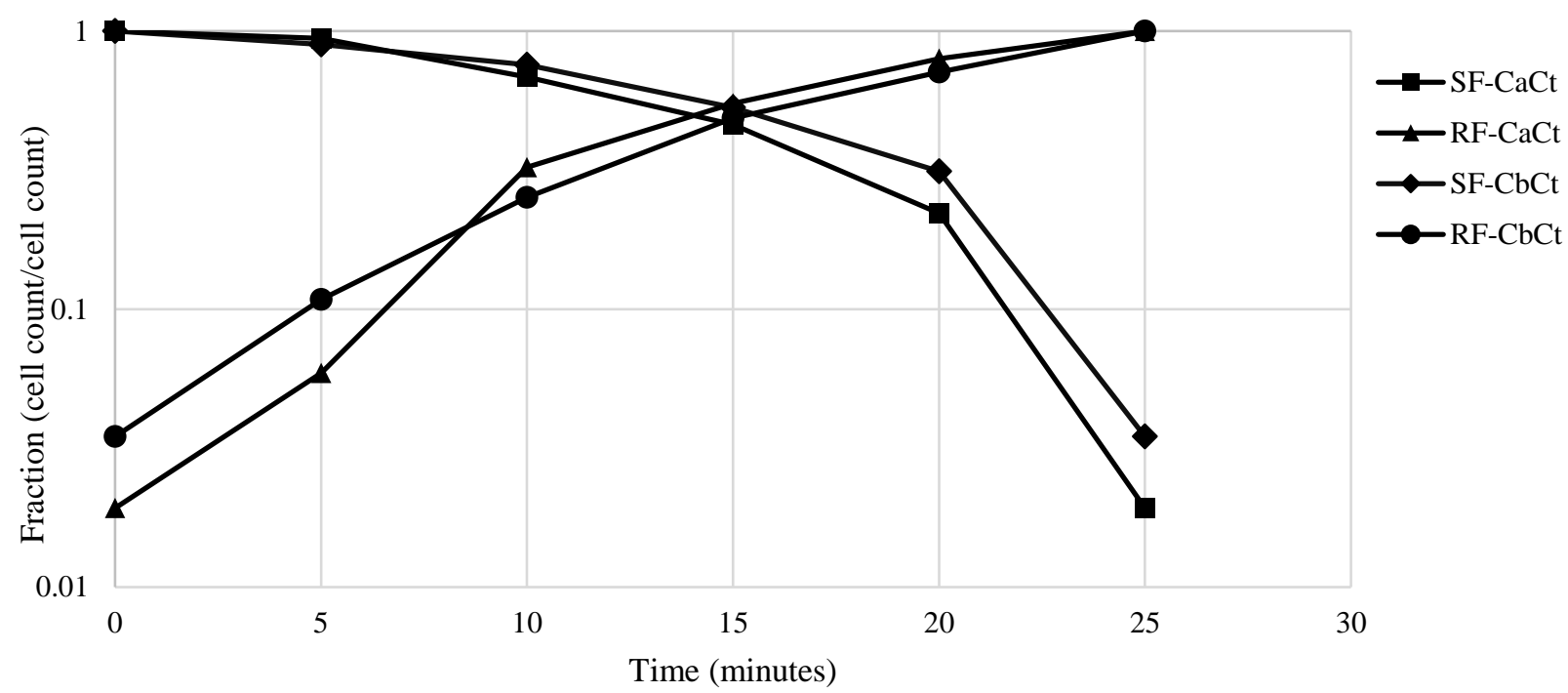

Figure 4.3 Surviving fraction (SF) and relative frequency (RF) of induced mutation from UV mutagenesis of fusants $\mathrm{CaCt}$ and $\mathrm{CbCt}$

\subsubsection{Algae Pre-Treatment}

Table 4.1 indicates the individual and total sugar concentrations from algal biomass that were subject to different pre-treatment options. The hydrolysis of algae through the designated pre-treatments indicates that treatment \#8 was the most successful in breaking down the algae structure and releasing sugars. Table 4.1 indicates that treatment \#8 resulted in the highest TRS of $27.78 \mathrm{~g} / \mathrm{L}$. The hydrolysis of treatment \#8 consisted of chemical, enzymatic and thermal. The second highest TRS resulted from treatment \#7 which was comprised of the same pre-treatment options as treatment \#8 excluding the thermal treatment. Therefore, the corresponding TRS value 
is merely $2 \mathrm{~g} / \mathrm{L}$ less. Furthermore, this can also indicate that the thermal treatment had a positive effect on the extraction of carbohydrates and subsequent sugar release. Total sugar concentrations in the hydrolysate generally had higher values when exposed to the thermal pretreatment when compared to those treatment samples where heat was not applied. Table 4.1 indicates this as each subsequent treatment is slightly higher than the previous. For example, treatment \#2 possessed the same components with the addition of the thermal pre-treatment. However, it is important to note that following these treatments, treatment \#6 was most successful in breaking down the algal structure and releasing sugar. The hydrolysis treatment of \#6 relied only upon water and enzymes coupled with a thermal pre-treatment. Subsequently, this data revealed that water and enzymes coupled with a thermal treatment can be rather efficient in breaking down the algal structure.

Further examination can specify that treatments \#3 and \#4 resulted in significantly higher TRS values when compared with treatments \#9 and \#10. This indicated that $1 \%$ acid treatment is far more efficient than $2 \%$ acid treatment (Kumar et al., 2013). This can be attributed to the less rigid structure of algae, which happens to be partially oxidized upon treatment with higher concentrations of sulfuric acid (Nguyen et al., 2009). Furthermore, with the highest TRS values attributed to treatment options comprised of enzymes (treatments \#6, \#7, \#8) it can be concluded that enzymatic pre-treatment is an integral component for the disintegration of the cellulosic structure and increasing the bioavailability of sugar monomers. Thus, this further indicates the significance of enzymes in breaking down cellulose and hemicellulose. Furthermore, it can also be concluded that hydrolysis treatments did in fact not require the addition of xylanase to produce xylose. Xylanase is an enzyme used to break down xylan from hemicellulose to xylose. 
It has been indicated that cellulase is sufficient enough to break down hemicellulose into xylose (Qing et al., 2010).

Table 4.1 Sugar Concentrations for different pre-treatment options for algal biomass

\begin{tabular}{|c|c|c|c|c|c|c|c|}
\hline $\begin{array}{l}\text { Hydrolysis } \\
\text { Treatment }\end{array}$ & Detail & $\begin{array}{l}\text { Glucose } \\
\text { (g/L) }\end{array}$ & $\begin{array}{l}\text { Xylose } \\
(\mathrm{g} / \mathrm{L})\end{array}$ & $\begin{array}{l}\text { Galactose } \\
\text { (g/L) }\end{array}$ & $\begin{array}{l}\text { Mannose } \\
\text { (g/L) }\end{array}$ & $\begin{array}{l}\text { Arabinose } \\
\text { (g/L) }\end{array}$ & $\begin{array}{l}\text { Total } \\
\text { (g/L) }\end{array}$ \\
\hline 1 & $\mathrm{H}_{2} \mathrm{O}$ & 4.12 & 2.00 & 1.12 & 1.90 & 1.56 & 10.71 \\
\hline 2 & $\mathrm{H}_{2} \mathrm{O}^{*}$ & 4.41 & 2.02 & 1.20 & 2.00 & 1.60 & 11.22 \\
\hline 3 & $1 \% \mathrm{H}_{2} \mathrm{SO}_{4}$ & 8.01 & 4.73 & 1.57 & 4.01 & 1.70 & 20.02 \\
\hline 4 & $1 \% \mathrm{H}_{2} \mathrm{~S}_{4} *$ & 8.96 & 4.86 & 1.64 & 4.06 & 1.72 & 21.24 \\
\hline 5 & EZ-1 & 8.01 & 5.41 & 1.72 & 4.05 & 1.76 & 20.96 \\
\hline 6 & EZ-2 & 8.60 & 5.99 & 1.76 & 4.27 & 2.68 & 23.30 \\
\hline 7 & EZ-3 & 11.04 & 5.82 & 1.84 & 4.35 & 2.72 & 25.78 \\
\hline 8 & EZ-4 & 11.97 & 6.63 & 1.92 & 4.46 & 2.81 & 27.78 \\
\hline 9 & $2 \% \mathrm{H}_{2} \mathrm{SO}_{4}$ & 3.96 & 1.96 & 1.05 & 1.98 & 1.57 & 10.51 \\
\hline 10 & $2 \% \mathrm{H}_{2} \mathrm{SO}_{4} *$ & 3.99 & 1.98 & 1.08 & 2.04 & 1.59 & 10.69 \\
\hline
\end{tabular}

As depicted in Table 4.2, when compared with the other four agricultural resides, algae possess lower cellulose and hemicellulose contents which can directly correlate with its sugar potential. Compared with wheat straw, hemp, sugar cane, and switch grass, the algal biomass investigated in this study possess a TRS of $27.78 \mathrm{~g} / \mathrm{L}$ subject to chemical, enzymatic, and thermal hydrolysis treatments. The four other agricultural resides, used as a point of comparison, have also been subject to similar hydrolysis treatments. It can be indicated that wheat straw possesses a significantly higher TRS potential of $60.2 \mathrm{~g} / \mathrm{L}$ when compared with algae. This can 
be directly correlated with the cellulose and hemicellulose contents. Wheat straw on average contains $35-39 \%$ cellulose whereas the algae investigated in this report contained only $16 \%$. Cellulose is directly converted to glucose which is the primary monosaccharide comprised in biomass and moreover, significantly contributes to the overall TRS. Hemp, sugarcane, and switch grass also possess higher rates of cellulose which explain why their TRS values are also higher than that of the algae. With more than less than half of the cellulose content typically found in wheat straw and other residues, it is justified that algae possess lower glucose and subsequently lower TRS values.

Hemicellulose is another important physical component of biomass which contributes to the overall TRS value. As it can be seen from Table 4.2, the algae in question contains much lower hemicellulose values when compared with wheat straw, hemp, sugarcane, and switch grass. Through hydrolysis treatment, hemicellulose breaks down to different monosaccharides including xylose, mannose, galactose, and arabinose which in addition to the glucose, yield the TRS potential. Therefore, this further explains why the TRS of algae is lower. However, another important physical component of biomass, as previously discussed is the lignin content. Unlike the other four agricultural residues, algae contain little to low lignin and in this investigation, the Stichococcus species of algae does not possess lignin. Therefore, this significantly eliminates the recalcitrant component and ensuring the relatively easier disintegration of cellulose and hemicellulose to produce simple sugars necessary for fermentation. Furthermore, this can also be correlated with much less rigorous treatment options. This can be particularly important when choosing a hydrolysis technique that is both energy efficient and environmentally friendly

Furthermore, as discussed, the crystalline nature of cellulose contributes to the overall recalcitrant nature of lignocellulosic material. Hemicellulose furthers this difficult characteristic 
because it acts as a physical barrier to the cellulose. Hemicellulose microfibers can be tightly bound and sheathed in a layer of pectin which subsequently act a strong physical barrier to the cellulose. Therefore, higher amounts of cellulose and hemicellulose, as with wheat straw, increase the recalcitrant nature and consequently require more energy for hydrolysis.

As previously discussed, the three components found in lignocellulosic material, each play an integral role in the overall structure, degradability, and total reducing sugar potential. The amount of cellulose, hemicellulose, and lignin found in a biomass can indicate its resistance to biodegradability and subsequent sugar release for bio-alcoholic fuel production. Although it has been indicated that higher amounts of cellulose and hemicellulose found in biomass can yield a greater TRS potential it can also indicate its increased recalcitrant nature towards hydrolysis. Wheat straw, hemp, sugar cane, and switch grass all possess higher values of cellulose and hemicellulose when compared with the algae investigated in this study. However, it is important to note that with the reduced values of cellulose and hemicellulose algae is relatively easier to breakdown. As indicated, water and enzymes can be quite efficient in breaking down the algal structure. Additionally, the specific strain investigated in this report contains no lignin. As previously discussed, lignin is the binding material which holds the cellulose and hemicellulose together. Furthermore, its irregular and complex structure make it highly resistant to chemical or enzymatic hydrolysis. Therefore, removing this component makes algal biomass hydrolysis considerably less rigorous. As previously discussed, algae have significantly higher growth rates when compared with second-generation feedstocks such as wheat straw. Considering this, algae can make up for their lower potential to produce sugars for fermentation as they have the capacity to grow at significantly higher rates and yield higher amounts of biomass. It is also important to note that algae are comprised of lipids which can also be extracted, in addition to 
the sugars, to produce other biofuels such as biodiesel. Based on values indicated by Burlew (1976), the carbohydrate and lipid contents of Stichococcus are 38.9\% and 38.5\%; respectively. Thus, this indicates that although the sugar potential of algae may be lower than wheat straw, for instance, it possesses lipids which are not found in second-generation biomass. Lipids can be extracted to produce other energy containing fuels such as bio-oil and biodiesel. Therefore, with the addition of lipids and the ability to produce other biofuels, it can be proclaimed that the energy availability of algae can be rather higher than another biomass.

Table 4.2 Lignocellulosic composition and TRS (g/L) of various agricultural residues

\begin{tabular}{|c|c|c|c|c|c|}
\hline Biomass & Cellulose \% & Hemicellulose \% & Lignin \% & $\begin{array}{l}\text { TRS } \\
(\mathrm{g} / \mathrm{L})\end{array}$ & Reference \\
\hline Algae & 16.25 & 12.62 & -- & 27.78 & Present study \\
\hline $\begin{array}{l}\text { Wheat } \\
\text { straw }\end{array}$ & $35-39$ & $23-30$ & $12-16$ & 60.2 & Qureshi et al., 2007 \\
\hline Hemp & $40-48$ & $18-24$ & $21-24$ & 34.9 & $\begin{array}{l}\text { Stevulova et al., } \\
2014 \\
\text { Abraham et al., } \\
2013\end{array}$ \\
\hline Sugarcane & $25-42$ & 25 & 20 & $60-70$ & Kumar et al., 2017 \\
\hline Switchgrass & $35-40$ & $25-30$ & $15-20$ & 31.1 & Singh et al., 2014 \\
\hline
\end{tabular}

Table 4.3 shows the higher heating values (HHV) of different agricultural crops. HHV can be defined as the amount of heat that is released unit of mass or volume of fuel once it has been combusted (Yin, 2011; Motghare et al., 2016; Tanger et al., 2013). It is an important indicator of the potential energy a specified product has. Analyzing and comparing the different HHV of agricultural crops can be extremely useful as it provides information regarding the energy potential. As previously discussed, despite the fact that microalgae feedstock has a lower potential to release sugars, when compared with other lignocellulosic biomass, it is however 
comprised of lipids. Lipids are another source of energy and add to the overall energy potential of algae. As indicated in the table below, when compared with other biomass, the microalgae used in the present study, has a greater HHV.

Table 4.3 Higher heating values of different agricultural crops

\begin{tabular}{|l|c|l|}
\hline Biomass & $\begin{array}{l}\text { Higher Heating Value } \\
(\mathbf{M J} / \mathbf{k g})\end{array}$ & Reference \\
\hline Wheat Straw & 18.94 & ECN (a), 2019 \\
\hline Hemp & 18.28 & ECN (b), 2019 \\
\hline Sugar cane & 16.91 & ECN (c), 2019 \\
\hline Switchgrass & 18.06 & ECN (d), 2019 \\
\hline $\begin{array}{l}\text { Stichococcus sp. } \\
\text { (microalgae) }\end{array}$ & 25.38 & Burlew, 1976, Demirbas, 2010 \\
\hline
\end{tabular}

As previously discussed, the lipid content of algae has the capacity to be converted to different biofuels thus increasing its energy potential. According to Burlew (1976), the carbohydrate and lipid content of the algal strain utilized in this investigation, is approximately equal. Considering this, it can be suggested that the additional carbohydrate content that would have normally been found in other lignocellulosic material such as wheat straw, is replaced by the lipid content. Therefore, algae make up for the reduced carbohydrate content through their lipid content which essentially is another form of potential energy.

\subsubsection{Simultaneous Saccharification and Fermentation and Separate Hydrolysis and Fermentation}

Biobutanol production from the algal biomass was achieved through a SSF and SHF process over the course of 120 hours equivalent to 5 days. Samples of each fermentation sample were taken every 24 hours. Each sample was analyzed for sugar, solvents and acids. Raw data 
for daily sugar, solvent and acid concentrations can be found in Appendix D. Table 4.4 shows the individual sugar concentrations for each of the nine fermentation experiments. Following fermentation, it can be seen that experiments \#3, 5 and 6 resulted in the lowest remaining sugar concentrations. Therefore, this indicates that experiments \#3, 5, and 6 were the most successful in releasing sugars necessary for ABE production. Experiment \#6 resulted in the least amount of total sugars thus indicating that fusant $C b C t$ with hydrolysis of algae (chemical, thermal, and enzymatic) was most successful in breaking down and utilizing majority of available sugars for ABE production.

Table 4.4 Individual sugar concentrations for each fermentation experiment

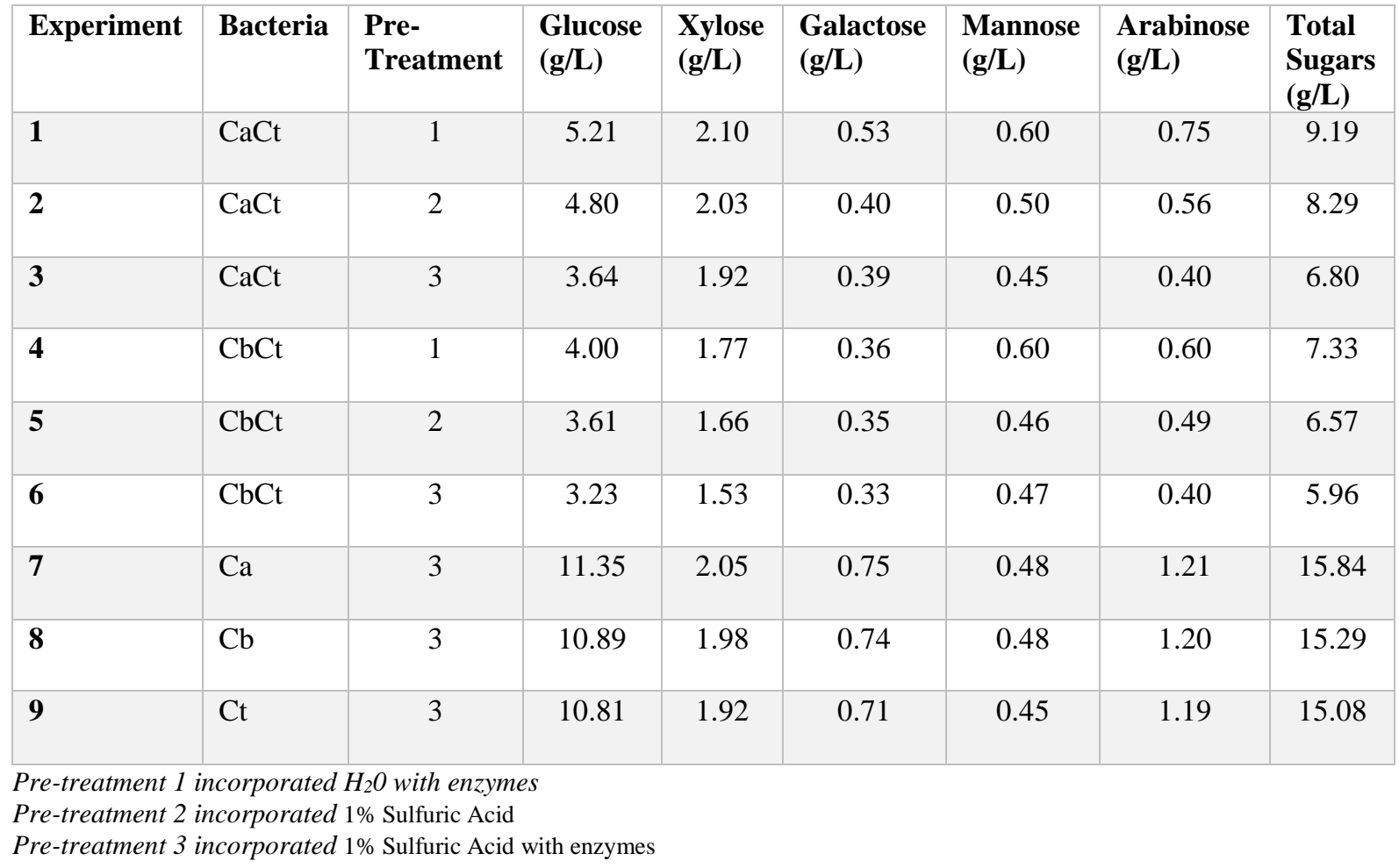

According to Table 4.5, fusant $C b C t$ achieved the highest butanol yield of $7.98 \mathrm{~g} / \mathrm{L}$ when compared to fusant $C a C t$ which attained a value of $7.39 \mathrm{~g} / \mathrm{L}$. Both yields for $\underline{C a C t}$ and $C b C t$ were achieved through biomass treatment \#3 which incorporated thermal, acidic and enzymatic. Furthermore, when examining all fermentation experiments, it can be concluded that the thermal, 
acidic, and enzymatic treatments achieved the highest biobutanol yield when compared with treatment 1 (enzymatic + water) and treatment 2 (acid). When compared with single strains (sample 7, 8, and 9), the fusants $\mathrm{CaCt}$ and $\mathrm{CbCt}$ resulted in higher $\mathrm{ABE}$ yields and consequently biobutanol yield. Therefore, this can clearly indicate the successful application of the protoplast fusion technique and development of a viable fusant specific to biobutanol production.

Moreover, this can also elucidate that the combined properties of the two Clostridia (in the form of a hybrid) have a greater ability to degrade algal biomass and release the necessary sugars for the ABE fermentation pathway. As indicated in Table 4.5, parent strains, $\mathrm{Ca}, \mathrm{Cb}$, and $\mathrm{Ct}$, were only able to have sugar consumptions of $74.15 \%, 75.92 \%$, and $76.59 \%$; respectively. However, combining $\mathrm{Ca}$ with $\mathrm{Ct}$, maximizes the sugar consumption from $74.15 \%$ ( $\mathrm{Ca}$-experiment \#7) to 85.21\% ( $\mathrm{CaCt}$-experiment \#3). Furthermore, combining $\mathrm{Cb}$ with $\mathrm{Ct}$, maximizes the sugar consumption from $75.92 \%$ ( $\mathrm{Cb}$-experiment \#8) to $87.56 \%$ ( $\mathrm{CbCt}$-experiment \#6).

Table 4.5 ABE production values and sugar consumption of algae fermentation after 120 hours

\begin{tabular}{|l|l|l|l|l|l|l|l|l|l|}
\hline Sample & Bacteria & $\begin{array}{l}\text { Pre- } \\
\text { treatment }\end{array}$ & $\begin{array}{l}\text { Acetone } \\
\text { (g/L) }\end{array}$ & $\begin{array}{l}\text { Butanol } \\
\text { (g/L) }\end{array}$ & $\begin{array}{l}\text { Ethanol } \\
\text { (g/L) }\end{array}$ & $\begin{array}{l}\text { Acetic } \\
\text { Acid } \\
\mathbf{( g / L})\end{array}$ & $\begin{array}{l}\text { Butyric } \\
\text { Acid } \\
(\mathbf{g} / \mathbf{L})\end{array}$ & $\begin{array}{l}\text { Total } \\
\text { ABE } \\
(\mathbf{g} / \mathbf{L})\end{array}$ & $\begin{array}{l}\text { Sugar } \\
\text { Consumption } \\
(\mathbf{\%})\end{array}$ \\
\hline $\mathbf{1}$ & $\mathrm{CaCt}$ & 1 & 3.41 & 7.06 & 1.01 & 0.64 & 0.61 & 12.10 & 77.49 \\
\hline $\mathbf{2}$ & $\mathrm{CaCt}$ & 2 & 3.61 & 7.39 & 1.10 & 0.73 & 0.66 & 11.48 & 75.31 \\
\hline $\mathbf{3}$ & $\mathrm{CaCt}$ & 3 & 4.02 & 7.39 & 1.13 & 0.76 & 0.69 & 12.55 & 79.42 \\
\hline $\mathbf{4}$ & $\mathrm{CbCt}$ & 1 & 3.84 & 7.42 & 1.02 & 0.66 & 0.64 & 13.41 & 85.21 \\
\hline $\mathbf{5}$ & $\mathrm{CbCt}$ & 2 & 4.41 & 7.84 & 1.17 & 0.78 & 0.74 & 12.28 & 79.29 \\
\hline $\mathbf{6}$ & $\mathrm{CbCt}$ & 3 & 4.85 & 7.98 & 1.19 & 0.79 & 0.76 & 14.02 & 87.56 \\
\hline $\mathbf{7}$ & $\mathrm{Ca}$ & 3 & 3.18 & 5.94 & 0.83 & 0.59 & 0.42 & 9.95 & 74.15 \\
\hline $\mathbf{8}$ & $\mathrm{Cb}$ & 3 & 3.57 & 5.97 & 0.88 & 0.60 & 0.48 & 10.42 & 75.92 \\
\hline $\mathbf{9}$ & $\mathrm{Ct}$ & 3 & 3.96 & 6.04 & 0.99 & 0.70 & 0.60 & 10.99 & 76.59 \\
\hline
\end{tabular}

Pre-treatment 1 incorporated $\mathrm{H}_{2} \mathrm{O}$ with enzymes Pre-treatment 2 incorporated $1 \%$ Sulfuric Acid Pre-treatment 3 incorporated $1 \%$ Sulfuric Acid with enzymes 
Fermentation results of samples \#7, 8, and 9 represent results for the respective Clostridia parental strains. Biobutanol concentration was in the range from $5.94 \mathrm{~g} / \mathrm{L}(\mathrm{Ca})$ to $6.04 \mathrm{~g} / \mathrm{L}(\mathrm{Ct})$. The differences in biobutanol yield achieved through the parent strains of bacteria is not extensively different. $C t$ achieved a biobutanol concentration of $6.04 \mathrm{~g} / \mathrm{L}$ which was $0.07 \mathrm{~g} / \mathrm{L}$ and $0.10 \mathrm{~g} / \mathrm{L}$ more than the biobutanol concentration values associated with $C b$ and $C t$; respectively. This can indicate that all three Clostridium spp. have a high cellulolytic rate. The cellulolytic rate is a measure of how well an organism can breakdown cellulosic material. However, it is important to note that from the resultant data, it can be concluded that $C t$ has the highest cellulolytic rate as perceived from the biobutanol concentration and the total sugar consumption of $61.21 \%$. This agrees with literature as originally identified by Lynd and Grethlein (1989) and re-evaluated by Levin et al. (2006) and Lynd et al. (2002). It has been concluded that out of all cellulose degrading microorganisms, $C t$ exhibits the highest rate of cellulose degradation (Lynd and Grethlein, 1989; Levin et al., 2006; Lynd et al., 2002). Therefore, utilizing its promising characteristics alongside advantageous qualities of $C a$ and $C b$, the biobutanol production and sugar consumption of the fusants are superior.

$C t$ is a gram-positive, acetogenic, anaerobic, and thermophilic bacteria that degrades cellulose. One of its unique characteristics is that it is a thermophile which indicates that it can be grown and sustained at higher temperatures $\left(50^{\circ} \mathrm{C}-68^{\circ} \mathrm{C}\right)$ when compared with $\mathrm{Ca}$ and $\mathrm{Cb}$ (Akinosh et al., 2014). Fermentation requires higher temperatures of $45^{\circ} \mathrm{C}$ therefore developing a fusant of $\mathrm{CaCt}$ and $\mathrm{CbCt}$ improves the thermostability of the $\mathrm{Ca}$ and $\mathrm{Cb}$ during the entire fermentation process. Although $C t$ possesses superior and unique characteristics such as a higher cellulose degradation rate and thermophilic ability, it does however pose some disadvantages. When compared with $C a$ and $C b, C t$ has a lower butanol tolerance (Pfromm et al., 2010). Thus, 
this places a limit on how much biobutanol can be produced through $C t$ before the butanol can hinder the viability and functionality of the microorganism. As indicated in the present study, protoplast fusion was able to facilitate the development of a strain that possessed 1) higher temperature resistant 2) higher butanol tolerance and 3) higher cellulolytic rate.

To determine the effectiveness of protoplast fusion for the development of $\mathrm{CaCt}$ and $C b C t$, it is important to compare the resultant data for values obtained from literature. Table 4.6 shows the resultant biobutanol concentration from algal biomass from the Clostridia strains as described in various studies. The biobutanol concentration $(7.98 \mathrm{~g} / \mathrm{L})$ obtained from the fusant $C b C t$ was the highest when compared with the fusant $C a C t(7.39 \mathrm{~g} / \mathrm{L})$ Additionally, it is also important to note that the biobutanol concentration from $C b C t$ is also $0.46 \mathrm{~g} / \mathrm{L}$ more than what was originally obtained in previous lab studies conducted in the Nanocomposites and Biomaterials Lab at Ryerson University. This can indicate greater efficiency, optimization and use of resources to improve experimental results.

The present study resulted in a final biobutanol concentration of $5.94 \mathrm{~g} / \mathrm{L}$ through $\mathrm{Ca}$. As indicated in Table 4.6, this is higher than the value $(3.86 \mathrm{~g} / \mathrm{L})$ obtained for the same Clostridia strain for algal biomass (Cheng et al., 2017). Furthermore, the value of the final biobutanol concentration obtained through $C b$ in this study was $5.97 \mathrm{~g} / \mathrm{L}$ which is also greater than what has been reported in literature as show in the table below. Potts et al. (2011) resulted in a value of 4 $\mathrm{g} / \mathrm{L}$ for biobutanol concentration. A variety of factors can influence the final biobutanol concentration which can explain the variation in biobutanol concentrations achieved in this study. Although the studies cited in the table below all reference algal biomass (microalgae or macroalgae) as the substrate, each can have varying concentrations of cellulose and hemicellulose. Therefore, the resultant carbohydrate concentration can differ as well therefore 
impacting the final biobutanol concentration. Additionally, other influential factors can include the process, which was applied to yield the final biobutanol, the specific hydrolysis treatment of the algal biomass to breakdown the polysaccharide structure and the specificity of the Clostridia strain.

Potts et al. (2011) achieved a final biobutanol concentration of $4 \mathrm{~g} / \mathrm{L}$ when using $C b$ as the Clostridia spp. during the fermentation process. This concentration was $1.97 \mathrm{~g} / \mathrm{L}$ less than the biobutanol concentration achieved in the present study using the same Clostridia spp. Upon further analysis, it can be concluded there are a variety of different elements that can explain the variation in biobutanol concentration from both studies. As mentioned previously, the internal composition can greatly affect the resultant value of the polysaccharides that can be readily converted to monomeric sugars utilized in the ABE pathway. Potts et al. (2011) utilized Ulva Lactuca which is a species of macroalgae, found in marine waters. On the contrary, the present study utilized microalgae, Stichococcus spp., as the main substrate for biobutanol production. Each of the two strains vary greatly in terms of their internal carbohydrate structure and content (Burlew, 1976 and Potts et al., 2011). Given that the carbohydrate content of U. Lactuca is generally much higher (within the range of 50-60\%) it is recognized as a potentially suitable candidate for alcoholic biofuels (Potts et al., 2011). As described in this study, the algal biomass used had a lower carbohydrate content of 38.9\% (Burlew, 1976). However, the biobutanol concentration achieved was $1.97 \mathrm{~g} / \mathrm{L}$ more than that of Potts et al. (2011). This can be explained by the specificity of the Clostridia spp. used in both studies and the hydrolysis treatments. The present study used C. beijerinickii BA101 whereas Potts et al. (2011) used C. beijerinickii 35702. C. beijernickii BA101 is a mutant strain developed from the wild strain of C. beijernickii 8052 and has enhanced capabilities to break down starch and has higher butanol tolerance between 
0.017-0.021 kg butanol/L of fermentation broth (Ezeji et al., 2004). Furthermore, hydrolysis treatments used in Potts et al. (2011) were different than that of the present study. Biobutanol concentration of $5.97 \mathrm{~g} / \mathrm{L}$ obtained in this study had a hydrolysis treatment that consisted of $1 \%$ $\mathrm{H}_{2} \mathrm{SO}_{4}$ and enzymes (cellulase $+\beta$-glucosidase) applied with a thermal treatment of $120^{\circ} \mathrm{C}$ for 20 minutes. On the contrary, the hydrolysis treatment applied by Potts et al. (2011) was only subjected to an acidic $\left(1 \% \mathrm{H}_{2} \mathrm{SO}_{4}\right)$ and thermal treatment $\left(125^{\circ} \mathrm{C}\right.$ for 30 minutes) without the addition of enzymes. Therefore, it can be concluded that the mutant Clostridium sp. C. beijernickii BA101 and addition of enzymes played a significant role in yielding a greater amount of biobutanol. C. beijernickii BA101 has the superior capability to further breakdown the carbohydrate structure and utilize it in a more efficient manner when compared to C. beijernickii 35702. Furthermore, the addition of enzymes ensured a higher saccharification efficiency during the fermentation processes.

Cheng et al. (2017) achieved a final biobutanol concentration of $3.86 \mathrm{~g} / \mathrm{L}$ when using $\mathrm{Ca}$ as the prime Clostridium sp. The value of biobutanol obtained through $\mathrm{Ca}$ in the present study was $5.94 \mathrm{~g} / \mathrm{L}$ which happens to be $2.08 \mathrm{~g} / \mathrm{L}$ greater than what has been cited in literature. Similar to the variations with $C b$ obtained biobutanol with literature, the variations in biobutanol obtained through $\mathrm{Ca}$ in this study can be explained by analyzing the experimental differences. Cheng et al. (2017) utilized Chlorella sorokiniana whereas the present study utilized a Stichococcus sp. of microalgae. It was clearly explained that the resultant carbohydrate content (56.47\% versus $38.9 \%$ obtained from Stichococcus ) was the highest with a hydrolysis treatment of $2 \% \mathrm{H}_{2} \mathrm{SO}_{4}$ followed by $2 \% \mathrm{NaOH}$ (Cheng et al., 2017). However, it was explained that $47.6 \%$ of the carbohydrate was insoluble. Furthermore, $\mathrm{Ca}$ was unable to utilized one-third of the carbohydrate content thus hindering the ability to produce a greater biobutanol concentration. 
Consequently, this can explain the variation in the resultant biobutanol concentration with literature. Furthermore, this also indicates the importance of enzymatic hydrolysis for algal biomass.

Table 4.6 Comparison of biobutanol concentration produced from algal biomass

\begin{tabular}{|l|l|c|l|}
\hline Bacteria & Biomass & $\begin{array}{l}\text { Biobutanol Concentration } \\
(\mathbf{g} / \mathbf{L})\end{array}$ & Reference \\
\hline $\mathbf{C b}$ & Macroalgae & 4 & Potts et al., 2011 \\
\hline $\mathbf{C a}$ & Microalgae & 3.86 & Cheng et al., 2017 \\
\hline $\mathbf{C b C t}$ & Microalgae & 7.52 & Roy, 2014* \\
\hline $\mathbf{C a}$ & Microalgae & 5.94 & Present Study \\
\hline $\mathbf{C b}$ & Microalgae & 5.97 & Present Study \\
\hline $\mathbf{C t}$ & Microalgae & 6.04 & Present Study \\
\hline $\mathbf{C a C t}$ & Microalgae & 7.39 & Present Study \\
\hline $\mathbf{C b C t}$ & Microalgae & 7.98 & Present Study \\
\hline *Previous study conducted in Nanocomposites and Biomaterials Lab, Ryerson University
\end{tabular}

Table 4.7 shows a comparison of biobutanol concentrations produced from different agricultural resources cited in literature. Previous studies conducted in the Nanocomposites and Biomaterials Laboratory; Ryerson University have also been cited. From the table below, it can be seen that when using $C a$, microalgae (used in the present study) was most successful in obtaining a biobutanol yield in contrast with rice straw and seepweed. However, when comparing the two strains of Clostridium spp. $C a$ and $C b, C b$ was more successful in breaking down wheat straw to yield a higher biobutanol concentration of 7.6 g/L (Lynd et al., 2005). When examining the effectiveness of $C b$ on biobutanol yield, it can be seen that wheat straw faired better as it resulted in a higher biobutanol concentration of $7.4 \mathrm{~g} / \mathrm{L}$ when compared to the algae $(5.97 \mathrm{~g} / \mathrm{L})$. This can be explained by the higher cellulose and hemicellulose contents of 
wheat straw (Table 4.2). Additionally, it can be assessed that the lower biobutanol yield from algae as compared with wheat straw can be explained by presence of inhibitors (Ezeji et al., 2007). $C b$ was also able to result in a higher biobutanol yield $(6.45 \mathrm{~g} / \mathrm{L})$ with bamboo as oppose to the microalgae used in the present study $(5.97 \mathrm{~g} / \mathrm{L})$. The higher cellulose and hemicellulose contents found in bamboo can account for this variation (Kumar et al., 2017).

Biobutanol production obtained from the microalgae used in the present study with $\mathrm{Ca}$ achieved significantly better results when compared rice straw and seepweed. As indicated in Table 4.7, the biobutanol concentration is $3.84 \mathrm{~g} / \mathrm{L}$ and $2.44 \mathrm{~g} / \mathrm{L}$ higher than that of rice straw and seepweed, respectively. Rice straw has similar carbohydrate content than that of the microalgae used in this study (within the range of 35-40\%) (Zhao et al., 2011; Santiago et al., 2016). However, the resultant biobutanol concentration can be influenced by the effectiveness of hydrolysis treatments. Zhao et al. (2011) used a chemical treatment that consisted of $4 \% \mathrm{NaOH}$ whereas the present study used $1 \% \mathrm{H}_{2} \mathrm{SO}_{4}$. Furthermore, the enzymatic treatment utilized only cellulase however the present study used a combination of cellulase and $\beta$-glucosidase. The application of $\mathrm{H}_{2} \mathrm{SO}_{4}$ with cellulase and $\beta$-glucosidase resulted in a higher biobutanol yield thus indicating the superior capability of this particular hydrolysis treatment to breakdown the internal lignocellulosic material. As stated previously, $\mathrm{H}_{2} \mathrm{SO}_{4}$ in concentration of $1 \%$ is best suited for cellulose and hemicellulose disintegration (Kumar et al., 2009). Furthermore, the additional use of the second enzyme $\beta$-glucosidase is beneficial because it further targets the cellulose structure thus permitting the release of more sugars. In addition to the hydrolysis treatments, the lignocellulosic components and physical attributes of algae and rice straw can influence the resultant biobutanol concentration. The absence of lignin in the algae used in the present study allows for a relatively easier disintegration path. On the contrary, rice straw has a lignin content 
within the range of 16-20\% (Zhao et al., 2011). Consequently, this makes the physical structure of the rice straw far more rigid than the algae. With the higher amount of lignin present, it is more difficult to breakdown the internal structure of the rice straw, release sugars and subsequently produce biobutanol.

The resultant lower biobutanol concentration from seepweed with the utilization of $\mathrm{Ca}$ during the SHF procedure can be influenced by the relative lower carbohydrate content of seepweed, 25\% (Qureshi et al., 2008 and Zhao et al., 2011). Upon examination of the biobutanol concentration obtained from $C t$, it can be concluded that $C t$ was slightly more successful in obtaining a higher concentration from the microalgae than the crystalline cellulose. A biobutanol concentration of $5.4 \mathrm{~g} / \mathrm{L}$ was achieved from crystalline cellulose (Lin et al., 2015). This is 0.64 $\mathrm{g} / \mathrm{L}$ less than what was achieved in the present study with algal biomass. This small variation in biobutanol production can be a result of the difference in the chosen biomass processes. The current study utilizes SHF whereas Lin et al (2015) has chosen consolidated biomass processing (CBP). $\mathrm{CBP}$ has been a commonly used biomass processing technique for many years now. $\mathrm{CBP}$ combines the processes of cellulase production, cellulase hydrolysis, hexose formation and pentose formation (Olsen et al., 2012). Furthermore, it has been recognized as the optimal configuration for low-cost hydrolysis and fermentation of cellulosic biomass (Olsen et al., 2012). However, as recent research can be indicative of, one of the prime drawbacks of this processing technique is that fermenting microorganisms and cellulases required different operating temperatures (Lynd et al., 2005). SHF accounts for these temperature changes as the biological compounds are produced at different stages (Ohgren et al., 2006)

Table 4.7 also shows the comparison of biobutanol concentration produced from protoplast fusion and co-culture. A co-culture of cells is simply a technique in which two of 
more population of cells from different species are grown in close proximity. This varies greatly from protoplast fusion technology, as described in this study. Previous studies in the Nanocomposites and Biomaterials Laboratory, Ryerson University examined the corresponding biobutanol concentrations of fused and co-cultured strains. With wheat straw as the substrate, the fused strains $C b C t$ showed a greater result in biobutanol production when compared with the coculture of $\mathrm{CbCt}$. As indicated below, a co-culture $\mathrm{CbCt}$ resulted in a biobutanol concentration of $5.79 \mathrm{~g} / \mathrm{L}$ whereas the fusant $C b C t$ achieved a significantly higher concentration of $13.83 \mathrm{~g} / \mathrm{L}$. This further shows the successful application of protoplast fusion technology as a viable genetic engineering tool in biofuel production. 
Table 4.7 Comparison of biobutanol concentration produced from different agricultural sources

\begin{tabular}{|c|c|c|c|}
\hline Bacteria & Biomass & $\begin{array}{l}\text { Biobutanol } \\
\text { Concentration }(\mathrm{g} / \mathrm{L})\end{array}$ & Reference \\
\hline $\mathbf{C a}$ & Rice Straw & 2.1 & Zhao et al., 2011 \\
\hline $\mathbf{C a}$ & Seepweed & 3.5 & $\begin{array}{l}\text { Qureshi et al., } \\
2008\end{array}$ \\
\hline $\mathbf{C a}$ & Wheat Straw & 5.05 & Wang et al. 2013 \\
\hline $\mathbf{C b}$ & Wheat Straw & 7.4 & Lynd et al., 2005 \\
\hline $\mathbf{C b}$ & Bamboo & 6.45 & $\begin{array}{l}\text { Kumar et al., } \\
2017\end{array}$ \\
\hline $\mathbf{C t}$ & $\begin{array}{l}\text { Crystalline } \\
\text { Cellulose }\end{array}$ & 5.4 & Lin et al., 2015 \\
\hline $\begin{array}{l}\text { Co-Culture Ct and } \\
\text { Cs }\end{array}$ & Rice Straw & 5.5 & $\begin{array}{l}\text { Kiyoshi et al., } \\
2015\end{array}$ \\
\hline $\mathbf{C b C t}$ & Wheat Straw & 13.83 & Roy, 2014* \\
\hline $\begin{array}{l}\text { Co-culture } \mathrm{Cb} \text { and } \\
\mathrm{Ct}\end{array}$ & Wheat Straw & 5.79 & Mohtasebi, 2013* \\
\hline $\mathbf{C a}$ & Microalgae & 5.94 & Present Study \\
\hline $\mathbf{C b}$ & Microalgae & 5.97 & Present Study \\
\hline $\mathbf{C t}$ & Microalgae & 6.04 & Present Study \\
\hline $\mathrm{CaCt}$ & Microalgae & 7.39 & Present Study \\
\hline $\mathrm{CbCt}$ & Microalgae & 7.98 & Present Study \\
\hline
\end{tabular}

*Previous study conducted in Nanocomposites and Biomaterials Lab, Ryerson University

Table 4.8 shows a comparison of the resultant ABE and butanol yield from the present study using algal biomass with wheat straw from a previous study conducted in the Nanocomposites and Biomaterials Lab. When compared with wheat straw, the algal biomass has resulted in a lower $\mathrm{ABE}$ and butanol yield. This can be accounted for by the total sugars present in both feedstocks. As indicated previously, wheat straw has a higher sugar composition when 
compared with algae. In addition to this, the lower yields can also be explained by the presence of inhibitors in algae (Ezeji et al., 2007; Potts et al., 2011). The presence of inhibitors can limit growth and can also reduce the amount of available sugars necessary for ABE production (Potts et al., 2011). Although the algal biomass used in the present study has resulted in a lower butanol yield when compared to wheat straw, the productivity of algae is significantly greater than wheat straw. Algae grow at significantly higher rates and are not subjected to seasonal production (Gouveia, 2011; Medipally et al., 2015). Furthermore, as discussed previously, algae are also comprised of a lipid component which can also be extracted to produce biofuels such as biodiesel. Consequently, the energy availability of algae is higher than other biomass including wheat straw.

Table 4.8 ABE and butanol yield comparison of wheat straw and algae following fermentation

\begin{tabular}{|l|l|l|l|}
\hline Feedstock & $\begin{array}{l}\text { Butanol Yield } \\
(\mathbf{g} / \mathbf{g})\end{array}$ & $\begin{array}{l}\text { ABE Yield } \\
(\mathbf{g} / \mathbf{g})\end{array}$ & Reference \\
\hline Wheat straw & 0.36 & 0.60 & Roy, 2014* \\
\hline Algae & 0.30 & 0.52 & Present Study \\
\hline
\end{tabular}

*Previous study conducted in Nanocomposites and Biomaterials Lab, Ryerson University

\subsection{Bio-Oil Production}

\subsubsection{Ultrasonication Results}

Figure 4.4 shows the resultant oil extraction and oil yield of different ultrasonication experiments. Two different terpenes (d-limonene and p-cymene) were utilized for the ultrasonication experiments. Each experiment was carried in duplicates to ensure accuracy and reproducibility. Raw data for each experiment is shown in Appendix E. According to Figure 4.5, the optimal time for maximal yield was 30 minutes for both terpenes. The oil yield obtained with d-limonene (experiment \#3) was $38.56 \%$ whereas as the oil yield obtained with p-cymene (experiment \#7) was $34.70 \%$. However, ultrasonication after the 40-minute exposure time 
negatively effected the oil yield. Oil yields for both terpenes declined by $9.64 \%$ and $8.99 \%$ for dlimonene and p-cymene, respectively. As indicated by Kumar et al. (2017), prolonged ultrasonication time can in fact impede oil yield and quality through the formation of radicals. Experiment \#9 was the control and utilized water as the solvent. The oil yield from experiment \#9 was significantly lower than the usage of terpenes. Exposure time of 30 minutes with dlimonene and p-cymene resulted in oil yields that were $37.28 \%$ and $33.42 \%$ higher than the control; respectively.

The higher oil yield of d-limonene following the 30-minute exposure time can indicate the greater suitability of d-limonene, when compared with p-cymene, as a terpene to use in conjunction with ultrasonication. The utilization of chemicals alongside ultrasonication has been known to improve cell disruption efficiency and subsequent oil recovery (Mubarak et al., 2015; Harris et al., 2018; Skorupskaite et al., 2019 Sati et al., 2019). Different paraments can influence the oil recovery through ultrasonication, including energy and frequency. Generally, a higher power specification can result in a higher oil recovery rate however that requires more energy. Consequently, the utilization of organic solvents reduces the amount of energy required for the process (Halim et al., 2012; Skoropskaite et al., 2019; Sati et al., 2019). Furthermore, the physical properties of the chemical can also influence the effect of ultrasonication upon cell disruption and subsequent lipid recovery. Physical properties such as surface tension and viscosity play a critical role in the severity of cavitation development and collapse (Bussemaker and Zhang, 2013). During the ultrasonication process, hydrophobic solvents, such as d-limonene and p-cymene, gather at the bubble interface and can be either radical scavengers in a hot region surrounding the bubble or reduce the maximum temperature reached during the bubble's collapse. The squeezing and subsequent collapse of the bubble occur as a result of the 
evaporation of the solute. During the exposure time, oscillations in the bubble allow for solvent evaporation and consequently degradation reactions of the cell wall (Bussemaker and Zhang, 2013). It has been examined that the more volatile a solvent, the more it is able to squeeze the bubble through the ability of increased evaporation (Ashokkumar et al., 1997 and Ashokkumer et al., 2000). D-limonene and p-cymene have fairly comparable physical properties as shown by Tanzi et al. (2012) however, it has been indicated that in terms of volatility as per the number of volatile compounds, d-limonene is slightly more volatile than p-cymene (Ciriminna et al., 2014 and El-Zaeddi et al., 2016). Therefore, the slightly more volatile state of d-limonene can account for the $3.86 \%$ increase in oil yield. In addition to the volatility of the solvent, surface tension can also influence the ultrasonication effects, as previously mentioned (Bussemaker and Zhang, 2013). It has been discussed that lower surface tension can result in enhanced ultrasonication effects (Cheng et al., 2010 and Bhardwaj et al., 2015). The surface tension of d-limonene at $25^{\circ} \mathrm{C}$ is 25.8 dyne $\cdot \mathrm{cm}^{-1}$ which is lower than that of p-cymene (28.5 dyne $\left.\cdot \mathrm{cm}^{-1}\right)$ (Tanzi et al., 2012).

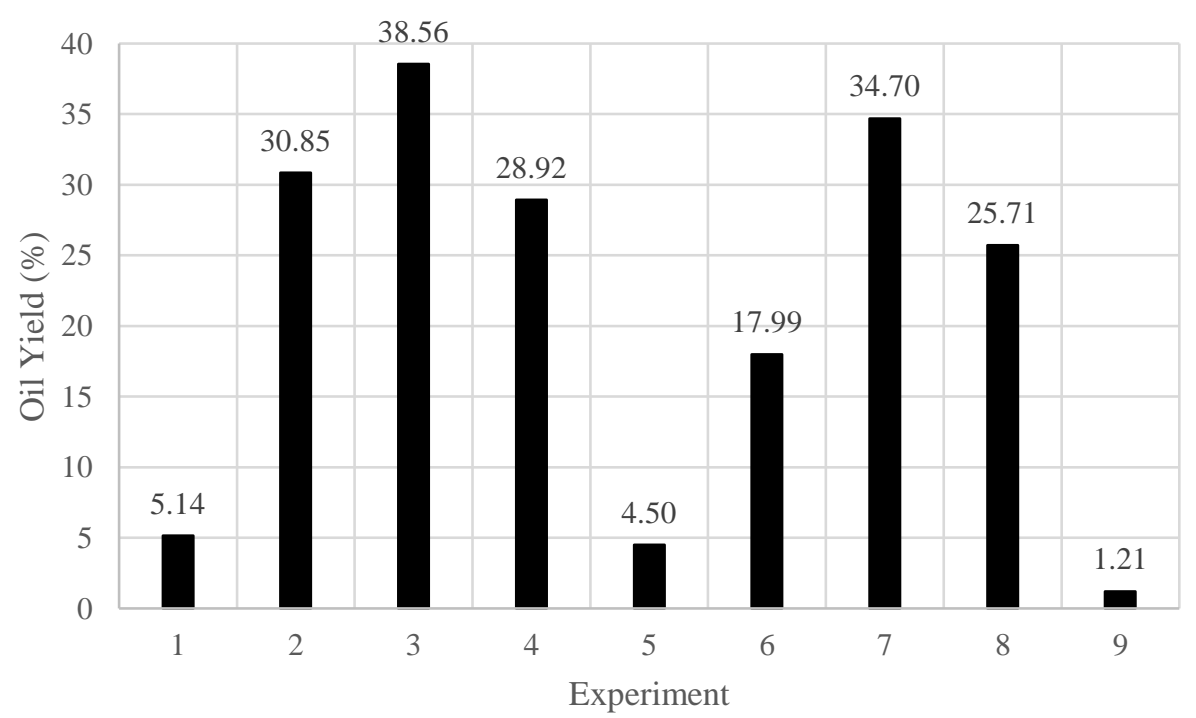

Figure 4.4 Oil Yield from ultrasonication experiments 
Table 4.9 shows the relative oil yield derived from different algae species utilizing ultrasonication as the prime cell disruption method. A variation in oil yield can be illustrated in the table below. This can be accounted by the variation in frequency, power, exposure time, as well as usage of solvents and their respective ratios. The present study obtained an oil yield of $38.56 \%$ (d-limonene) and 34.70\% (p-cymene) with sonication parameters of $20 \mathrm{kHz}, 200 \mathrm{~W}$ and an exposure time of 30 minutes. Similar sonication parameters, for Chlorella sp., resulted in a significantly higher oil yield of $67.4 \%$ (Roux et al., 2017). As previously discussed, solvent choice can greatly influence the effectiveness of ultrasonication and the oil recovery process. When compared with the terpenes used in the present study, chloroform and methanol have been recognized to be superior solvents. A solvent system containing chloroform and methanol, typically in a 1:2 ratio, is the most widely utilized organic solvent mixture used for lipid extraction for any biological compound (Halim et al., 2012). The significant increase in oil yield obtained from using this solvent mixture can be attributed to the physical properties of chloroform and methanol. The specificities of the mixture allow for the advantageous usage of residual endogenous water in the microalgal cells to act as a ternary component (Halim et al., 2012). This can be beneficial because this allows the complete extraction of both neutral and polar lipids (Medina et al., 1998 and Halim et al., 2012). Although the efficiency of this solvent has been considered quite attractive and a prime choice for lipid extraction, chloroform is highly toxic and not environmentally friendly. The present study is the first of its kinds to utilize terpenes as the main solvent during ultrasonication. Despite the variation in oil yield, the utilization of d-limonene and p-cymene as a solvent in ultrasonication shows promise. When compared with a study by Naghdi et al. (2016), the terpene solvent assisted ultrasonication used in the present study achieved a greater oil yield. The oil yield from the present study was $10.33 \%$ 
(d-limonene) and 6.37\% (p-cymene) greater. Although the parameters for the study by Nagdhi et al. (2016) varied in terms of power and exposure time it can be concluded that the terpenes faired better than a chloroform-ethanol solvent mixture. Despite the higher power, low exposure time as described in the study Naghdi et al (2016), the present study, which utilized a lower power $(200 \mathrm{~W})$ and higher exposure time (30 minutes), resulted in a higher oil yield.

Table 4.9 Comparison of oil yield from different algae species

\begin{tabular}{|l|l|l|c|l|}
\hline Biomass & $\begin{array}{l}\text { Sonication } \\
\text { Parameters }\end{array}$ & $\begin{array}{l}\text { Solvents } \\
\text { (Ratio) }\end{array}$ & Oil Yield (\%) & Reference \\
\hline $\begin{array}{l}\text { Scenedesmus } \\
\text { sp. }\end{array}$ & $\begin{array}{l}1000 \mathrm{~W} ; 10 \\
\text { minutes }\end{array}$ & $\begin{array}{l}\text { Chloroform and } \\
\text { ethanol }(1: 1)\end{array}$ & 28.33 & $\begin{array}{l}\text { Naghdi et al., } \\
2016\end{array}$ \\
\hline Chlorella sp. & $\begin{array}{l}20 \mathrm{kHz} ; 30 \\
\text { minutes }\end{array}$ & $\begin{array}{l}\text { Chloroform } \\
\text { methanol }(1: 2)\end{array}$ & 67.40 & $\begin{array}{l}\text { Roux et al., } \\
2017\end{array}$ \\
\hline Chlorella sp. & $\begin{array}{l}20 \mathrm{kHz} ; 30 \\
\text { minutes }\end{array}$ & $\begin{array}{l}\text { Hexane; } \\
\text { isopropanol } \\
(3: 2)\end{array}$ & 76.60 & $\begin{array}{l}\text { Roux et al., } \\
2017\end{array}$ \\
\hline Chlorella sp. & $\begin{array}{l}700 \mathrm{~W} ; 50 \\
\text { seconds }\end{array}$ & $\begin{array}{l}\text { Chloroform, } \\
\text { methanol, water } \\
(2: 2: 1.9)\end{array}$ & 31.50 & Lee et al., 2016 \\
\hline Chlorella sp. & $\begin{array}{l}20 \mathrm{kHz} ; 5 \\
\text { minutes }\end{array}$ & $\begin{array}{l}\text { Chloroform and } \\
\text { methanol }(1: 1)\end{array}$ & 2.40 & $\begin{array}{l}\text { Drira et al., } \\
2016\end{array}$ \\
\hline Chlorella sp. & $\begin{array}{l}20 \mathrm{kHz} ; 20 \\
\text { minutes }\end{array}$ & $\begin{array}{l}\text { Chloroform and } \\
\text { methanol }(1: 1)\end{array}$ & 2.20 & $\begin{array}{l}\text { Drira et al., } \\
2016\end{array}$ \\
\hline Sticoccocus sp. & $\begin{array}{l}20 \mathrm{kHz} ; 30 \\
\text { minutes }\end{array}$ & d-limonene & 38.56 & Present Study \\
\hline Sticoccocus sp. & $\begin{array}{l}20 \mathrm{kHz} ; 30 \\
\text { minutes }\end{array}$ & p-cymene & 34.70 & Present study \\
\hline
\end{tabular}

\subsubsection{Modified Bligh and Dyer Results}

Table 4.10 shows the resultant oil extracted and oil yield for the modified Bligh and Dyer approach utilized in this study. Each experiment was carried out in duplicate to ensure accuracy and reproducibility. Raw data for each experiment can found in Appendix E. From the figure below, it can be seen that experiment \#1 involving d-limonene resulted in a higher oil yield of $26.99 \%$. The utilization of p-cymene in the modified method resulted in an oil yield slightly lower at $25.06 \%$. The effectiveness of utilizing a solvent for lipid extraction is dependent upon 
its volatility and chemical affinity towards the polar lipid (Halim et al., 2012 and Harris et al., 2018). As previously discussed, of the two terpenes, d-limonene is slightly more volatile than pcymene thereby accounting for the $1.93 \%$ oil yield increase.

When a microalgae cell is exposed to a non-polar solvent such as d-limonene or pcymene, the goal of the solvent is to penetrate through the cell membrane into the cytoplasm and interact with the neutral lipids using Van der Waals forces (Halim et al., 2012). Following this, a solvent-lipid complex is created which is subjected to a concentration gradient thus leading to the diffusion of the solvent-lipid complex outside of the cell membrane (Halim et al., 2012). The effectiveness of the penetration, strength of the Van der Waal forces and subsequent production of the solvent-lipid complex is subject to the chemical affinity of the solvent, volatility, and most importantly the Hansen Solubility Parameter (HSP) (Kates, 1986; Medina et al., 1998; Halim et al., 2012; Harris et al., 2018). Further examination of the $1.93 \%$ increase in oil yield accounted by d-limonene can be attributed to its higher chemical affinity to the neutral lipids and consequently its lower HSP value (Tanzi et al., 2012).

Table 4.10 Oil yield from Modified Bligh and Dyer experiments with algal biomass using terpenes

\begin{tabular}{|l|l|}
\hline Experiment & Oil Yield $(\%)$ \\
\hline 1. d-Limonene & 26.99 \\
\hline 2. p-Cymene & 25.06 \\
\hline
\end{tabular}

Table 4.11 shows the resultant oil yield from different solvent extractions for different microalgae species. As in agreement with what has been previously discussed, a chloroform and methanol solvent mixture is the most successful solvent system that can be applied to biomass to extract lipids. Choi et al. (2014) obtained an oil yield of $70.20 \%$ from C. vulgaris using chloroform and methanol. When comparing the oil yield obtained from terpenes, it can be concluded that the utilization of a single terpene solvent system is more effective than a hexane- 
methanol solvent system and a hexane-methanol-water solvent system. This can further indicate that direct solvent extraction using d-limonene and p-cymene is a promising alternative to harmful and volatile chemicals such as hexane and methanol.

The efficiency of solvent extraction method heavily relies upon the solvent of choice and the ratios that are employed (Sati et al., 2019). As previously discussed, the volatility and chemical affinity to lipids are influential factors in the lipid recovery. Overall, chemicals such as chloroform, methanol, and hexane perform better in cell disruption and possess greater affinities towards lipids. However, as indicated in the table below solvent ratios play an integral role in oil yield. Hexane alone resulted in higher yield for C. vulgaris as oppose to combining hexane and methanol. Improving the extraction rate of solvents require longer time periods (Sati et al., 2019). Furthermore, it has been regarded that a modified approach called the accelerated solvent extraction (ASE) method which requires higher temperature $\left(50-200^{\circ} \mathrm{C}\right)$ and pressure $(500-300$ psi) with shorter time periods (5-10 minutes) can improve extraction efficiencies (Cooney et al., 2009 and Cheng et al., 2011). Although this method has achieved relatively high efficiencies, a major drawback is the utilization of toxic chemicals.

As this study can indicate, the use of terpenes is a promising technique. Despite the less volatile nature of d-limonene and p-cymene, utilizing terpenes as bio-based solvents is a viable alternative. Specific optimization of the experimental parameters using terpenes can result in improved oil recovery. The ASE method using chemicals such as chloroform, methanol, and hexane can cause significant safety issues however less volatile, and eco-friendly substitutes such as d-limonene and p-cymene are more viable. As described by Tanzi et al. (2012) terpenes are significantly safer and naturally occurring. Furthermore, due to their similar molecular weight and structures, hexane and terpenes behave similarly (Tanzi et al., 2012). Terpenes are 
slightly more polar and possess a greater dissociating power compared to hexane (Tanzi et al., 2012). Therefore, further research utilizing bio-based, terpenes are necessary.

Table 4.11 Comparison of different solvents used for oil recovery for algal biomass

\begin{tabular}{|c|c|c|c|}
\hline Biomass & Solvent (Ratio) & Oil Yield (\%) & Reference \\
\hline C. vulgaris & $\begin{array}{l}\text { Chloroform and } \\
\text { methanol }(2: 1)\end{array}$ & 70.20 & Choi et al., 2014 \\
\hline C. vulgaris & Hexane & 60.20 & Choi et al., 2014 \\
\hline C. vulgaris & $\begin{array}{l}\text { Hexane and } \\
\text { methanol }(7: 3)\end{array}$ & 28.95 & $\begin{array}{l}\text { Ryckebosch, et al., } \\
2012\end{array}$ \\
\hline Nanochlorpsis & $\begin{array}{l}\text { Hexane, methanol, } \\
\text { and water }(2: 3: 1)\end{array}$ & 10.46 & Khannal et al., 2007 \\
\hline Sticoccocus sp. & d-limonene & 38.56 & Present Study \\
\hline Sticoccocus sp. & p-cymene & 34.70 & Present Study \\
\hline
\end{tabular}

\subsubsection{Combination Method: Ultrasonication + Modified Bligh and Dyer Results}

Table 4.12 shows the resultant oil yield from the combined two-step process of ultrasonication assisted modified Bligh and Dyer. Each experiment was carried in duplicate to ensure accuracy and reproducibility. Raw data of the experiments can be found in Appendix E. The ultrasonication process utilizing d-limonene resulted in a higher oil yield when compared to p-cymene. As previously discussed, d-limonene presents superiority when compared to pcymene in terms of volatility, chemical affinity and HSP value. Even combined with an ultrasonication process, d-limonene was able to extract a higher amount of oil when compared to p-cymene. The solvent was able to result in a $6.42 \%$ increase. 
Table 4.12 Oil yield from combined two-step process of ultrasonication followed by Modified Bligh and Dyer with algal biomass using terpenes

\begin{tabular}{|l|l|}
\hline Experiment & Oil Yield (\%) \\
\hline 1. d-Limonene & 46.27 \\
\hline 2. p-Cymene & 39.85 \\
\hline
\end{tabular}

Combining different extraction methods can result in a great efficiency of oil recovery (Halim et al., 2012). The effectiveness of a single extraction method is subject to a variety of factors including the experimental specificities and the biomass. As shown in this study, the combination of ultrasonication and a modified Bligh and Dyer method increased the oil yield for the respective microalgae. An effective extraction protocol should increase the cell disruption efficiency. Combination extraction have proven to an effective option for certain algal species that more rigid cell walls and that require more energy for cell disruption. Araujo et al. (2012) evaluated the effects of five different extraction methods coupled with the Bligh and Dyer method. It was concluded that the ultrasound assisted Bligh and Dyer method applied to C. vulgaris resulted in the highest oil extraction of 52\% (Araujo et al., 2012). The present study obtained oil extraction of $46.27 \%$ with d-limonene. This is only $5.73 \%$ lower than that of what has been reported in literature thus indicating the viability of using terpenes in conjunction with ultrasonication.

\subsubsection{Comparison}

Upon examination, it can be concluded that the most successful oil extraction method from the present study was the ultrasound assisted modified Bligh and Dyer method in use with d-limonene. The combined mechanical and bio-based solvent extraction method resulted in a final oil yield of $46.27 \%$. This can indicate that the combination of the two methods further facilitated improved cell disruption as the oil yield increased by $7.71 \%$ and $19.28 \%$, respectively 
from ultrasonication and the modified Bligh and Dyer single extractions. In terms of p-cymene, the oil yield of the combined extraction resulted in $39.85 \%$ which was an increase of $4.25 \%$ and 14.79\%. In both instances, it can be clearly seen that the addition of ultrasonication greatly impacted the oil yield. The oil yield of a single modified Bligh and Dyer using terpenes resulted in relatively lower oil yields. Although the conventional Bligh and Dyer method, utilizing chloroform and methanol, is the most successful in oil extraction from biological tissues, the replacement of terpenes is not nearly as successful. As indicated in this study, the variations in volatility, chemical affinities, and HSP values of the terpenes and traditionally used chemicals (chloroform and methanol) can greatly impact the oil yield. Therefore, it is critical that additional extraction protocols be undertaken to improve extraction yields. The present study explored the potential of combination extraction methods which greatly improved oil yield. Further research can be geared towards applying variations to the modified Bligh and Dyer method to reflect aspects of the ASE method (Cooney et al., 2009 and Cheng et al., 2011). The combined ultrasound assisted modified Bligh and Dyer extraction method resulted in the highest oil yield as well as the highest cell disruption efficiency (Figure 4.5). As the figure below illustrates, the cell disruption efficiency was calculated for each oil extraction as described in the methods section (3.3.6). The raw data for the cell disruption is shown in Table 4.10. The cell disruption efficiency is an indicator of how successful an application is in rupturing the cells which ultimately provide access to the lipid content of the microalgae cell. Consequently, the combined method resulted in the highest cell disruption efficiency of $77.78 \%$. The point of reference for this disruption efficiency is the control experiment which included algae in water prior to any extraction applications. Furthermore, the modified Bligh and Dyer method (of both terpenes) was more successful than the ultrasound experiments of exposure time of 10 minutes. The direct use 
of d-limonene and p-cymene resulted in a cell disruption efficiency of $33.33 \%$ and $28.40 \%$, respectively. These values are $9.87 \%$ and $6.18 \%$ greater than the ultrasonication experiments of 10 minutes with d-limonene and p-cymene, respectively.

Table 4.13 Cell disruption efficiency of different algae oil extraction methods

\begin{tabular}{|l|c|c|}
\hline Experiment & $\begin{array}{l}\text { Cell Count } \mathbf{( 1 0 ^ { \mathbf { 5 } }} \\
\text { cells/mL) }\end{array}$ & Cell Disruption (\%) \\
\hline Control-algae in & 20.25 & -- \\
\hline water & & \\
\hline $\mathbf{1}$ & 15.50 & 23.46 \\
\hline $\mathbf{2}$ & 10.25 & 49.38 \\
\hline $\mathbf{3}$ & 7.50 & 62.96 \\
\hline $\mathbf{4}$ & 12.75 & 37.04 \\
\hline $\mathbf{5}$ & 15.75 & 22.22 \\
\hline $\mathbf{6}$ & 12.75 & 37.04 \\
\hline $\mathbf{7}$ & 9.00 & 55.56 \\
\hline $\mathbf{9}$ & 10.00 & 50.62 \\
\hline $\mathbf{9}$ & 4.50 & 77.78 \\
\hline $\mathbf{1 0}$ & 6.25 & 69.14 \\
\hline $\mathbf{1 1}$ & 13.50 & 33.33 \\
\hline $\mathbf{1 2}$ & 14.50 & 28.40 \\
\hline
\end{tabular}

The addition of the ultrasonication method to the modified Bligh and Dyer method increased cell disruption efficiency by $44.45 \%$ and $34.56 \%$ for d-limonene and p-cymene, respectively. This further indicates the successful application of ultrasound-assisted modified Bligh and Dyer combined extraction method in the present study. As cited in literature, the ultrasonication method is a promising application for oil extraction (Kumar et al., 2017). However, the choice of solvent and its subsequent physical properties greatly influence the 
effectiveness of the ultrasonication process. Previously discussed, solvents such as chloroform and methanol fair better than the terpenes used in the present study. Therefore, applying another extraction technique provides another opportunity for cell disruption. The initial ultrasonication process ruptured the cell walls to a certain degree and the subsequent modified Bligh and Dyer provided the opportunity of a terpene to further penetrate the cell wall.

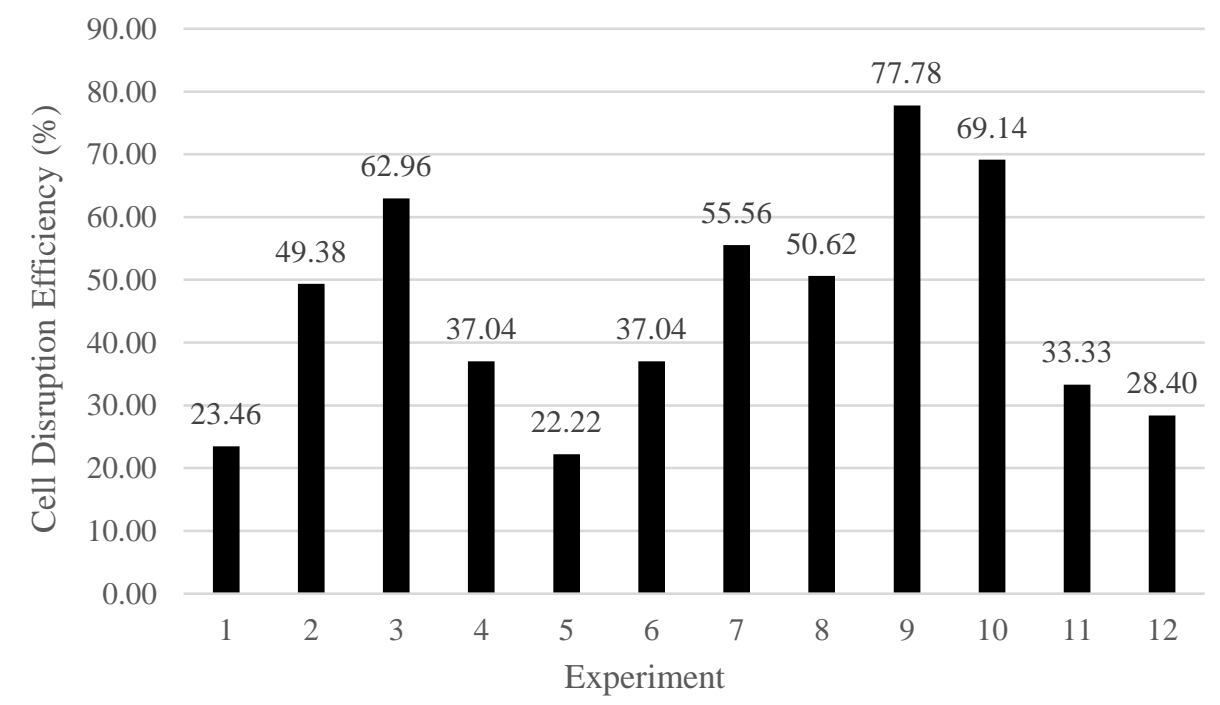

Figure 4.5 Cell disruption of different oil extraction methods

\subsubsection{Algal Oil Extraction: Post-Fermentation}

Examination of using virgin algal biomass versus using the remaining algal biomass recovered following fermentation can indicate that the latter has resulted in a higher oil yield. The two-step process of oil extraction using ultrasonication and the green, modified Bligh and Dyer method with d-limonene resulted in the highest oil yield of $46.27 \%$ when compared to the other processes as previously discussed for virgin algal biomass. These optimal conditions of ultrasonication followed by the green, modified Bligh and Dyer method were applied to algal biomass recovered following fermentation. This investigation was carried out in duplicates to 
ensure repeatability and validity. Raw data of this investigation can be found in Appendix E. As seen in Table 4.14, the recovered algal biomass resulted in an average oil yield of $65.04 \%$ which is approximately $18.77 \%$ greater than the oil yield that resulted from using virgin algal biomass.

Table 4.14 Oil yield from virgin algal biomass and recovered algal biomass using the combined oil extraction process using d-Limonene

\section{Experiment}

1. Virgin algal biomass

2. Recovered algal biomass after fermentation

\section{Oil Yield (\%)}

46.27

65.04

The prime difference between the virgin algal biomass and the recovered algal biomass is that the former biomass has not been exposed to any treatments. On the contrary, the latter has been subjected to a variety of different treatments including chemical, thermal, and enzymatic. During this process, the microalgal cell wall is broken down which can be advantageous for the following two reasons 1) disintegrates the lignocellulosic biomass to expose sugars necessary for bio-alcoholic fuel production and 2) provides accesses to the lipids in the interior of the cells (Liang et al., 2012). Although the disintegration of the cell wall does provide access to the lipids, it is not sufficient enough to create an adequate solvent-lipid complex which can diffuse out of the cell membrane. Applying specific oil extraction processes can further facilitate the lipid extraction. Therefore, a solvent, in this case a terpene, is still required to create a solvent-lipid complex in order to sufficiently release lipids.

As a result of pre-treatments, the cell wall of the recovered algal biomass is already disintegrated thus allowing the exposure and relatively easier access to the lipids in the interior of the cell. Consequently, the same oil extraction processes will result in a higher oil yield of the recovered algal biomass. On the contrary, since the cell wall of the virgin algal biomass is essentially untouched and remains intact, it is much more difficult to breakdown, expose, and 
release the lipids. The same amount of energy generated through oscillating waves from ultrasonication and the chemical energy through the green, modified Bligh and Dyer method is required to breakdown the cell wall and expose the lipids as well as create the solvent-lipid complex to be diffused through the cell membrane. The recovered algal biomass has an advantage as its cell wall has already been disintegrated and the lipids have been exposed to a certain degree. Therefore, the energy utilized in the two-step oil extraction process is directed primarily towards lipid release.

As discussed by Amit et al. (2011), the fermentation process does not affect the fatty acid profile and does not degrade lipid quality. It has been assessed that carrying out the fermentation procedure prior to lipid extraction can be beneficial. The fermentation process results in the formation of peptides that have antioxidant characteristics which can prevent lipid oxidation (Amit et al., 2011 and Ruthu et al., 2014). During lipid oxidation, the quality of lipids can deteriorate due to the development of radicals. Such radicals effectively steal electrons from the lipid cell membrane and thereby causing cell damage (Amit et al., 2011 and Ruthu et al., 2014). Consequently, the formation and presence of peptides can be seen as a positive influence upon oil yield as the recovered algal biomass post-fermentation resulted in a higher oil yield. On the contrary, the virgin algal biomass resulted in an oil yield which was $18.77 \%$ less than the postfermentation sample. The lower recovery rate can perhaps also be attributed to the lack of peptides and the subsequent formation of radicals. Thus, causing potential lipid deterioration, cell damage and consequently a decrease in lipid availability for extraction.

Another important distinguishing characteristic of the recovered algal biomass following fermentation is that it has not only been exposed to the enzymatic pre-treatment but also the enzyme-secreting Clostridia fusant $\mathrm{CbCt}$ during the fermentation process. Enzymatic cell 
disruption processes have become one of the most commonly used techniques when combined with solvent extraction (Sierra et al., 2017). It is an advantageous approach because it incorporates highly selective disruption that permits the extraction of the desired products, mild reactions, and the absence of energy intensive drying steps (Sierra et al., 2017; Zhang et al., 2018). Additionally, enzymatic pre-treatments are used in combination with solvents to breakdown the cell wall, release lipid bodies from the cellular structure and separate the lipids from the protein/lipid matrix (Sierra et al., 2017). Due to the high costs of enzymes, it has been recognized that improving enzymatic hydrolysis treatments is necessary in order to promote efficiency (Zhang et al., 2018).

Liang et al. (2012) reported an oil extraction yield of $49 \%$ for Chlorella vulgaris through ultrasonication followed by enzymatic treatment using snailase. The present study reported an oil extraction yield of $46.27 \%$ using virgin algal biomass subjected to the two-step extraction process of ultrasonication and direct-solvent using d-limonene. The enzyme assisted process resulted in a higher oil extraction yield. When examining the recovered algal biomass, the oil extraction yield of $65.04 \%$ was achieved when subjected to the same two-step extraction process. This is $16.04 \%$ greater than what was reported by Liang et al. (2012). In both instances, enzymatic treatment was applied. Liang et al. (2012) utilized snailase whereas the present study utilized cellulase and $\beta$-glucosidase during the pre-treatment of the algal biomass to break down the cellulose and hemicellulose structures for sugar release. Snailase is a complex that is comprised of a variety of different enzymes including cellulase, hemicellulase, galactase, and $\beta$ glucosidase (Liang et al., 2012). Based on this, it would be sufficient to assume that the oil extraction yield of both investigations should be similar. However, the oil extraction yield achieved in the present study is $16.04 \%$ greater. This can be accounted for by the utilization of 
Clostridia fustant $\mathrm{CbCt}$. This novel fused bacterium produces similar enzymes to that of snailase which essentially work towards breaking down the cell wall, exposing sugars and lipids necessary for biofuel production (Begum and Dahman, 2015). Therefore, this can clearly indicate the suitability and versatility of using a Clostridia fusant during the biofuel production process. The Clostridia fusant, $\mathrm{CbCt}$, was able to achieve a higher biobutanol concentration, compared to single strains, and also improve oil extraction yields post-fermentation. This investigation of using virgin versus recovered algal biomass post-fermentation clearly indicates the overall advantageous role of using fused bacteria strains during the algal biofuel production process. The use of this fusant increases the strength of the enzymatic pre-treatment with the addition of enzymes at no added cost and improves enzymatic hydrolysis efficiency as indicated by Zhang et al. (2018). Therefore, using Clostridia fusants in algal biorefinery applications can be highly economical and efficient. 


\section{CHAPTER 5: \\ CONCLUSIONS AND RECOMMENDATIONS}

\subsection{Conclusions}

The present study focused on enhancing the downstream production of algal-based biofuel production. It was comprised of two main parts which incorporated the evaluation of using novel fused Clostridia sp., $C a C t$ and $C b C t$ on biobutanol production and the evaluation of terpenes as a bio-based alternative solvent for algae oil extraction. Furthermore, the present study also provided rationale for choosing algae as a prime feedstock choice for biofuel production. Algae offer a variety of superior characteristics when compared to first- and second-generation agricultural feedstocks. Having been in its early and developmental stage, third-generation biofuels, comprised of algae feedstock, still require continued research to facilitate improvement.

The protoplast fusion technique applied in this study resulted in the fusants of $\mathrm{CaCt}$ and $C b C t$. The fusant strains were subjected to UV radiation to improve butanol tolerance and thermal stability. Subsequently, the butanol tolerance increased to $15 \mathrm{~g} / \mathrm{L}$ which is more than what has been reported in literature (13 g/L) (Pfromm et al.., 2010). The fused strains and single Clostridia sp. ( $C a, C b$, and $C t$ ) were used in SSF and SHF processes of algal biomass. It was indicated that the fused strains produced significantly higher biobutanol concentrations when compared to the single stains. The biobutanol concentrations achieved by $\mathrm{CaCt}$ and $\mathrm{CbCt}$ were $7.39 \mathrm{~g} / \mathrm{L}$ and $7.98 \mathrm{~g} / \mathrm{L}$, respectively.

When compared with second-generation feedstocks such as wheat straw, hemp, sugar cane, and switch grass, algal biomass produced lower reducing sugars. This can be attributed to lignocellulosic composition of algae. Although algae possess lower cellulose and hemicellulose concentrations, they are also comprised of lipid content. Lipids are essentially another form of 
energy that can be converted to biofuels. Therefore, algae make up for their lower sugar potential by possessing another biological compound that can be used for biofuel production. Moreover, with the added energy potential, from the lipid concentration, the HHV of algae is higher than that of terrestrial plants.

Ultrasonication and a green, modified Bligh and Dyer approach were applied to extract oil from algae. The present study evaluated the use of terpenes as a bio-based, green solvent as an alternative to traditional and harmful chemicals such as chloroform, methanol, and hexane. Ultrasonication in terpene solvent and the green, modified Bligh and Dyer with terpenes were utilized separately for oil extraction. A combination of these two steps were applied to determine the effect of combining these extraction methods. Ultrasonication with terpenes resulted in a higher oil yield of $38.56 \%$ (d-limonene) and $34.70 \%$ (p-cymene). On the other hand, the green, modified Bligh and Dyer method with terpenes resulted in an oil yield of $26.99 \%$ (d-limonene) and $25.06 \%$ (p-cymene). However, the combination method achieved the highest oil yield of 46.27\% using d-limonene. The combination extraction method of ultrasonication followed by a direct bio-based solvent also greatly influenced the cell disruption efficiency. The greatest cell disruption efficiency was $77.78 \%$ for the combination extraction method. By adding the ultrasonication extraction method to the modified, green Bligh and Dyer extraction method, cell disruption increased by $44.45 \%$ and $34.56 \%$ for d-limonene and p-cymene, respectively.

This study also investigated using the remaining algal biomass recovered after fermentation to determine its influence on the oil yield. This algal biomass was subjected to the same two-step oil extraction process use with virgin algal biomass. The oil extraction yield for the recovered algal biomass post-fermentation was $65.04 \%$. When compared with the virgin algal biomass, this oil recovery rate was $18.77 \%$ greater. Upon further examination, it was 
assessed that the algal biomass used in the fermentation process, was exposed to various pretreatments to breakdown the cell wall to expose the necessary sugars for bio-alcoholic fuel production. The chemical, thermal, and enzymatic pre-treatments coupled with the exposure to the enzyme-secreting Clostridia fusant, $C b C t$, resulted in a disintegrated cell wall and exposed lipid structure. Therefore, the energy utilized in the two-step extraction process was primarily focused on creating the solvent-lipid complex and removing the lipids.

When compared with literature values, the oil yield of using terpenes resulted in lower values. As indicated in this study, traditionally used chemicals such as chloroform, methane and hexane fair better in terms of volatility and chemical affinity towards to lipids. However, this study indicated that terpenes are a promising alternative. It was concluded that the use of a single terpene in ultrasonication resulted in higher oil yields than using a hexane and methanol solvent system.

\subsection{Recommendations}

Future studies for algal-based fermentation can incorporate the utilization of a bioreactor to better simulate industrial practices and procedures. Bioreactors have the beneficial capacity to control and monitor specific parameters of the fermentation procedure and can accommodate higher amounts of biomass. Additionally, such a pilot-scale bioreactor can also evaluate the constant removal of butanol from the fermentation broth in order to allow successful deployment of mutant strains.

Another important element of improving algal-based fermentation is to further investigate mutated strains of Clostridia sp. Although this study has indicated that $C b C t$ has the most potential in terms of producing biobutanol, it is recommended that future studies examine different mutant strains through chemofusion and other genetically modified approaches. 
As this study has indicated, green oil extraction from terpenes is a promising alternative. However, further research and studies are necessary for further evaluation and potential use of terpenes to replace traditional chemicals. Improving oil yield derived from terpenes may require additional parameters such as the modified approach of accelerated solvent extraction (ASE). Cooney et al. (2009) and Cheng et al. (2011) have indicated the potential of this modified approach to improve efficiencies and oil yields however the drawback of this method is its current utilization with traditional chemicals. When applied to this modified approach, traditional chemicals such as chloroform and methanol pose severe health and environmental risks. Therefore, it is recommended that future studies examine and evaluate the ASE method with terpenes. The ASE method has slowly become a common method and is becoming a standardized approached for extraction processes. Scientific and pharmaceutical companies, such as Thermo Fisher, have developed automated machines to carry out the ASE method. Thermo Fisher has developed an automated system called the Dionex that is intended for industrial applications. The automated system uses 50-90\% less solvent than other conventional extraction methods such as the Soxhlet. Furthermore, the extraction processes are faster and more efficient thus allowing for more extractions in a given period of time. Using the Dionex automated system to deploy the ASE method with terpenes would be the next step to improve algal oil extraction and to assess the commercial viability of terpenes at the commercial scale. 


\section{APPENDICES}

\section{Appendix A:}

\section{Chemicals}

Table A1: List of all chemicals used in present study

\begin{tabular}{|c|c|c|}
\hline Product & Company & Catalogue Number \\
\hline Glucose & Sigma-Aldrich (St. Louis, MO) & G8769 \\
\hline Xylose & Sigma-Aldrich (St. Louis, MO) & $\mathrm{X} 1500$ \\
\hline Galactose & Sigma-Aldrich (St. Louis, MO) & G0750 \\
\hline Arabinose & Sigma-Aldrich (St. Louis, MO) & A3256 \\
\hline Mannose & Sigma-Aldrich (St. Louis, MO) & M6020 \\
\hline d-biotin & Sigma-Aldrich (St. Louis, MO) & B4501 \\
\hline PABA & Sigma-Aldrich (St. Louis, MO) & 6930 \\
\hline Thiamine-HCL & Sigma-Aldrich (St. Louis, MO) & $\mathrm{T} 4625$ \\
\hline $\mathrm{FeSO}_{4} \cdot 4 \mathrm{H}_{2} \mathrm{O}$ & Sigma-Aldrich (St. Louis, MO) & F8048 \\
\hline $\mathrm{MnSO}_{4} \cdot 7 \mathrm{H}_{2} \mathrm{O}$ & Sigma-Aldrich (St. Louis, MO) & M7634 \\
\hline $\mathrm{MgSO}_{4} \cdot 4 \mathrm{H}_{2} \mathrm{O}$ & Sigma-Aldrich (St. Louis, MO) & 63138 \\
\hline $\mathrm{H}_{2} \mathrm{SO}_{4}$ & Sigma-Aldrich (St. Louis, MO) & 339741 \\
\hline $\mathrm{KH}_{4} \mathrm{PO}_{4}$ & Sigma-Aldrich (St. Louis, MO) & GO139 \\
\hline $\mathrm{NaOH}$ & Sigma-Aldrich (St. Louis, MO) & S5881 \\
\hline Poly ethylene glycol (PEG) & Sigma-Aldrich (St. Louis, MO) & $\mathrm{X} 2753$ \\
\hline Casein hydrsolate & Sigma-Aldrich (St. Louis, MO) & 22090 \\
\hline$\left(\mathrm{NH}_{4}\right)_{2} \mathrm{SO}_{4}$ & Sigma-Aldrich (St. Louis, MO) & 778202 \\
\hline Agar & Sigma-Aldrich (St. Louis, MO) & 05038 \\
\hline Cellulase & Sigma-Aldrich (St. Louis, MO) & $\mathrm{C} 2730$ \\
\hline B-glucosidase & Sigma-Aldrich (St. Louis, MO) & G4511 \\
\hline d-limonene & Sigma-Aldrich (St. Louis, MO) & 183164 \\
\hline p-cymene & Sigma-Aldrich (St. Louis, MO) & C121452 \\
\hline Yeast & Sigma-Aldrich (St. Louis, MO) & 70161 \\
\hline Peptone & Sigma-Aldrich (St. Louis, MO) & P5905 \\
\hline Starch & Sigma-Aldrich (St. Louis, MO) & 33615 \\
\hline $\mathrm{NaCl}$ & Sigma-Aldrich (St. Louis, MO) & S7653 \\
\hline Sodium Acetate & Sigma-Aldrich (St. Louis, MO) & W302406 \\
\hline Cysteine hydrochloride & Sigma-Aldrich (St. Louis, MO) & C1276 \\
\hline Glycerol & Sigma-Aldrich (St. Louis, MO) & 56815 \\
\hline $\mathrm{CaCl} 2$ & Sigma-Aldrich (St. Louis, MO) & 100435524 \\
\hline Lysozyme & Sigma-Aldrich (St. Louis, MO) & L6876 \\
\hline Gelatin & Sigma-Aldrich (St. Louis, MO) & 90000708 \\
\hline Casmino acids & Sigma-Aldrich (St. Louis, MO) & S7995 \\
\hline Asparagine & Sigma-Aldrich (St. Louis, MO) & A0884 \\
\hline $\mathrm{K}_{2} \mathrm{HPO}_{4}$ & Sigma-Aldrich (St. Louis, MO) & 322431 \\
\hline Sucrose & Sigma-Aldrich (St. Louis, MO) & S0389 \\
\hline
\end{tabular}




\section{Appendix B:}

UV Mutagenesis

Table B1: Cell Concentration of CaCt fused strain exposed to UV radiation

\begin{tabular}{|l|c|c|}
\hline Time (minutes) & $\begin{array}{l}\text { Cell Count } \mathbf{( 1 0}^{\mathbf{5}} \\
\text { cells/mL) }\end{array}$ & $\begin{array}{c}\text { Cell Count with } \mathbf{1 5 g} / \mathbf{L} \\
\text { Butanol (10 }\end{array}$ \\
\hline $\mathbf{0}$ & 26 & 1.5 \\
\hline $\mathbf{5}$ & 24.5 & 6.25 \\
\hline $\mathbf{1 0}$ & 17.75 & 11.25 \\
\hline $\mathbf{1 5}$ & 12.0 & 9.5 \\
\hline $\mathbf{2 0}$ & 5.75 & 4 \\
\hline $\mathbf{2 5}$ & 0.5 & 0.25 \\
\hline
\end{tabular}

Table B2: Cell Concentration of $\mathrm{CbCt}$ fused strain exposed to $\mathrm{UV}$ radiation

\begin{tabular}{|l|c|c|}
\hline Time (minutes) & $\begin{array}{l}\text { Cell Count } \mathbf{1 0}^{\mathbf{5}} \\
\text { cells/mL) }\end{array}$ & $\begin{array}{c}\text { Cell Count with } \mathbf{1 5 g} / \mathbf{L} \\
\text { Butanol (10 }\end{array}$ \\
\hline $\mathbf{0}$ & 28.75 & 2.25 \\
\hline $\mathbf{5}$ & 25.75 & 12 \\
\hline $\mathbf{1 0}$ & 21.75 & 16.75 \\
\hline $\mathbf{1 5}$ & 15.25 & 10.5 \\
\hline $\mathbf{2 0}$ & 9.0 & 4.25 \\
\hline $\mathbf{2 5}$ & 1.0 & 0.25 \\
\hline
\end{tabular}




\section{Appendix C:}

\section{Calibration Curves}

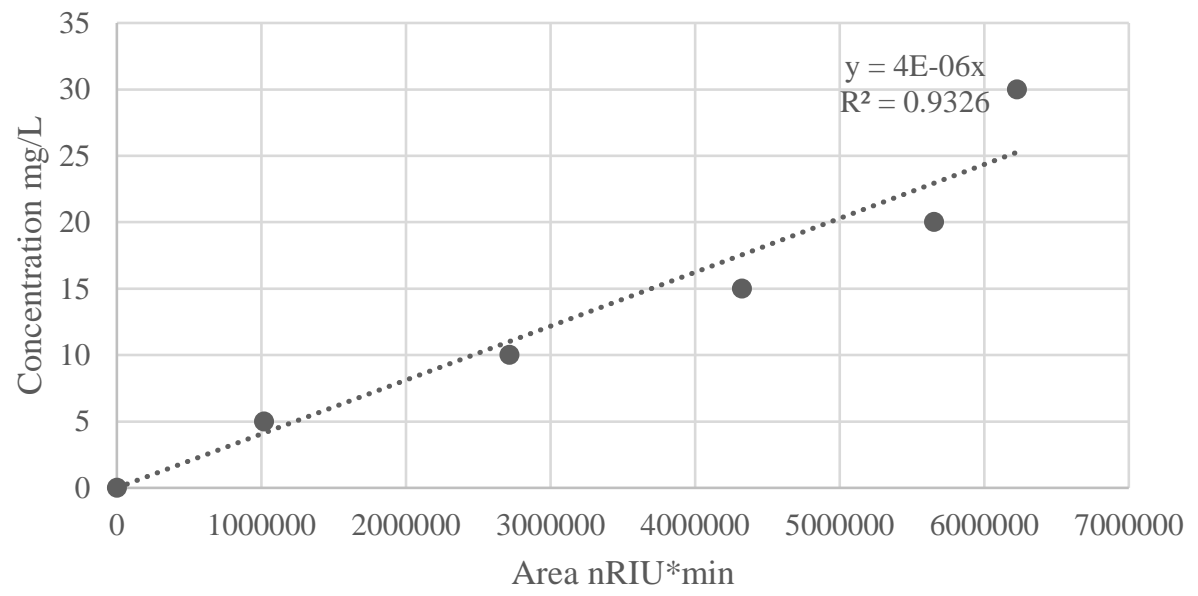

Figure C1: Glucose sugar HPLC calibration curve

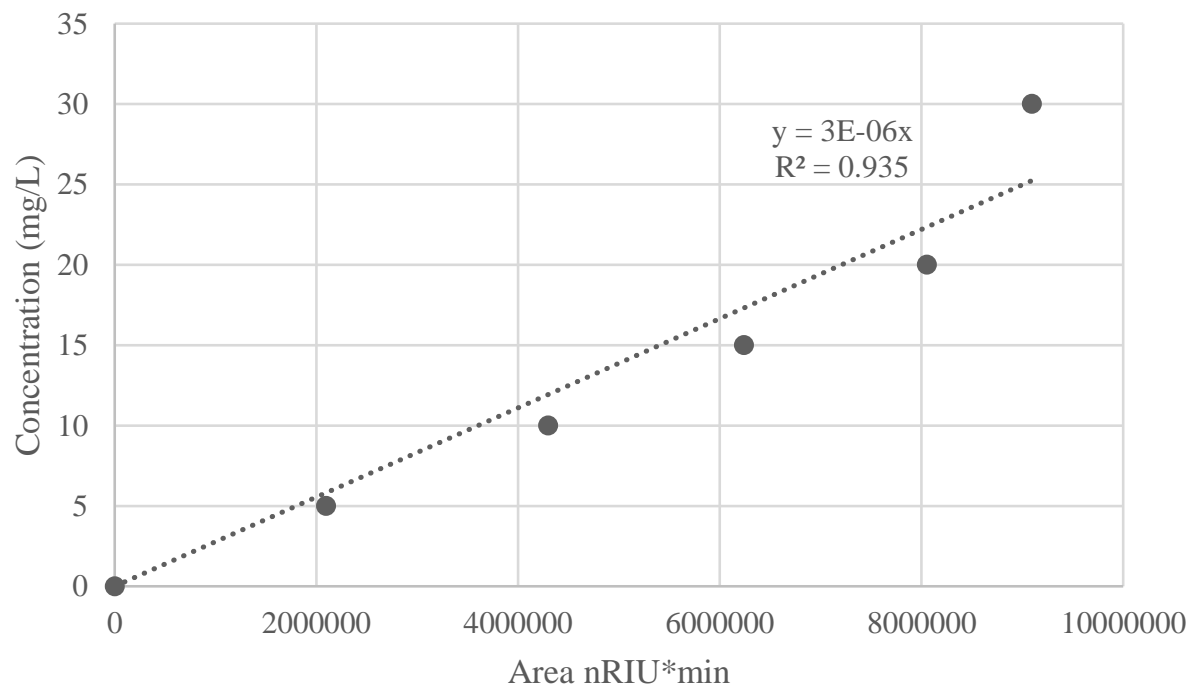

Figure C2: Xylose sugar HPLC calibration curve 


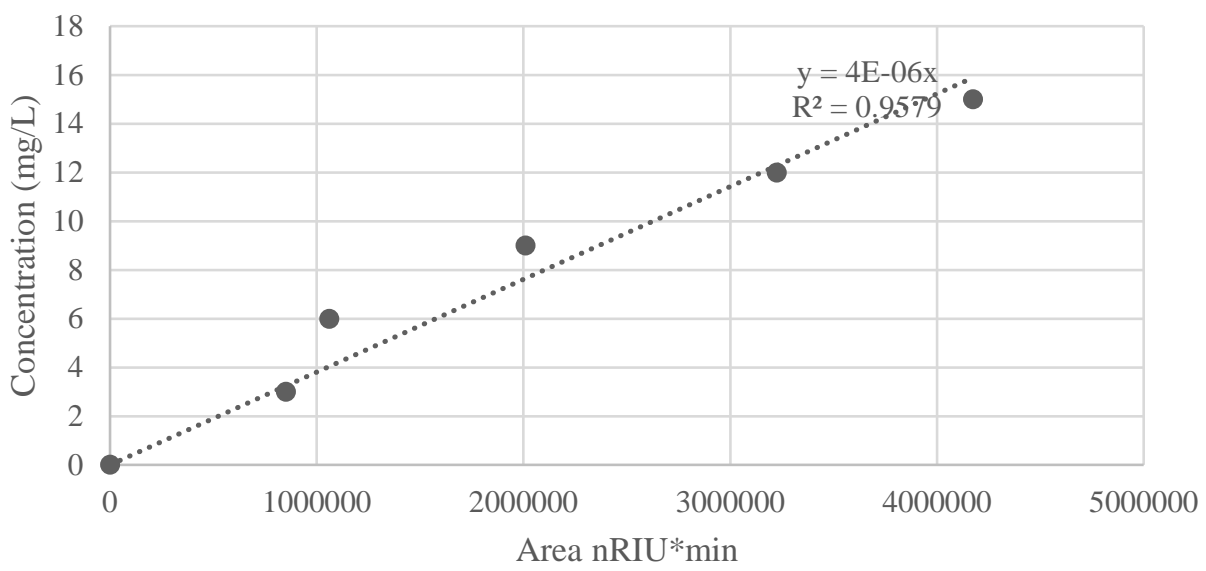

Figure C3: Galactose sugar HPLC calibration curve

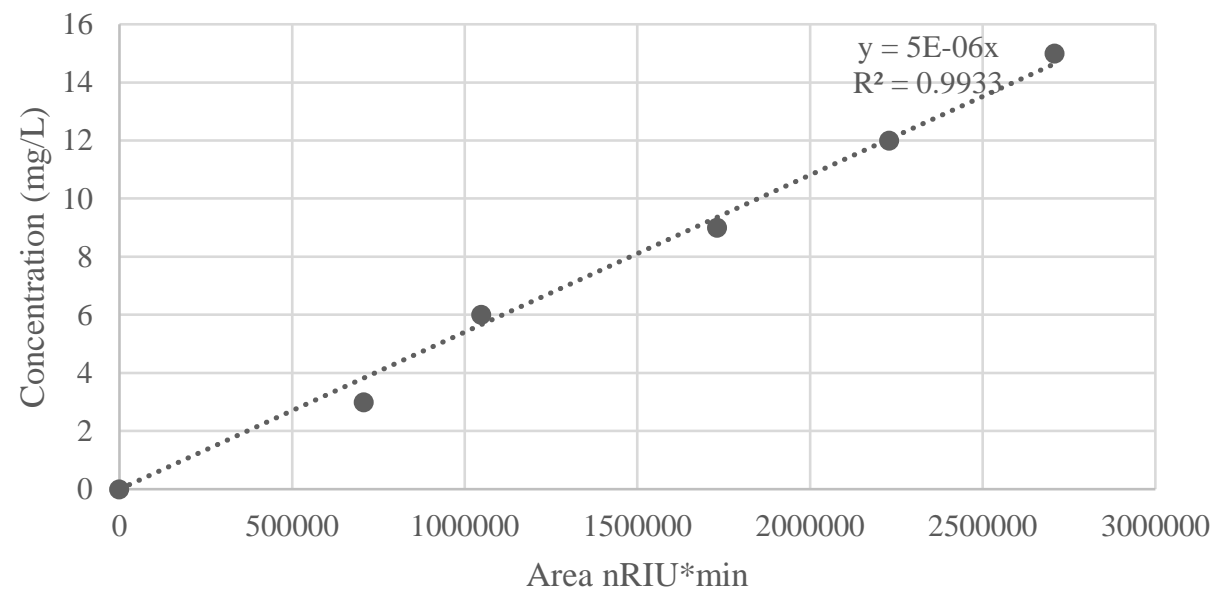

Figure C4: Arabinose sugar HPLC calibration curve 


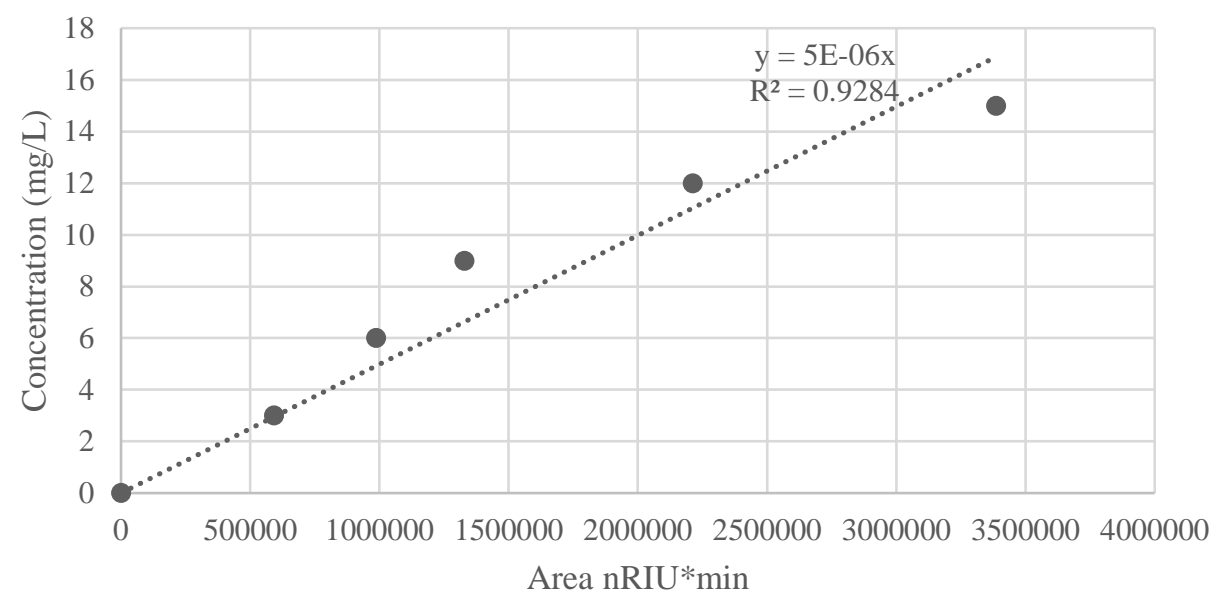

Figure C5: Mannose sugar HPLC calibration curve

Solvent and Acid Calibration Curves

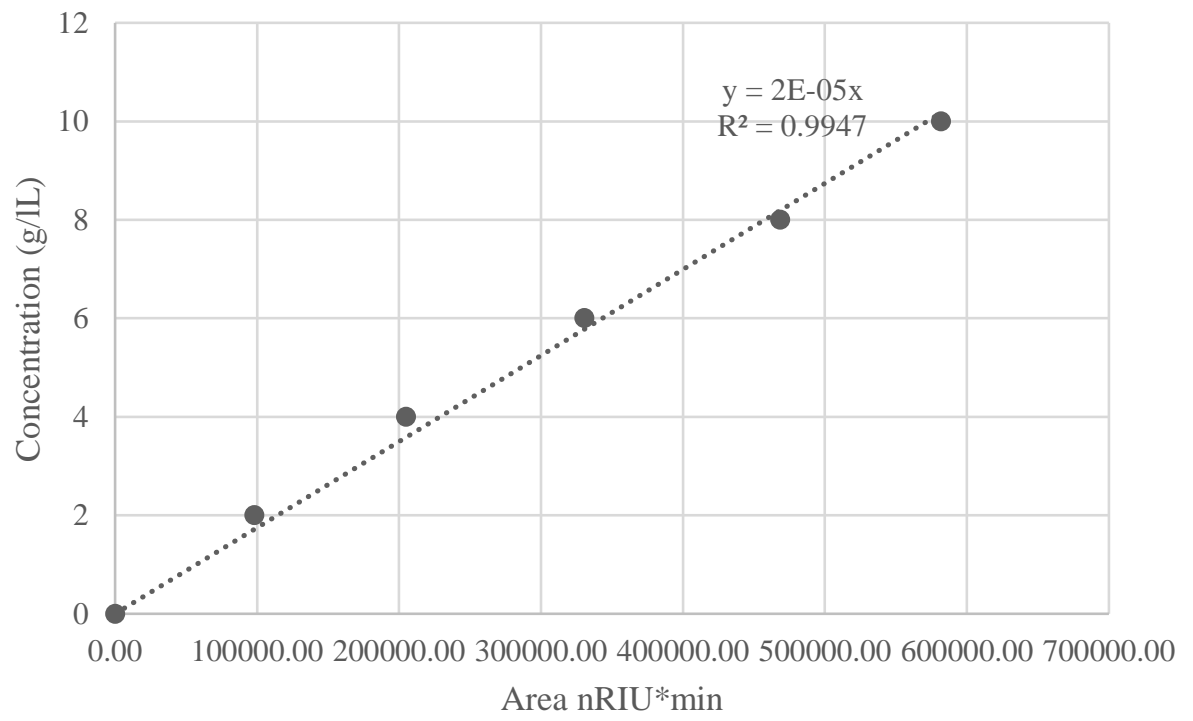

Figure C6: Acetone HPLC calibration curve 


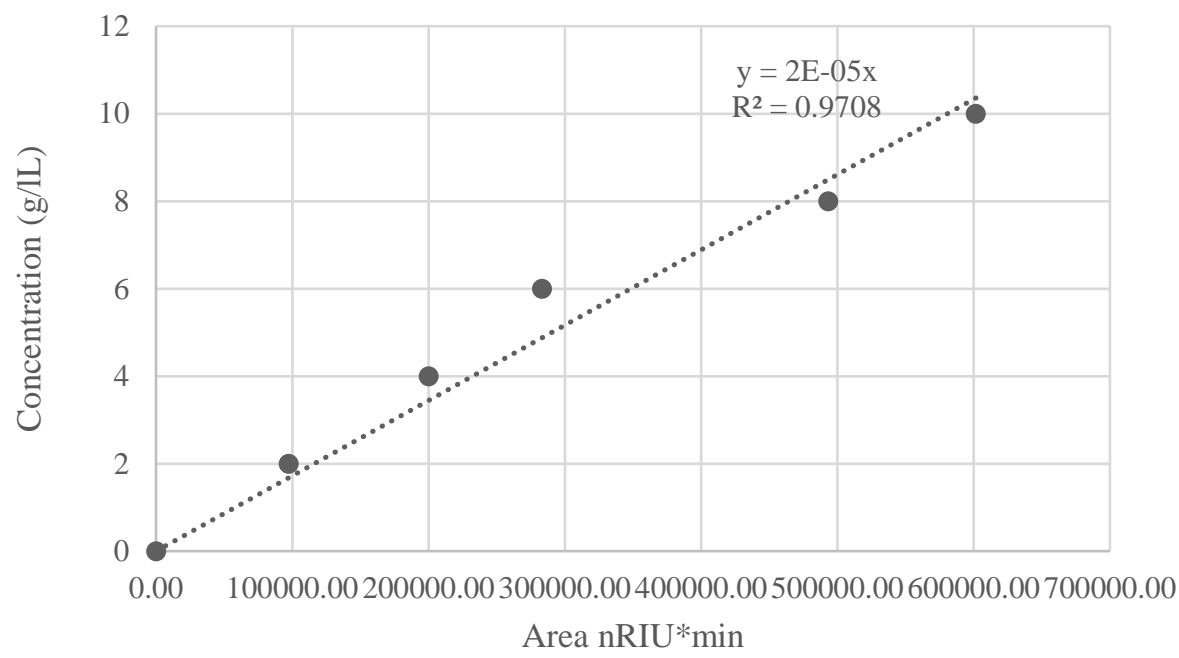

Figure C7: Butanol HPLC calibration curve

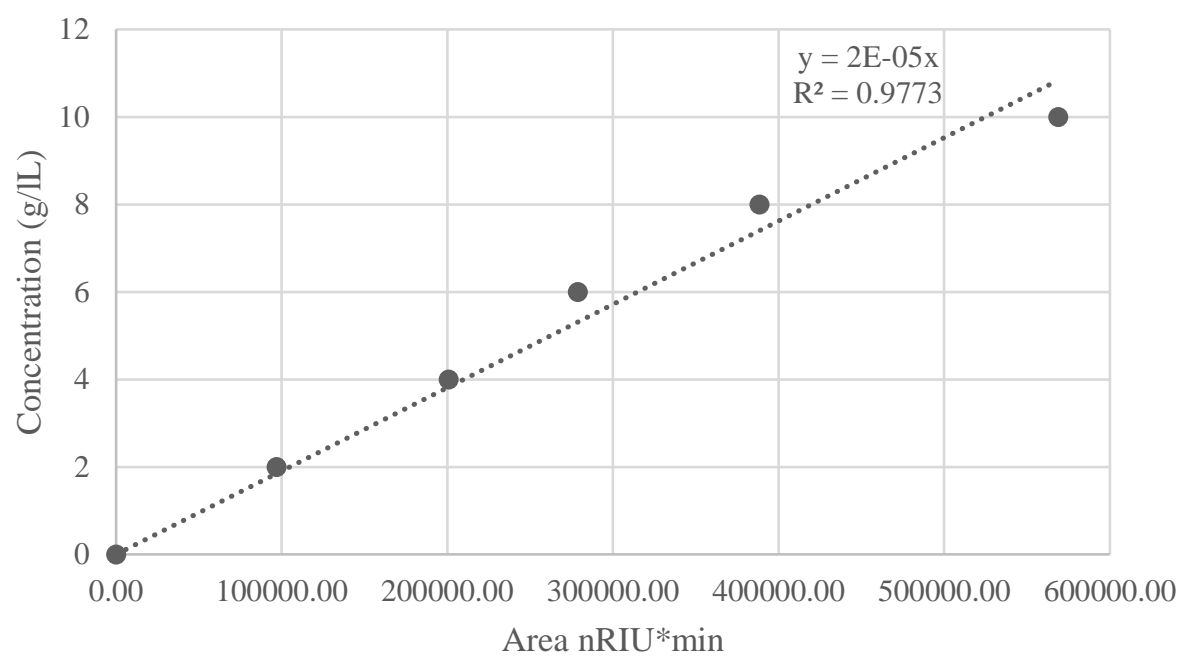

Figure C8: Ethanol HPLC calibration curve 


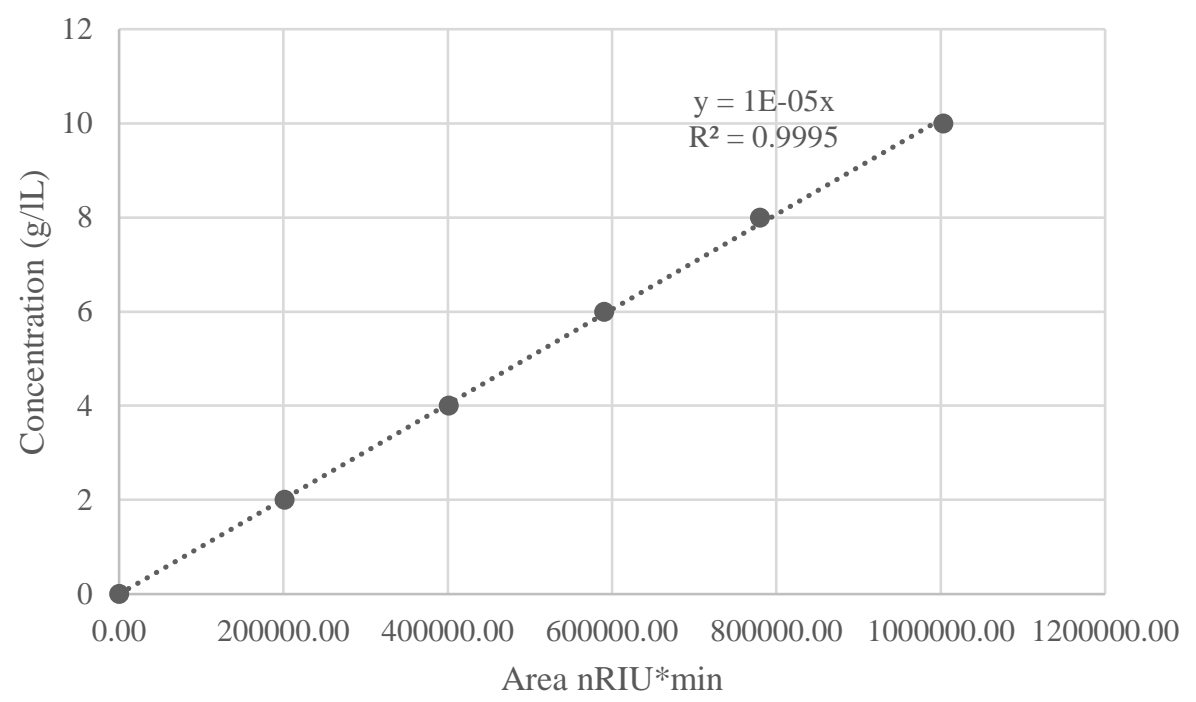

Figure C9: Acetic acid HPLC calibration curve

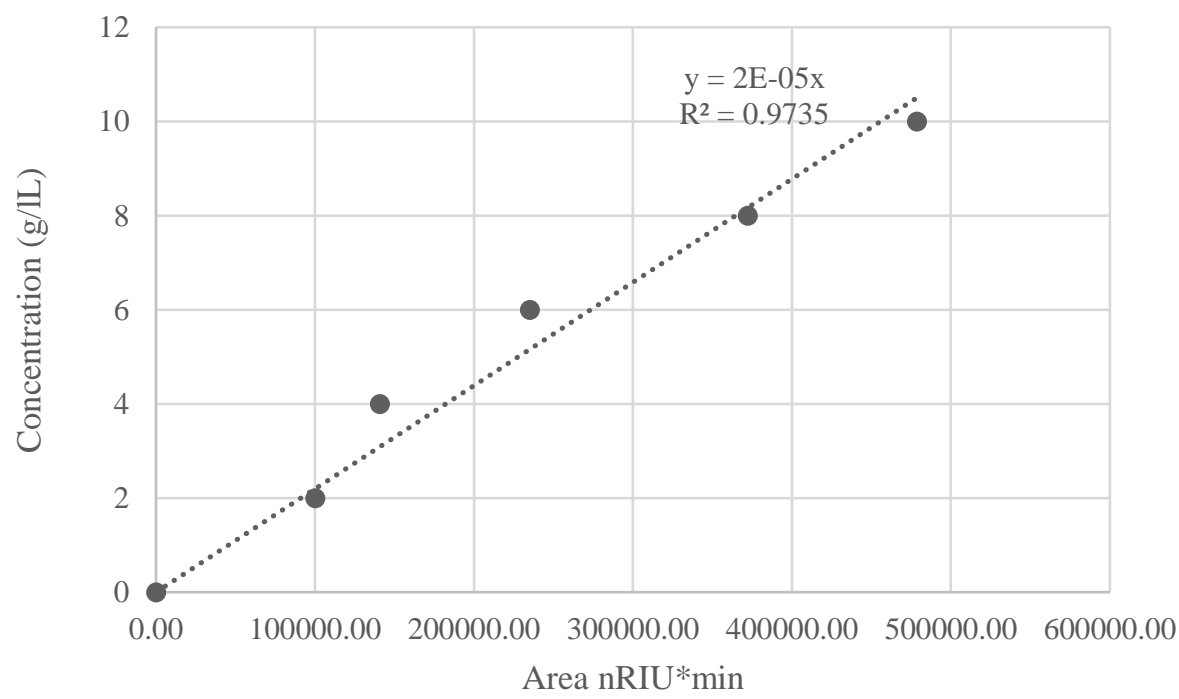

Figure C10: Butyric acid HPLC calibration curve 


\section{Appendix D:}

Fermentation

Table D1: ABE concentration for each fermentation experiment with algal biomass

\begin{tabular}{|l|l|l|r|r|r|}
\hline Experiment & Bacteria & Pre-Treatment & ABE (g/L) & STDEV & \multicolumn{1}{|l|}{ RSD } \\
\hline $\mathbf{1}$ & $\mathrm{CaCt}$ & 1 & 11.47 & 0.01 & 0.11 \\
\hline $\mathbf{2}$ & $\mathrm{CaCt}$ & 2 & 12.11 & 0.01 & 0.10 \\
\hline $\mathbf{3}$ & $\mathrm{CaCt}$ & 3 & 12.60 & 0.08 & 0.65 \\
\hline $\mathbf{4}$ & $\mathrm{CbCt}$ & 1 & 12.29 & 0.01 & 0.08 \\
\hline $\mathbf{5}$ & $\mathrm{CbCt}$ & 2 & 13.43 & 0.05 & 0.35 \\
\hline $\mathbf{6}$ & $\mathrm{CbCt}$ & 3 & 14.02 & 0.01 & 0.09 \\
\hline $\mathbf{7}$ & $\mathrm{Ca}$ & 3 & 9.93 & 0.05 & 0.47 \\
\hline $\mathbf{8}$ & $\mathrm{Cb}$ & 3 & 10.30 & 0.08 & 0.79 \\
\hline $\mathbf{9}$ & $\mathrm{Ct}$ & 3 & 11.00 & 0.004 & 0.04 \\
\hline
\end{tabular}

Pre-treatment 1 incorporated $\mathrm{H}_{2} \mathrm{O}$ with enzymes

Pre-treatment 2 incorporated 1\% Sulfuric Acid

Pre-treatment 3 incorporated $1 \%$ Sulfuric Acid with enzymes

Table D2: Biobutanol concentration of each fermentation experiment

\begin{tabular}{|l|l|l|l|l|r|r|c|}
\hline Experiment & Bacteria & \multicolumn{1}{l|}{ F1 } & \multicolumn{1}{l|}{ F2 } & \multicolumn{1}{l|}{ F3 } & Average & STDEV & RSD \\
\hline $\mathbf{1}$ & $\mathrm{CaCt}$ & 7.11 & 7.01 & 7.10 & 7.07 & 0.04 & 0.64 \\
\hline $\mathbf{2}$ & $\mathrm{CaCt}$ & 7.40 & 7.42 & 7.45 & 7.42 & 0.02 & 0.28 \\
\hline $\mathbf{3}$ & $\mathrm{CaCt}$ & 7.39 & 7.40 & 7.39 & 7.39 & 0.01 & 0.05 \\
\hline $\mathbf{4}$ & $\mathrm{CbCt}$ & 7.41 & 7.43 & 7.44 & 7.43 & 0.01 & 0.17 \\
\hline $\mathbf{5}$ & $\mathrm{CbCt}$ & 7.83 & 7.84 & 7.85 & 7.84 & 0.01 & 0.10 \\
\hline $\mathbf{6}$ & $\mathrm{CbCt}$ & 7.98 & 7.97 & 8.00 & 7.98 & 0.01 & 0.16 \\
\hline $\mathbf{7}$ & $\mathrm{Ca}$ & 5.94 & 5.91 & 5.99 & 5.95 & 0.03 & 0.55 \\
\hline $\mathbf{8}$ & $\mathrm{Cb}$ & 5.90 & 5.96 & 5.97 & 5.94 & 0.03 & 0.52 \\
\hline $\mathbf{9}$ & $\mathrm{Ct}$ & 6.03 & 6.04 & 6.00 & 6.02 & 0.02 & 0.28 \\
\hline
\end{tabular}


Table D3: Total sugar concentrations for each day for each fermentation experiment

\begin{tabular}{|l|l|l|l|l|l|c|c|}
\hline Experiment & Bacteria & $\begin{array}{l}\text { Pre- } \\
\text { Treatment }\end{array}$ & $\begin{array}{l}\text { Day } \\
\mathbf{1}(\mathbf{g} / \mathbf{L})\end{array}$ & $\begin{array}{l}\text { Day 2 } \\
(\mathbf{g} / \mathbf{L})\end{array}$ & $\begin{array}{l}\text { Day 3 } \\
(\mathbf{g} / \mathbf{L})\end{array}$ & $\begin{array}{l}\text { Day 4 } \\
(\mathbf{g} / \mathbf{L})\end{array}$ & $\begin{array}{l}\text { Day 5 } \\
(\mathbf{g} / \mathbf{L})\end{array}$ \\
\hline $\mathbf{1}$ & $\mathrm{CaCt}$ & 1 & 19.39 & 16.52 & 13.76 & 10.58 & 9.18 \\
\hline $\mathbf{2}$ & $\mathrm{CaCt}$ & 2 & 17.01 & 13.70 & 12.36 & 8.10 & 8.30 \\
\hline $\mathbf{3}$ & $\mathrm{CaCt}$ & 3 & 14.87 & 11.89 & 9.69 & 7.46 & 6.80 \\
\hline $\mathbf{4}$ & $\mathrm{CbCt}$ & 1 & 17.92 & 14.95 & 12.56 & 8.82 & 7.33 \\
\hline $\mathbf{5}$ & $\mathrm{CbCt}$ & 2 & 16.10 & 13.12 & 10.30 & 7.65 & 6.57 \\
\hline $\mathbf{6}$ & $\mathrm{CbCt}$ & 3 & 13.58 & 10.94 & 8.13 & 6.55 & 5.97 \\
\hline $\mathbf{7}$ & $\mathrm{Ca}$ & 3 & 21.49 & 21.76 & 19.38 & 16.91 & 15.83 \\
\hline $\mathbf{8}$ & $\mathrm{Cb}$ & 3 & 21.35 & 21.46 & 18.87 & 16.81 & 15.28 \\
\hline $\mathbf{9}$ & $\mathrm{Ct}$ & 3 & 20.44 & 20.56 & 17.95 & 16.76 & 15.09 \\
\hline
\end{tabular}

Pre-treatment 1 incorporated $\mathrm{H}_{2} \mathrm{O}$ with enzymes

Pre-treatment 2 incorporated 1\% Sulfuric Acid

Pre-treatment 3 incorporated 1\% Sulfuric Acid with enzymes

Table D4: ABE yield for each fermentation experiment

\begin{tabular}{|l|l|l|l|l|}
\hline Experiment & $\begin{array}{l}\text { ABE } \\
\text { Yield } \\
\text { (g/g) }\end{array}$ & Acetone Yield $(\mathbf{g} / \mathbf{g})$ & Butanol Yield $\mathbf{( g / g )}$ & Ethanol Yield $(\mathbf{g} / \mathbf{g})$ \\
\hline $\mathbf{1}$ & 0.50 & 0.15 & 0.31 & 0.05 \\
\hline $\mathbf{2}$ & 0.49 & 0.14 & 0.30 & 0.05 \\
\hline $\mathbf{3}$ & 0.51 & 0.16 & 0.29 & 0.04 \\
\hline $\mathbf{4}$ & 0.51 & 0.17 & 0.30 & 0.05 \\
\hline $\mathbf{5}$ & 0.49 & 0.15 & 0.29 & 0.05 \\
\hline $\mathbf{6}$ & 0.52 & 0.18 & 0.30 & 0.04 \\
\hline $\mathbf{7}$ & 0.43 & 0.14 & 0.26 & 0.04 \\
\hline $\mathbf{8}$ & 0.42 & 0.13 & 0.25 & 0.04 \\
\hline $\mathbf{9}$ & 0.43 & 0.13 & 0.25 & 0.04 \\
\hline
\end{tabular}

Pre-treatment 1 incorporated $\mathrm{H}_{2} \mathrm{O}$ with enzymes

Pre-treatment 2 incorporated 1\% Sulfuric Acid

Pre-treatment 3 incorporated 1\% Sulfuric Acid with enzymes 


\section{Appendix E:}

Oil Extraction

Table E1: Raw data for each ultrasonication oil extraction with algal biomass

\begin{tabular}{|l|c|c|c|c|c|c|}
\hline Experiment & \multicolumn{2}{|c|}{ Oil Extracted (g) } & Average Oil (g) & STDEV & \multicolumn{1}{l|}{ RSD } & Oil Yield (\%) \\
\hline $\mathbf{1}$ & 0.05 & 0.03 & 0.04 & 0.01 & 0.35 & 5.14 \\
\hline $\mathbf{2}$ & 0.25 & 0.23 & 0.24 & 0.01 & 0.06 & 30.85 \\
\hline $\mathbf{3}$ & 0.28 & 0.32 & 0.30 & 0.02 & 0.09 & 38.56 \\
\hline $\mathbf{4}$ & 0.23 & 0.22 & 0.225 & 0.01 & 0.03 & 28.92 \\
\hline $\mathbf{5}$ & 0.04 & 0.03 & 0.035 & 0.01 & 0.20 & 4.50 \\
\hline $\mathbf{6}$ & 0.13 & 0.15 & 0.14 & 0.01 & 0.10 & 17.99 \\
\hline $\mathbf{7}$ & 0.25 & 0.29 & 0.27 & 0.02 & 0.11 & 34.70 \\
\hline $\mathbf{8}$ & 0.19 & 0.21 & 0.20 & 0.01 & 0.07 & 25.71 \\
\hline $\mathbf{9}$ & 0.01 & 0.009 & 0.01 & 0.001 & 0.06 & 1.22 \\
\hline
\end{tabular}

Table E2: Raw data for each modified, green Bligh and Dyer oil extraction with algal biomass

\begin{tabular}{|l|c|c|c|c|c|c|}
\hline Experiment & \multicolumn{2}{|c|}{ Oil Extracted (g) } & Average Oil (g) & STDEV & \multicolumn{1}{l|}{ RSD } & Oil Yield (\%) \\
\hline $\mathbf{1}$ & 0.20 & 0.22 & 0.21 & 0.005 & 0.07 & 26.99 \\
\hline $\mathbf{2}$ & 0.19 & 0.20 & 0.197 & 0.0025 & 0.04 & 25.06 \\
\hline
\end{tabular}

Table E3: Raw data for combination ultrasound and modified, green Bligh and Dyer oil extraction with algal biomass

\begin{tabular}{|l|c|c|c|c|c|c|}
\hline Experiment & \multicolumn{2}{|c|}{ Oil Extracted (g) } & Average Oil (g) & STDEV & RSD & Oil Yield (\%) \\
\hline $\mathbf{1}$ & 0.35 & 0.37 & 0.36 & 0.01 & 0.04 & 46.27 \\
\hline $\mathbf{2}$ & 0.30 & 0.32 & 0.31 & 0.01 & 0.05 & 39.85 \\
\hline
\end{tabular}

Table E4: Raw data for oil extraction post-fermentation

\begin{tabular}{|c|c|c|c|c|c|c|}
\hline Experiment & $\begin{array}{l}\text { Oil } \\
\text { Extr } \\
\text { (g) }\end{array}$ & cted & $\begin{array}{l}\text { Average } \\
\text { Oil (g) }\end{array}$ & STDEV & RSD & $\begin{array}{l}\text { Oil Yield } \\
(\%)\end{array}$ \\
\hline 1 & 2.64 & 2.42 & 2.53 & 0.11 & 0.04 & 65.04 \\
\hline
\end{tabular}




\section{REFERENCES}

Abraham, R., Barrow, C., \& Puri, M. (2013). Relationship to reducing sugar production and scanning electron microscope structure to pretreated hemp hurd biomass (Cannabis sativa). Biomass and Bioenergy, 58, 180-187.

Adams, F., Albert-Vian, M., Peltier, G., \& Chemat, F. (2012). “'Solvent-free” ultrasoundassisted extraction of lipids from fresh microalgae cells: A green, clean and scalable process, 114(1), 457-465.

Adeniyi, O. M., Azimov, U., \& Burluka, A. (2018). Algae biofuel: Current status and future applications. Renewable and Sustainable Energy Reviews, 90, 316-335.

Agbor, V. B., Cicek, N., Sparling, R., Berlin, A., \& Levin, D. B. (2011). Biomass pretreatment: Fundamentals toward application, Biotechnology Advances, 29(6), 675-685.

Ahlgren, S., \& Di Lucia, L. (2016). Indirect land use changes of biofuel production - a review of modelling efforts and policy developments in the European Union. Biotechnology for Biofuels, 9(1), 1-10.

Akinosho, H.O., Yee, K., Close, D., \& Ragauskas, A.J. (2014). The emergence of Clostridium thermocellum as a high utility candidate for consolidated bioprocessing applications. Front. Chem.

Al hattab, M, Ghaly A, Hammoud A (2015) Microalgae Harvesting Methods for Industrial Production of Biodiesel: Critical Review and Comparative Analysis. J Fundam Renewable Energy Appl 5:154. Alonso, D., Wettstein, S., \& Dumesic, J. (2013). ChemInform Abstract: Bimetallic Catalysts for Upgrading of Biomass to Fuels and Chemicals. Cheminform, 44(12)

Amin, S. (2009). Review on biofuel oil and gas production processes from microalgae. Energy Conversion and Management, 50(7), 1834-1840.

Amit, K.R., Jini,R., Swapna, H.C., Sachindra, N.M., Bhaskar, N., Baskaran. V. (2011). Application of native lactic acid bacteria (LAB) for fermentative recovery of lipids and proteins from fish processing wastes: Bioactivities of fermentation products. Journal of Aquatic Food Production Technocology, 20,32-44

Ashokkumar, M.,Leong, T. \& Kentish, S. (2011). The fundamentals of power ultrasound - A review. Acoustics Australia. 39. 
Basu, P. 2010. Biomass Gasification and Pyrolysis: Practical Design and Theory. United States: Academic Press.

Begum, S., and Dahman, Y. (2015). Enhanced biobutanol production using novel clostridial fusants in simultaneous saccharification and fermentation of green renewable agriculture residues. Biofuels, Bioproducts, and Biorefining, 9, 529-544.

Bhardwaj, G., Cameotra, S.S., \& Chopra, H.K. (2015). Utilization of Oil Industry Residues for the Production of Rhamnolipids by Pseudomonas indica. Journal of Surfactants and Detergents, 18, 887-893.

Buday, Z., Linden, J. C., \& Karim, M. N. (1990). Improved acetone-biobutanol fermentation analysis using subambient HPLC column temperature. Enzyme and microbial technology, 12(1), 24-27.

Burlew, S.J. (1976). Algae Culture from Laboratory to Pilot Plant. Washington, D.C., United States: Carnegie Institution of Washington Publication

Bowring. S., and Morris, J.G. (1985). Mutagenesis of Clostridium acetobutylicum. Journal of Applied Microbiology, 58(6), 577-584.

Breil, C., Abert Vian, M., Zemb, T., Kunz, W., \& Chemat, F. (2017). "Bligh and Dyer" and Folch Methods for Solid-Liquid-Liquid Extraction of Lipids from Microorganisms. Comprehension of Solvatation Mechanisms and towards Substitution with Alternative Solvents. International journal of molecular sciences, 18(4), 708. doi:10.3390/ijms 18040708

Bulkowska, K., Gusiatin, Z. M., Klimiuk, E., Pawłowski, A., \& Pokoj, T. (2016). Biomass for biofuels. London, England: CRC Press.

Bussemaker, M. J., \& Zhang, D. (2013). Effect of Ultrasound on Lignocellulosic Biomass as a Pretreatment for Biorefinery and Biofuel Applications. Industrial \& Engineering Chemistry Research, 52(10), 3563-3580. doi:10.1021/ie3022785

Canada's EcoFicscal Commission. (2016). Course Correction: It's Time to Rethink Canadian Biofuel Policies. Toronto, Canada

Capuzzo, A., Maffei, M.E., \& Occhipinti, A. (2013). Supercritical Fluid Extraction of Plant Flavors and Fragrances. Molecules.

Cheng, Hai-Hsuan \& Whang, Liang-Ming \& Chan, Kun-Chi \& Chung, Man-Chien \& Wu, Shu- 
Hsien \& Liu, Cheng-Pin \& Tien, Shih-Yuan \& Chen, Shan-Yuan \& Chang, Jo-Shu \& Lee, Wen-Jhy. (2014). Biological butanol production from microalgae-based biodiesel residues by Clostridium acetobutylicum. Bioresource technology. 184. 10.1016/j.biortech.2014.11.017.

Cherian, A. (2015). Energy and global climate change: Bridging the sustainable development divide. Sussex, England: Wiley Blackwell.

Cho, H., Oh, Y., Park, S., Lee, J.W., \& Park, J. (2013). Effects of enzymatic hydrolysis on lipid extraction from Chlorella vulgaris.

Choi, S.P., Nguyen, M.T., \& Sim, S.J. (2011). Enzymatic pretreatment of Chlamydomonas reinhardtii biomass for ethanol production. Bioresource technology, 101 14, 5330-6 .

Choi, S.-A., Lee, J.-S., Oh, Y.-K., Jeong, M.-J., Kim, S., Park, J.-Y., (2014) Lipid extraction from Chlorella vulgaris by molten-salt/ionic-liquid mixtures. Algal Res. 3, 44-48.

Ciriminna, R., Lomeli-Rodriguez, M., Cara, P. D., Lopez-Sanchez, J. A., \& Pagliaro, M. (2015). Limonene: A Versatile Chemical of the Bioeconomy. ChemInform, 46(5). doi:10.1002/chin.201505238

Cooney, M.,Young G., \& Nagle N. (2009) Extraction of Bio-oils from Microalgae, Separation \& Purification Reviews, 38:4, 291-325, DOI: 10.1080/15422110903327919

Czyrnek-Delêtre, M. M., Chiodi, A., Murphy, J. D., \& Gallachóir, B. P. (2016). Impact of including land-use change emissions from biofuels on meeting GHG emissions reduction targets: The example of Ireland. Clean Technologies and Environmental Policy, 18(6), $1745-1758$.

Dabbs, D.M., Mulders, N., \& Aksay, I.A. (2006). Solvothermal removal of the organic template from L3 ("sponge") templated silica monoliths. Journal of Nanoparticle Research, 8, 603614.

Demirbas, A. (2009). Biofuels: Securing the Planet's Future Energy Needs. London, England: Springer.

Demirbas, A., \& Demirbas, M. F. (2011). Importance of algae oil as a source of biodiesel. Energy Conversion and Management, 52(1), 163-170.

Domínguez, M. C., de la Rosa, M., \& Borobio, M. V. (2001). Application of spectrophotometric method for the determination of post-antibiotic effect and comparison with viable counts in agar. Journal of Antimicrobial Chemotherapy, 47(4), 391-398. 
Drira, N., Piras, A., Rosa, A., Porcedda, S., Dhaouadi, H. (2016) Microalgae from domestic wastewater facility's high rate algal pond: lipids extraction, characterization and biodiesel production, Bioresour. Technol. 206.239-244, https://doi.org/10.1016/j.biortech.2016.01.082.

Dürre, P. (2005). Handbook on Clostridia. Boca Raton, Florida: Taylor \& Francis.

Eggert, H., \& Greaker, M. (2014). Promoting second generation biofuels: Does the firstgeneration pave the road? Energies, 7(12), 4430-4445

El-Dalatony, M., Salama, E., Kurade, M., Hassan, S., Oh, S., Kim, S., \& Jeon, B. (2017). Utilization of microalgal biofractions for bioethanol, higher alcohols, and biodiesel production: A Review. Energies, 10(12), 2110.

El-Zaeddi, H., Martínez-Tomé, J., Calín-Sánchez, Á., Burló, F., \& Carbonell-Barrachina, Á.A. (2016). Volatile Composition of Essential Oils from Different Aromatic Herbs Grown in Mediterranean Regions of Spain. Foods.

Energy Centre Netherlands (a). (2019). Wheat Straw (\#3161): Phyllis2 Database. Retrieved from https://phyllis.nl/Biomass/View/3161

Energy Centre Netherlands (b). (2019). Hemp (\#1193): Phyllis2 Database. Retrieved from https://phyllis.nl/Biomass/View/1193

Energy Centre Netherlands (c). (2019). Sugarcane bagasse (\#2806). Phyllis2 Database. Retrieved from https://phyllis.nl/Biomass/View/2806

Energy Centre Netherlands (d). (2019). Switchgrass (\#896). Phyllis2 Database. Retrieved from https://phyllis.nl/Biomass/View/896

Ezeji, T., Qureshi, N., and Blaschek, H.P. (2007). Production of acetone-butanol-ethanol (ABE) in a continuous flow bioreactor using degermed corn and Clostridium beijerinckii. Process Biochemistry, 42(1),34-39

Ezeji, T., Milne, C., Price, N.D., and Blaschek, H.P. (2010). Achievements and perspectives to overcome the poor solvent resistance in acetone and biobutanol-producing microorganisms. Appl. Microbiol. Biotechnol. 85, 1697-1712.

Ezeji, T., Qureshi, N., \& Blaschek, H. P. (2007). Production of acetone-biobutanol-ethanol $(\mathrm{ABE})$ in a continuous flow bioreactor using degermed corn and Clostridium beijerinckii. Process Biochemistry, 42(1), 34-39.

Farmer, G. T. (2014). Modern climate change science: An overview of today's climate change 
science. New York, NY: Springer.

Ferhan, M., \& Dahman, Y. (2016). Novel thermostable clostridial strains through protoplast fusion for enhanced biobutanol production at higher temperature - preliminary study. AIMS Energy, 4(1), 22-36.

Gabriel, C. L. (1928). Butanol fermentation process. Industrial Engineering and Chemistry, 20(1), 1063-1067.

Gille, S. \& Pauly, M. 2012. O-acetylation of plant cell wall polysaccharides. Frontiers in Plant Science 3: 12 .

Gouveia, L. (2011). Microalgae as a feedstock for biofuels. Heidelberg, Germany: Springer.

Government of Canada. (2016). National Inventory Report 1990-2016: Greenhouse Gas Sources and Sinks in Canada. Ottawa, Canada

Grohmann, K., Mitchell, D.J., Himmel, M.E., Dale, B.E. \& Schroeder, H.A. 1989. The role of ester groups in resistance of plant cell wall polysaccharides to enzymatic hydrolysis. Applied Biochemistry and Biotechnology 20/21: 45-61.

Gupta, V. K., \& Tuohy, M. G. (2011). Biofuel technologies: Recent developments. Heidelberg, Germany: Springer.

Günerken, E., D'Hondt, E., Eppink, M. H., Gonzalez, L. G., Elst, K., \& Wijffels, R. H. (2015). Cell disruption for microalgae biorefineries. Biotechnology Advances,33(2), 243-260.

Hallac, B.B. \& Ragauskas, A.J. 2011. Analyzing cellulose degree of polymerization and its relevancy to cellulosic ethanol. Biofuels. Bioproducts and Biorefining 5(2): 215-225.

Halim, R.A., Danquah, M.K., \& Webley, P.A. (2012). Extraction of oil from microalgae for biodiesel production: A review. Biotechnology advances, 30 3, 709-32 .

Harris, J., Viner, K., Champagne, P., \& Jessop, P. G. (2018). Advances in microalgal lipid extraction for biofuel production: A review. Biofuels, Bioproducts and Biorefining, 12(6), 1118-1135. doi:10.1002/bbb.1923

Harmsen, P., Huijgen, W., Bermudez, L. \& Bakker, R. 2010. Literature review of physical and chemical pretreatment processes for lignocellulosic. Biomass: 1-49.

Hatfield, R. \& Vermerris, W. 2001. Lignin formation in plants. The dilemma of linkage specificity. Plant Physiology 126: 1351-1357. 
Helwani, Z., Othman, M.R., Aziz, N., Fernando, W.J.N., \& Kim, J. (2009). Technologies for production of biodiesel focusing on green catalytic techniques:A review. Fuel Processing Technology, 90(1), 1502-1514.

Huang, W.C., Huang, J.D., Kim, Cationic surfactant-based method for simultaneous harvesting and cell disruption of a microalgal biomass, Bioresour. Technol. 149

Ikehata, H., \& Ono, T. (2011). The mechanisms of UV mutagenesis. Journal of radiation research, 52(2), 115-125.

International Energy Agency. (2015). Energy and Climate Change 2015: World Energy Outlook Special Report. Paris, France.International Energy Agency. (2019). Energy Security. Retrieved from https://www.iea.org/topics/energysecurity/

International Energy Agency. (2017). State of Technology Review-Algae Bioenergy. Paris, France.

Jang, Y., Malaviya, A., \& Lee, S.Y. (2012). Acetone-butanol-ethanol production with high productivity using Clostridium acetobutylicum BKM19. Biotechnology and bioengineering, $1106,1646-53$.

Jones, D. T., \& Woods, D. R. (1986). Acetone-biobutanol fermentation revisited. Microbiological reviews, 50(4), 484.

Kao, K. N., \& Michayluk, M. R. (1974). Plant protoplast fusion and growth of intergeneric hybrid cells. Planta, 120(3), 215-227.

Kates M. 1986. Definition and classification of lipids. Techniques of lipidology isolation, analysis, and identification of lipids. Amsterdam: Elsevier Science Publisher

Knothe, G., \& Razon, L. F. (2017). Biodiesel fuels. Progress in Energy and Combustion Science, 58(7), 36-59.

Kumar, S.P.J., Garpalit, V.J., Dash, A., Scholz, P., \& Banerjee, R. (2017). Sustainable green solvents and techniques for lipid extraction from microalgae: A review. Algal Research, 21, 138-147.

Lai, Y.S., De Francesco, F., Aguinaga, A., Parameswaran, P., Rittmann, B.E. (2016) Improving lipid recovery from Scenedesmus wet biomass by surfactant-assisted disruption, Green Chem. 18 1319-1326, https://doi.org/10.1039/ C5GC02159F.

Lee, S.Y., Park, J.H., Jang, S.H., Nielsen, L.K., Kim, J., and Jung, K.S. (2008). Fermentative 
butanolproduction by Clostridia. Biotechnol. Bioeng. 101, 209-228.

Lee, S.Y., Cho, J.M., Chang,Y.K., Y.K. (2017) Cell disruption and lipid extraction for microalgal biorefineries: a review, Bioresour. Technol. 244 1317-1328, https://doi.org/10.1016/j.biortech.2017.06.038.

Leeuwe, M. V., Villerius, L., Roggeveld, J., Visser, R., \& Stefels, J. (2006). An optimized method for automated analysis of algal pigments by HPLC. Marine Chemistry, 102(3-4), 267-275

Levin, D.B., Islam, R., Cicek, N., and Sparling, R. (2006). Hydrogen production by Clostridiumthermocellum 27405 from cellulosic biomass substrates. International Journal of Hydrogen Energy 31, 1496-1503.

Liang, K., Zhang, Q., and Cong, W. (2012). Enzyme-Assisted Aqueous Extraction of Lipid from Microalgae. Journal of Agricultural and Food Chemistry, 60, 11771-11776.

Lin, P.P., Mi, L., Morioka, A.H., Yoshino, K.M., Konishi, S., Xu, S.C., Papanek, B.A., Riley, L.A., Guss, A.M., \& Liao, J.C. (2015). Consolidated bioprocessing of cellulose to isobutanol using Clostridium thermocellum. Metabolic engineering, 31, 44-52 .

Luque de Castro, M.D. and Garcia-Ayuso, L.E. (2000) Soxhlet Extraction of Solid Materials: An Outdated Technique with a Promising Innovative Future. Analytica Chimica Acta, 369,110.http://dx.doi.org/10.1016/S0003-2670(98)00233-5

Lynd, L.R., \& Grethlein, H.E. (1987). Hydrolysis of dilute acid pretreated mixed hardwood and purified microcrystalline cellulose by cell-free broth from Clostridium thermocellum. Biotechnology and bioengineering, 29 1, 92-100 .

Lynd, L. R., Van Zyl, W. H., McBride, J. E., \& Laser, M. (2005). Consolidated bioprocessing of cellulosic biomass: an update. Current opinion in biotechnology, 16(5), 577-583.

Mamidipally, P. K. and Liu, S. X. (2004), First approach on rice bran oil extraction using limonene. Eur. J. Lipid Sci. Technol., 106: 122-125. doi:10.1002/ej1t.200300891

Medipally, S. R., Yusoff, F. M., Banerjee, S., \& Shariff, M. (2015). Microalgae as sustainable renewable energy feedstock for biofuel production. BioMed Research International, $2015,1-13$.

Milledge, J.J. \& Heaven, S. (2013). A review of the harvesting of micro-algae for biofuel production Rev Environ Sci Biotechnol 12: 165. https://doi.org/10.1007/s11157-0129301-z 
Medina AR, Grima EM, Gimenez AG, Ibanez MJ. (1998). Downstream processing of algal polyunsaturated fatty acids. Biotechnology Advances 1998;16(3):517-80.

Mohr, A., \& Raman, S. (2015). Lessons from first generation biofuels and implications for the sustainability appraisal of second-generation biofuels. Efficiency and Sustainability in Biofuel Production, 63, 281-310.

Mohtasebi, B. (2013). Enhanced green production of biobutanol by novel fusants of two and three clostridia. (Unpublished Master's thesis, Ryerson University, Toronto, Canada). Retrieved from https://digital.library.ryerson.ca/islandora/object/RULA\%3A2179

Mood, S.H., Golfeshan, A.H., Tabatabaei, M., Abbasalizadeh, S., Ardjmand, M. \& SalehiJouzani, G.H. (2014).Comparison of different ionic liquids pretreatment for corn stover enzymatic saccharification. Preparative Biochemistry and Biotechnology 44(5): 451-463.

Motghare, K.A., Rathod, A.P.,Wasewar, K.L. \& Labhsetwar, N.K. 2016. Comparative study of different waste biomass for energy application. Waste Management 47: 40-45.

Mubarak, M. \& Shaija, A \& tv, Suchithra. (2014). A review on the extraction of lipid from microalgae for biodiesel production. Algal Research. 7. 10.1016/j.algal.2014.10.008.

Naghdi, F. G., González, L. M., Chan, W., \& Schenk, P. M. (2016). Progress on lipid extraction from wet algal biomass for biodiesel production. Microbial Biotechnology, 9(6), 718726. doi:10.1111/1751-7915.12360

Nakayama, S., Kiyoshi, K., Kadokura, T., and Nakazato, A. (2011). Butanol Production from Crystalline Cellulose by Cocultured Clostridium thermocellum and Clostridium saccharoperbutylacetonicum N1-4. Appl. Environ. Microbiol. 77, 6470-6475.

Nanda, S., Dalai, A. K., \& Kozinski, J. A. (2014). Butanol and ethanol production from lignocellulosic feedstock: biomass pretreatment and bioconversion. Energy Science \& Engineering, 2(3), 138-148.

Natarajan, R., Ang, W. M., Chen, X., Voigtmann, M., \& Lau, R. (2014). Lipid releasing characteristics of microalgae species through continuous ultrasonication. Bioresource Technology,158, 7-11. doi:10.1016/j.biortech.2014.01.146

Nguyen, M. T., Choi, S. P., Lee, J., Lee, J. H., \& Sim, S. J. (2009). Hydrothermal acid pretreatment of Chlamydomonas reinhardtii biomass for ethanol production. Journal of microbiology andbiotechnology, 19(2), 161-166.

Ozkan, A., Kinney, K., Katz, L. E., \& Berberoglu, H. (2012). Reduction of water and energy 
requirement of algae cultivation using an algae biofilm photobioreactor. Bioresource Technology, 114, 542-548. https://doi.org/10.1016/j.biortech.2012.03.055

Ohgren K., Bengtsson O., Gorwa-Grauslund M.F., Galbe M., Hahn-Hagerdal B., \& Zacchi G. (2006). Simultaneous saccharification and co-fermentation of glucose and xylose in steam-pretreated corn stover at high fiber content with Saccharomyces cerevisiae TMB3400. Journal of Biotechnology, 30, 488-498.

Pachauri, R.K. and Meyer, L.A., (2014). Climate Change Synthesis Report: Contributions of Working Groups Contribution of Working Groups I, II and III to the Fifth Assessment Report of the Intergovernmental Panel on Climate Change. Geneva, Switzerland

Passos, F.G., Uggetti, E., Carrère, H., \& Ferrer, I.B. (2014). Pretreatment of microalgae to improve biogas production: a review. Bioresource technology, 172, 403-412 .

Patáková, P., Branská, B., Sedlár, K., Vasylkivska, M., Jurečková, K., Kolek, J., Koščová, P., \& Provazník, I. (2018). Acidogenesis, solventogenesis, metabolic stress response and life cycle changes in Clostridium beijerinckii NRRL B-598 at the transcriptomic level. Scientific Reports.

Patil, P.D., Dandamudi, K.P.R., Wang, J., Deng, Q., and Deng, S. (2018). Extraction of bio-oils from algae with supercritical carbon dioxide and cosolvents. The Journal of Supercritcal Fluids, 135(3), 60-68.

Pfromm, P.H. \& Amanor-Boadu, Vincent \& Nelson, Richard \& Vadlani, Praveen \& Madl, Ronald. (2010). Bio-butanol vs. bio-ethanol: A technical and economic assessment for corn and switchgrass fermented by yeast or Clostridium acetobutylicum. Biomass and Bioenergy. 34. 515-524. 10.1016/j.biombioe.2009.12.017.

Plevin, R. J., Jones, A. D., Torn, M. S., \& Gibbs, H. K. (2010). Greenhouse gas emissions from biofuels' indirect land use changes are uncertain but may be much greater than previously estimated. Environmental Science \& Technology, 44(21), 8015-8021.

Pragya, Namita, Pandey, Krishan, \& , P.K.Sahoo. (2013). A review on harvesting, oil extraction and biofuels production technologies from microalgae. Renewable and Sustainable Energy Reviews. 24. 159-171. 10.1016/j.rser.2013.03.034.

Prabakaran, P., \& Ravindran, A.D. (2011). A comparative study on effective cell disruption methods for lipid extraction from microalgae. Letters in applied microbiology, 53 2, 1504 .

Puri, M., Abraham, R. E., \& Barrow, C. J. (2012). Biofuel production: Prospects, challenges and 
feedstock in Australia. Renewable and Sustainable Energy Reviews, 16(8), 6022-6031.

Qing, Q., Yang, B., \& Wyman, C. E. (2010). Xylooligomers are strong inhibitors of cellulose hydrolysis by enzymes. Bioresource technology, 101(24), 9624-9630.

Roux, J.D., Lamotte, H., \& Achard, J. (2017). An Overview of Microalgae Lipid Extraction in a Biorefinery Framework.

Roy, D. L., \& Klein, K. (2012). The policy objectives of a biofuel industry in Canada: An assessment. Agriculture, 2(4), 436-451.

Roy, P. (2014). Mutagenesis of novel clostridial fusants for enhanced green biobutanol production using renewable and sustainable feedstock. (Unpublished Master's Thesis, Ryerson University, Toronto) Retrieved from https://digital.library.ryerson.ca/islandora/object/RULA\%3A3688 Canada.

Ruthu, Murthy, P., Rai, A., \& Bhaskar, N. (2012). Fermentative recovery of lipids and proteins from freshwater fish head waste with reference to antimicrobial and antioxidant properties of protein hydrolysate. Journal of Food Science And Technology, 51(9), 18841892.

Ryckebosch, E., Muylaert, K. \& Foubert, I. J Am Oil Chem Soc (2012) 89: 189. https://doiorg.ezproxy.lib.ryerson.ca/10.1007/s11746-011-1903-z

Salmén, L. \& Bergstrom, E. 2009. Cellulose structural arrangement in relation to spectral changes in tensile loading FTIR. Cellulose 16: 975-982.

Santiago, J.C., David, E.S., \& ValentinoM.J., G. (2016). Proximate composition profiling of the rice straw enriched with mycoprotein of fungal endophytes.

Sati, H., Mitra, M., Mishra, S., \& Baredar, P.V. (2019). Microalgal lipid extraction strategies for biodiesel production: A review.

Sierra, L. S., Dixon, C. K., \& Wilken, L. R. (2017). Enzymatic cell disruption of the microalgae Chlamydomonas reinhardtii for lipid and protein extraction. Algal Research, 25, 149159.

Singh, D. P., \& Trivedi, R. K. (2013). Acid and alkaline pretreatment or lignocellulosic biomass to produce ethanol as biofuel. International Journal of ChemTech Research, 5(2), 727734.

Singh, R. S., Pandey, A., \& Gnansounou, E. (2017). Biofuels: productions and future 
perspectives. Boca Raton, FL: Taylor and Francis Group.

Skorupskaitė, V., Makarevičienė, V., Sendžikienè, E., \& Gumbytè, M. (2019). Microalgae

Chlorella sp. cell disruption efficiency utilising ultrasonication and ultrahomogenisation methods. Journal of Applied Phycology, 1-6.

Stevulova, N., Cigasova, J., Estokova, A., Terpakova, E., Geffert, A., Kacik, F., Holub, M. (2014). Properties Characterization of Chemically Modified Hemp Hurds. Materials, 7(12), 8131-8150. doi:10.3390/ma7128131

Sovacool, B. \& Mukherjee, I. (2011). Conceptualizing and measuring energy security: A synthesized approach. Energy, 36(8). 5343-5355.

Stanbury, P. F., Whitaker, A., \& Hall, S. J. (2017). Principles of fermentation technology. Oxford, England: Butterworth-Heinemann.

Syed, K., \& Dahman, Y. (2015). Novel clostridial fusants in comparison with co-cultured counterpart species for enhanced production of biobutanol using green renewable and sustainable feedstock. Bioprocess and Biosystems Engineering, 38(11), 2249-2262.

Tanger, P., Field, J.L., Jahn, C.E., DeFoortb, M.W.\&Leach, J.E. 2013. Biomass for thermochemical conversion:targets and challenges. Frontiers in Plant Science 4: 1-20.

Tanzi, C.D., Vian, M.A., Ginies, C., Elmaataoui, M., \& Chemat, F. (2012). Terpenes as Green Solvents for Extraction of Oil from Microalgae. Molecules.

Thirmal, C., \& Dahman, Y. (2011). Comparisons of existing pretreatment, saccharification, and fermentation processes for butanol production from agricultural residues. The Canadian Journal of Chemical Engineering, 90(3), 745-761.

Tian, K. and Zhi, L. (2016). High-yielding, one-pot, and green production of biodiesel from waste grease using wet cells of a recombinant Escherichia coli strain as catalyst. Biochemical Engineering Journal, 115(1), 30-37.

Tian, L., Cervenka, N.D., Low, A.M., Olson, D.G., \& Lynd, L.R. (2018). A mutation in the AdhE alcohol dehydrogenase of Clostridium thermocellum increases tolerance to several primary alcohols, including isobutanol, n-butanol and ethanol. Scientific Reports.

United States Energy Information Administration. (2016). International Energy Outlook. Washington, United States.

Qureshi, N., Saha, B.C., Hector, R.E., Hughes, S.R., and Cotta, M.A. (2008). Biobutanol 
production from wheat straw by simultaneous saccharification and fermentation using Clostridium beijerinckii: Part I-Batch fermentation. Biomass and Bioenergy, 32, 168175.

Ververis, C., Georghiou, K., Danielidis, D., Hatzinikolaou, D., Santas, P., Santas, R., \& Corleti, V. (2007). Cellulose, hemicelluloses, lignin and ash content of some organic materials a nd their suitability for use as paper pulp supplements. Bioresource Technology, 98(2), 296-301.

Vogel, J. 2008. Unique aspects of the grass cell wall. Current Opinion in Plant Biology 11: 301307.doi:10.1016/j.pbi.2008.03.002.

Wang ZY, Cao GL, Jiang C, Song JZ, Zheng J, et al. (2013) Butanol Production from Wheat Straw by Combining Crude Enzymatic Hydrolysis and Anaerobic Fermentation Using Clostridium acetobutylicum ATCC824. Energy Fuels 27: 5900-5906.

Wang, Long \& Liu, Ying \& Pu, Fan \& Zhang, Wen \& Zhou, Zhongmu. (2018). Effect of Rotary Evaporator Water Bath Temperature on Recovery Rate of Phthalate Esters. Bulletin of Environmental Contamination and Toxicology. 101. 10.1007/s00128-018-2440-3.

World Bioenergy Association. (2017). World Bioenergy Association Global BioenergyStatistics. Stockholm, Sweden.

Yang, B., Dai, Z., Ding, S.Y. \&Wyman, C.E. (2011). Enzymatic hydrolysis of cellulosic biomass. Biofuels 2(4): 421-449.

Yasarer, M.W. L., Sinnathamby, S., and Sturm, S.M.B. (2016). Impacts of biofuel-based landuse change on water quality and sustainability in a Kansas watershed. Agricultural Waste Management, 175(2), 4-14.

Yeong, T. K., Jiao, K., Zeng, X., Lin, L., Pan, S., \& Danquah, M. K. (2018). Microalgae for biobutanol production - Technology evaluation and value proposition. Algal Research, 31, 367-376.

Yin, C.Y. 2011. Prediction of higher heating values of biomass from proximate and ultimate analyses. Fuel. 90(3): 1128-1132

Yokoyama, S. 2008. The Asian Biomass Handbook, A guide for Biomass Production and Utilization. The Japan Institute of Energy: 1-388.

Zhang, Y., Kong, X., Wang, Z., Sun, Y., Zhu, S., Li, L., \& Lv, P. (2018). Optimization of 
enzymatic hydrolysis for effective lipid extraction from microalgae Scenedesmus sp. Renewable Energy, 125, 1049-1057.

Zhang, T., Hu, H., Wu, Y., Zhuang, L., Xu, X., Wang, X., \& Dao, G. (2016). Promising solutions to solve the bottlenecks in the large-scale cultivation of microalgae for biomass/bioenergy production. Renewable and Sustainable Energy Reviews, 60, 16021614.

Zhao, SH., Ma TS, Zhang HB (2011) Butanol production from halophyte seepweed Suaeda salsa by simultaneous saccharification and fermentation. Asian J Chem 23: 5285-528.

Zhao, X., Liu, W., Deng, Y., \& Zhu, J. (2016). Lignocellulosic biomass-energized fuel cells: Cases of high-temperature conversion. New York, NY: Momentum Press.

Zuliani, A., Ivars, F., \& Luque, R. (2017). Advances in nanocatalysts design for biofuels production. ChemCatChem, 10, 1-15. 